\title{
Extensões de Polinômios e de Funções Analíticas em Espaços de Banach
}

Victor dos Santos Ronchim

\section{DiSSERTAÇÃO APRESENTADA}

$\mathrm{AO}$

Instituto DE MATEMÁtica e Estatística

DA

Universidade DE SÃo PaUlo

PARA

OBTENÇÃO DO TÍTULO

$\mathrm{DE}$

Mestre EM MATEMÁticA

Programa: Matemática

Orientador: Prof ${ }^{\mathrm{a}}$. Dr ${ }^{\mathrm{a}}$. Daniela Mariz Silva Vieira

Durante o desenvolvimento deste trabalho o autor recebeu auxílio financeiro do CNPq

São Paulo, fevereiro de 2016 
Extensões de Polinômios e de Funções Analíticas em Espaços de Banach

Esta é a versão original da dissertação/tese elaborada pelo candidato Victor dos Santos Ronchim, tal como submetida à Comissão Julgadora. 


\section{Extensões de Polinômios e de Funções Analíticas em Espaços de Banach}

Esta versão da dissertação contém as correções e alterações sugeridas pela Comissão Julgadora durante a defesa da versão original do trabalho, realizada em 10/03/2017. Uma cópia da versão original está disponível no Instituto de Matemática e Estatística da Universidade de São Paulo.

Comissão Julgadora:

- Prof ${ }^{a}$. Dra ${ }^{\mathrm{a}}$. Daniela Mariz Silva Vieira (orientadora) - IME-USP

- Prof. Dr. Geraldo Marcio De Azevedo Botelho - UFU

- Prof. Dr. Vinícius Vieira Fávaro - UFU 
"A mathematician is a person who can find analogies between theorems; a better mathematician is one who can see analogies between proofs and the best mathematician can notice analogies between theories. One can imagine that the ultimate mathematician is one who can see analogies between analogies."

- Stefan Banach 


\section{Agradecimentos}

Agradeço à minha família por todo o amor e carinho. Em especial à minha mãe, por sempre investir na minha educação, por tudo o que fez e continua fazendo por mim. Ao meu padrastro, por todo o carinho e fé que sempre teve por mim.

Agradeço ao meu professor, Anderson, que sempre foi exemplo de paciência e de dedicação ao trabalho. E por despertar em mim, de uma maneira nada convencional, o desejo de estudar matemática.

Gostaria de dedicar este parágrafo à minha orientadora, Daniela, com quem tive a oportunidade de aprender desde a graduação. Por me aceitar como aluno, pela dedicação em me orientar, pelo tema sugerido e por toda ajuda que sempre me prestou durante estes anos.

Agradeço aos professores que tive durante a graduação e o mestrado, com os quais aprendi muito e que sempre me inspiraram a estudar mais. Barbara, Cordaro, Leonardo, Daniela, Pádua, Raul, Tausk, Zara. Em especial à Lilian, por tudo o que me ensinou e pela confiança que sempre demonstrou por mim.

Quero agradecer a todos os meus amigos do instituto, por toda ajuda que sempre me prestaram, todo incentivo que me deram, pelos fins de semana de estudos e por todas as risadas. Allan, Antonio, Bruno, Felipe, Fernando, Henrique, Ivo, Jonas, Kaique, Matheus, Matheus, Rodolfo, Rodrigo e Vinícius.

Agradeço também aos meus amigos de fora do instituto, pelos momentos de lazer nas horas de folga e pela compreensão que sempre tiveram comigo. Em especial ao Eduardo, por tudo o que me ensinou.

Agradeço ao CNPq pelo apoio financeiro. 


\section{Resumo}

RONCHIM, V. S. Extensões de Polinômios e de Funções Analíticas em Espaços de Banach. 2016. 111 f. Dissertação (Mestrado) - Instituto de Matemática e Estatística, Universidade de São Paulo, São Paulo, 2016.

Este trabalho tem como principal objetivo estudar extensões de aplicações multilineares, de polinômios homogêneos e de funções analíticas entre espaços de Banach. Desta maneira, nos baseamos em importantes trabalhos sobre o assunto. Inicialmente apresentamos o produto de Arens para álgebras de Banach, extensões de Aron-Berner e de Davie-Gamelin para aplicações multilineares e provamos que todas estas extensões coincidem. A partir destes resultados, apresentamos a extensão de polinômios homogêneos e o Teorema de Davie-Gamelin que afirma que, assim como no caso de aplicações multilineares, as extensões de polinômios preservam a norma e, como consequência deste teorema, apresentamos uma generalização do Teorema de Goldstine. Em seguida estudamos espaços de Banach regulares e simetricamente regulares, que são propriedades relacionadas com a unicidade de extensão e são definidas a partir do ideal de operadores lineares fracamente compactos $K^{w}(E, F)$. Finalmente apresentamos a extensão de uma função de $\mathscr{H}_{b}(E)$ para $\mathscr{H}_{b}\left(E^{\prime \prime}\right)$ e o resultado, de Ignacio Zalduendo, que caracteriza esta extensão em termos da continuidade fraca-estrela do operador diferencial de primeira ordem.

Palavras-chave: aplicações multilineares, polinômios homogêneos, funções holomorfas, extensões. 


\section{Abstract}

RONCHIM, V. S. Extensions of Polynomials and Analytic Functions on Banach Spaces. 2016. 120 f. Dissertação (Mestrado) - Instituto de Matemática e Estatística, Universidade de São Paulo, São Paulo, 2016.

The main purpose of this work is to study extensions of multilinear mappings, homogeneous polynomials and analytic functions between Banach Spaces. In this way, we rely on important works on the subject. Firstly we present the Arens-product for Banach algebras, the Aron-Berner and Davie-Gamelin extensions for multilinear mappings and we prove that all these extensions are the same. From these results, we present an extension for homogeneous polynomials and the Davie-Gamelin theorem which asserts that, as in the case of multilinear mappings, the polynomial extension is norm-preserving and, as a consequence of this theorem, we present a generalization of the Goldstine theorem. After that we study regular and symmetrically regular Banach spaces which are properties related to the uniqueness of the extension and are defined in the setting of weakly compact linear operators $K^{w}(E, F)$. Lastly, we present the extension of a function of $\mathscr{H}_{b}(E)$ to one in $\mathscr{H}_{b}\left(E^{\prime \prime}\right)$ and the result, according to Ignacio Zalduendo, which characterizes this extension in terms of weak-star continuity of the first order differential operator.

Keywords: multilinear mappings, homogeneous polynomials, holomorphic functions, extensions. 


\section{Sumário}

Lista de Símbolos $\quad$ xi

1 Introdução 1

1.1 Considerações Preliminares . . . . . . . . . . . . . . . . . 1

1.2 Organização do Trabalho . . . . . . . . . . . . . . . . . . . . . 2

2 Conceitos Preliminares $\quad 3$

2.1 Teoremas Fundamentais . . . . . . . . . . . . . . . . . . . . . 3

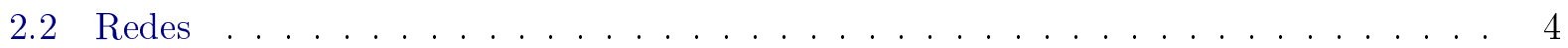

2.3 Topologia Induzida por uma Família de Funções . . . . . . . . . . . . . . . . . . . . 5

3 Polinômios em Espaços de Banach $\quad 9$

3.1 Aplicações Multilineares . . . . . . . . . . . . . . . . . . . . . . 9

3.2 Multilineares Simétricas e Multilineares Alternadas . . . . . . . . . . . . . . . 14

3.3 Polinômios e Polinômios Homogêneos . . . . . . . . . . . . . . . . . . . . . . 22

4 Extensões de Polinômios em Espaços de Banach $\quad 29$

4.1 Extensão de Operadores Lineares . . . . . . . . . . . . . . . . . . . . . . . . 29

4.2 Extensões de Arens . . . . . . . . . . . . . . . . . . . . . . . . . . . 31

4.3 Construção de Aron-Berner . . . . . . . . . . . . . . . . . . . . . . . . . . 40

4.4 Construção de Davie-Gamelin . . . . . . . . . . . . . . . . . . . . . . . . 46

4.5 Extensões de Polinômios Homogêneos . . . . . . . . . . . . . . . . . . . . . . . 47

5 Espaços Regulares e Simetricamente Regulares $\quad 53$

5.1 Operadores Fracamente Compactos . . . . . . . . . . . . . . . . . . 53

5.2 Produto de Arens . . . . . . . . . . . . . . . . . . . . . . . . . 57

5.3 Regularidade em Espaços de Banach . . . . . . . . . . . . . . . . . . . 63

6 Funções Holomorfas em Espaços de Banach $\quad 69$

6.1 Séries de Potências . . . . . . . . . . . . . . . . . . . . . . 69

6.2 Holomorfia em Espaços de Banach . . . . . . . . . . . . . . . . . . . . 73 
7 Extensão de Funções Holomorfas

7.1 O Espaço $\boldsymbol{G}_{\boldsymbol{E} \boldsymbol{F}}$ e a Extensão de Aron-Berner . . . . . . . . . . . . . . . . . 79

7.2 Extensão de funções holomorfas de tipo limitado . . . . . . . . . . . . . . . . 84

7.3 Extensão de funções holomorfas arbitrárias . . . . . . . . . . . . . . . . 89

7.4 Caracterização da extensão de funções holomorfas . . . . . . . . . . . . . . . . . . . 94 


\section{Lista de Símbolos}

$\mathbb{N} \quad$ o conjunto dos números inteiros estritamente positivos

$\mathbb{N}_{0} \quad \mathbb{N} \cup\{0\}$

$\mathbb{K} \quad$ os corpos $\mathbb{R}$ ou $\mathbb{C}$

$E, F \quad$ espaços de Banach sobre um corpo $\mathbb{K}$

$B_{E} \quad$ a bola unitária fechada no espaço normado $E$

$B(x, r) \quad$ a bola aberta de centro $x$ e raio $r>0$

$S_{E} \quad$ a esfera unitária no espaço normado $E$

$\bar{S} \quad$ fecho de um subconjunto $S \subset E$

$\partial U \quad$ fronteira de um subconjunto $U \subset E$

$l_{p}$

$l_{\infty}$

$X, Y$

$\mathscr{C}(K)$

$E^{\prime}$

$J_{E}$

o espaço de Banach $\left\{x=\left(x_{n}\right)_{n \in \mathbb{N}} \subset \mathbb{K}: \sum_{n=1}^{\infty}\left|x_{n}\right|^{p}<\infty\right\}$, onde $1 \leq p<\infty$

o espaço de Banach $\left\{x=\left(x_{n}\right)_{n \in \mathbb{N}} \subset \mathbb{K}: \sup _{n \in \mathbb{N}}\left|x_{n}\right|<\infty\right\}$

álgebras de Banach sobre $\mathbb{K}$

espaço das funções contínuas definidas em um espaço compacto Hausdorff a valores em $\mathbb{K}$

o espaço vetorial normado dos funcionais lineares e contínuos em $E$

$\mathcal{T}_{\|\cdot\|}$

imersão isométrica de $E$ em $E^{\prime \prime}$

$w$

a topologia gerada pela norma em $E$

$w^{*}$

a topologia fraca em $E$

$\mathscr{L}(E, F) \quad$ espaço normado dos operadores lineares e contínuos $T: E \longrightarrow F$

$\mathscr{K}(E, F) \quad$ espaço normado dos operadores lineares compactos $T: E \longrightarrow F$

$\mathscr{K}^{w}(E, F) \quad$ espaço normado dos operadores lineares fracamente compactos $T: E \longrightarrow F$

$G_{E F} \quad \mathscr{L}(\mathscr{L}(E, F), F)$

$J_{E, G_{E F}} \quad$ a imersão isométrica de $E$ em $G_{E F}$

$\mathscr{L}_{a}\left({ }^{m} E, F\right) \quad$ o espaço normado de todas as aplicações $m$-lineares $A: E^{m} \longrightarrow F$

$\mathscr{L}\left({ }^{m} E, F\right) \quad$ o subespaço vetorial de $\mathscr{L}_{a}\left({ }^{m} E, F\right)$ das aplicações $m$-lineares contínuas

$\mathscr{L}_{a}^{s}\left({ }^{m} E, F\right)$ o subespaço vetorial de $\mathscr{L}_{a}\left({ }^{m} E, F\right)$ das aplicações $m$-lineares simétricas

$\mathscr{L}^{s}\left({ }^{m} E, F\right) \quad \mathscr{L}_{a}^{s}\left({ }^{m} E, F\right) \cap \mathscr{L}\left({ }^{m} E, F\right)$

$\mathscr{L}_{a}^{a}\left({ }^{m} E, F\right)$ o subespaço vetorial de $\mathscr{L}_{a}\left({ }^{m} E, F\right)$ das aplicações $m$-lineares alternadas 
$\mathscr{L}^{a}\left({ }^{m} E, F\right) \quad \mathscr{L}_{a}^{a}\left({ }^{m} E, F\right) \cap \mathscr{L}\left({ }^{m} E, F\right)$

$\mathscr{P}_{a}\left({ }^{m} E, F\right) \quad$ o espaço vetorial de todos os polinômios $m$-homogêneos de $E$ em $F$

$\mathscr{P}\left({ }^{m} E, F\right) \quad$ o subespaço de $\mathscr{P}_{a}\left({ }^{m} E, F\right)$ formado pelos polinômios $m$-homogêneos contínuos

$\mathscr{P}_{a}(E, F) \quad$ o espaço vetorial de todos os polinômios de $E$ em $F$

$\mathscr{P}(E, F) \quad$ o subespaço de $\mathscr{P}_{a}(E, F)$ formado pelos polinômios contínuos

$\mathscr{H}(U, F) \quad$ o espaço vetorial das funções holomorfas $f: U \subset E \longrightarrow F$

$\mathscr{H}_{b}(U, F) \quad$ o espaço vetorial das funções holomorfas $f: U \subset E \longrightarrow F$ de tipo limitado

$R_{b} f(a) \quad$ o raio de limitação de uma função holomorfa $f$ no ponto $a$

$R_{c} f(a) \quad$ o raio de convergência (uniforme) da série de Taylor de $f$ em $a$

$D^{k} f(a) \quad$ diferencial de ordem $k$ de $f$ no ponto $a$ 


\section{Capítulo 1}

\section{Introdução}

\subsection{Considerações Preliminares}

O objetivo deste trabalho é estudar extensões de aplicações multilineares, de polinômios homogêneos e de funções holomorfas entre espaços de Banach.

O Teorema de Hahn-Banach [Meg12] afirma que todo funcional linear contínuo definido em um subespaço de um espaço de Banach pode ser estendido para todo o espaço, preservando norma. O problema de estender polinômios homogêneos de um subespaço de um espaço de Banach para todo o espaço foi estudado pela primeira vez por R. Aron e P. Berner em [AB78] em 1978. Eles mostraram que, em contraste com o caso de funcionais lineares, extensões de polinômios nem sempre existem. No entanto, demonstraram que para todo $n$, cada polinômio $n$-homogêneo em um espaço de Banach $E$ estende-se a um polinômio $n$-homogêneo em seu bidual $E^{\prime \prime}$. Este trabalho tornou-se bastante influente, muitos autores trataram de generalizá-lo para outras classes de aplicações não lineares [GGM93, GGMM94], bem como estudar as propriedades das extensões dos polômios homogêneos [DG89, ABC01]. Por exemplo, Davie e Gamelin, em [DG89], mostraram que a extensão de Aron-Berner [AB78] preserva a norma, além de possuir uma importante propriedade relacionada a continuidade fraca-estrela. A unicidade da extensão de Aron-Berner também é estudada em [ABC01]. Existem também artigos que tratam do assunto com mais detalhes, como em [SGD00, Za105, LZ00], além de propor versões alternativas e equivalentes que foram obtidas para as extensões.

A extensão de Aron-Berner representa um ponto importante no desenvolvimento da teoria de holomorfia em dimensão infinita, sobretudo no problema de estender aplicações holomorfas em espaços de Banach [Za190]. Temas como regularidade e operadores de Nicodemi também estão relacionados com a extensão de Aron-Berner para aplicações multilineares, o que dá grande importância a este assunto. 


\subsection{Organização do Trabalho}

A seguir descrevemos os assuntos abordados em cada capítulo. Os principais resultados deste trabalho encontram-se nos artigos [DG89], [Za105], [Za190], [Din12] e [Dan13].

No capítulo 1 apresentamos algumas definições e resultados referentes à Análise Funcional que serão utilizados ao longo deste trabalho. Os resultados deste capítulo constam em [Meg12].

O capítulo 2 apresenta resultados básicos da teoria de aplicações multilineares e de polinômios entre espaços de Banach. Utilizaremos estes objetos em todos os demais capítulos deste trabalho e, por este motivo, tratamos de relacionar os resultados aqui obtidos com àqueles conhecidos para operadores lineares. Os tópicos apresentados podem ser encontrados em [Muj86] e [Cha85].

No capítulo 3 apresentamos as extensões de aplicações multilineares entre espaços de Banach. Inicialmente relembramos como obter a extensão de um operador linear, dada pelo seu segundo adjunto. Em seguida apresentamos as extensões de Arens de uma aplicação bilinear contínua em $\mathscr{L}\left(E_{1}, E_{2} ; F\right)$. Posteriormente nos restringimos a aplicações multilineares com valores no corpo, apresentamos as extensões de Aron-Berner e Davie-Gamelin e provamos que estas extensões coincidem. Por fim, definimos a extensão de um polinômio homogêneo e provamos o Teorema de DavieGamelin, bem como uma generalização para o Teorema de Goldstine. Os resultados aqui apresentados constam em [DG89] e em [Dan13].

No capítulo 4 apresentamos duas importantes classes de espaços de Banach. Na primeira seção estudamos os resultados básicos sobre o ideal de operadores lineares fracamente compactos $\mathscr{K}^{w}(E, F)$. Na segunda seção estudamos álgebras de Banach e neste contexto, utilizamos a extensão de Arens para estender o produto de uma álgebra de Banach $X$ para o seu bidual de modo que $X^{\prime \prime}$ admita uma estrutura de álgebra de Banach. Apresentamos um exemplo em que se perde a comutatividade da álgebra neste processo, o que é equivalente a perder simetria no processo de extensão de uma aplicação bilinear contínua. Por fim apresentamos resultados básicos sobre regularidade de espaços de Banach, como em [Za105].

O capítulo 5 é dedicado ao estudo de holomorfia em espaços de Banach. Na primeira seção apresentamos resultados sobre séries de potências. Na seção seguinte apresentamos sucintamente os resultados fundamentais sobre holomorfia em espaços de Banach. Os tópicos apresentados neste capítulo se encontram em [Muj86], [Cha85] e [Nac69].

No capítulo 6 estudamos extensões de funções holomorfas e um teorema de caracterização para estas extensões. Na primeira seção apresentamos uma generalização da extensão de Aron-Berner. Na seção seguinte estudamos o caso de estender funções holomorfas de tipo limitado. Posteriormente apresentamos a extensão de funções holomorfas arbitrárias e, por último, apresentamos um teorema que caracteriza a extensão de funções em $\mathscr{H}_{b}(E)$ ao bidual em termos de continuidade fraca-estrela do operador diferencial de primeira ordem. Este capítulo é baseado no artigo [Zal90] e em [Din12]. 


\section{Capítulo 2}

\section{Conceitos Preliminares}

Neste capítulo apresentaremos os requisitos mínimos para a leitura deste trabalho. Boa parte das demonstrações pode ser encontrada em [Meg12].

Ao longo deste trabalho, a menos que se diga o contrário, $E$ e $F$ denotarão espaços de Banach sobre o mesmo corpo $\mathbb{K}$, onde $\mathbb{K}=\mathbb{R}$ ou $\mathbb{K}=\mathbb{C}$.

\section{$2.1 \quad$ Teoremas Fundamentais}

Nesta seção apresentaremos alguns dos resultados fundamentais de Análise Funcional que usaremos ao longo do trabalho.

Teorema 2.1.1 (Teorema de Hahn-Banach). Sejam E um espaço normado e $M$ um subespaço de E. Dado $\varphi \in M^{\prime}$, existe $\widetilde{\varphi} \in E^{\prime}$ tal que $\left.\widetilde{\varphi}\right|_{M}=\varphi$ e $\|\widetilde{\varphi}\|=\|\varphi\|$.

Corolário 2.1.2. Se x é um elemento não nulo de um espaço normado $E$, então existe $\varphi \in S_{E^{\prime}}$ tal que $\varphi(x)=\|x\|$.

Corolário 2.1.3. Seja $E \neq\{0\}$ um espaço normado. Para cada $x \in E$ :

$$
\|x\|=\max \left\{|\varphi(x)|: \varphi \in S_{E^{\prime}}\right\} .
$$

Teorema 2.1.4 (Teorema de Banach-Steinhaus). Sejam E um espaço de Banach, F um espaço normado e $\left\{T_{i}: i \in I\right\} \subset \mathscr{L}(E, F)$ tal que:

$$
\sup _{i \in I}\left\|T_{i}(x)\right\|<\infty \quad \text { para cada } x \in E .
$$

Então $\sup _{i \in I}\left\|T_{i}\right\|<\infty$.

O Teorema de Banach-Steinhaus nos diz que uma família de operadores é limitada se, e somente se, for pontualmente limitada. Por este motivo, este resultado também é conhecido por Princípio da Limitação Uniforme. 
O próximo resultado nos diz que todo operador linear, contínuo e sobrejetor entre espaços de Banach é uma aplicação aberta.

Teorema 2.1.5 (Teorema da Aplicação Aberta). Sejam E e F espaços de Banach e seja $T \in$ $\mathscr{L}(E, F)$. São equivalentes:

(a) T é sobrejetora;

(b) Existe $\delta>0$ tal que $\delta B_{F} \subset \overline{T\left(B_{E}\right)}$;

(c) Existe $\delta>0$ tal que $\delta B_{F} \subset T\left(B_{E}\right)$;

(d) T é aberta.

Além disso, todo operador linear contínuo e bijetor entre espaços de Banach é um isomorfismo topológico.

\subsection{Redes}

Definição 2.2.1. Um conjunto dirigido é um conjunto $I \neq \emptyset$ munido de uma relação $\preceq$ que satisfaz:

(a) $\alpha \preceq \alpha$ para todo $\alpha \in I$.

(b) Se $\alpha \preceq \beta$ e $\beta \preceq \gamma$, então $\alpha \preceq \gamma$.

(c) Para cada par de elementos $\alpha, \beta \in I$, existe $\gamma_{\alpha, \beta} \in I$ tal que $\alpha \preceq \gamma_{\alpha, \beta}$ e $\beta \preceq \gamma_{\alpha, \beta}$.

Isto é, um conjunto dirigido é um conjunto pré-ordenado que satisfaz a condição (c). Uma rede em um conjunto $\mathscr{X}$ é uma função $f: I \longrightarrow \mathscr{X}$, onde $I$ é um conjunto dirigido.

Analogamente ao caso das sequências, se $f: I \longrightarrow \mathscr{X}$ é uma rede em $\mathscr{X}$, denotamos $f(\alpha)=x_{\alpha}$ e escrevemos $f=\left(x_{\alpha}\right)_{\alpha \in I}$ e, em algumas situações, escreveremos $\left(x_{\alpha}\right)$ para simplificar a escrita.

Definição 2.2.2. Dizemos que uma rede $\left(x_{\alpha}\right)_{\alpha \in I}$ em um espaço topológico $\mathscr{X}$ converge para $x \in \mathscr{X}$ se, para cada vizinhança aberta $U$ de $x$, existe $\alpha_{0} \in I$ tal que $x_{\alpha} \in U$ sempre que $\alpha \succeq \alpha_{0}$.

A convergência é denotada por $x_{\alpha} \longrightarrow x$ ou, caso haja unicidade do limite, $x=\lim _{\alpha} x_{\alpha}$.

Utilizamos sequências para caracterizar a topologia de um espaço métrico. O conceito de convergência de rede estende muitos destes resultados para espaços topológicos, como veremos a seguir:

Proposição 2.2.3. Sejam $S$ um subconjunto de um espaço topológico $\mathscr{X}$ e $x \in \mathscr{X}$. Então $x \in \bar{S}$ se, e somente se, existe uma rede $\left(x_{\alpha}\right) \subset S$ tal que $x_{\alpha} \longrightarrow x$.

Proposição 2.2.4. Sejam $\mathscr{X}$ e $\mathscr{Y}$ espaços topológicos. Uma função $f: \mathscr{X} \longrightarrow \mathscr{Y}$ é contínua em $x \in \mathscr{X}$ se, e somente se, $f\left(x_{\alpha}\right) \longrightarrow f(x)$ para toda rede $\left(x_{\alpha}\right) \subset \mathscr{X}$ convergente à $x$. 
A seguir vamos definir o conceito de subrede. Para isso precisamos da idéia de subconjuntos arbitrariamente grandes de um conjunto dirigido.

Definição 2.2.5. Um subconjunto $\Lambda$ de um conjunto dirigido $I$ é dito cofinal em $I$ se, para cada $\alpha \in I$, existe $\beta_{\alpha} \in \Lambda$ tal que $\alpha \preceq \beta_{\alpha}$.

Sejam $\mathscr{X}$ um conjunto, $I$ um conjunto dirigido e $f: I \longrightarrow \mathscr{X}$ uma rede em $\mathscr{X}, J$ um conjunto dirigido e $g: J \longrightarrow I$ uma função que satisfaz:

(a) $g\left(\beta_{1}\right) \preceq g\left(\beta_{2}\right)$ em I sempre que $\beta_{1} \preceq \beta_{2}$ em $J$.

(b) $g(J)$ é cofinal em $I$.

Então a rede $f \circ g: J \longrightarrow \mathscr{X}$ é dita uma subrede de $f$.

Note que, com a definição acima, uma subrede de uma sequência não é necessariamente uma subsequência. A seguir, destacamos algumas propriedades de subredes.

Proposição 2.2.6. Seja $\left(x_{\alpha}\right)$ uma rede em um espaço topológico $\mathscr{X}$. Então:

(a) Toda subrede de $\left(x_{\alpha}\right)$ é uma rede em $\mathscr{X}$.

(b) Toda subrede de uma subrede de $\left(x_{\alpha}\right)$ é uma subrede de $\left(x_{\alpha}\right)$.

(c) Se $x_{\alpha} \longrightarrow x$, então toda subrede de $\left(x_{\alpha}\right)$ converge à $x$.

\subsection{Topologia Induzida por uma Família de Funções}

Aqui apresentaremos como construir uma topologia induzida por uma família de funções, bem como fixaremos a notação para as topologias fraca e fraca* em espaços de Banach.

Definição 2.3.1. Sejam $\mathscr{X}$ um conjunto não vazio, $\mathscr{F}$ uma familia de funções tal que cada $f \in \mathscr{F}$ leva $\mathscr{X}$ em um espaço topológico $\left(\mathscr{Y}_{f}, \mathcal{T}_{\mathscr{Y}_{f}}\right)$. Definimos a topologia $\sigma(\mathscr{X}, \mathscr{F})$ como sendo a menor topologia em $\mathscr{X}$ que torna todos os elementos de $\mathscr{F}$ contínuos.

Dizemos que $\sigma(\mathscr{X}, \mathscr{F})$ é a topologia induzida por $\mathscr{F}$ em $\mathscr{X}$.

Exemplo 2.3.2. Sejam $(\mathscr{Y}, \mathcal{T})$ um espaço topológico e $\mathscr{X} \subset \mathscr{Y}$ com a topologia usual. Se $i: \mathscr{X} \hookrightarrow$ $\mathscr{Y}$ é a inclusão, considerando a família $\mathscr{F}=\{i\}$, segue que $\sigma(\mathscr{X},\{i\})=\{V \cap \mathscr{X}: V \in \mathcal{T}\}$.

Proposição 2.3.3. Sejam $\mathscr{X}$ e $\mathscr{F}$ como na definição anterior. Seja $\mathscr{B}$ o conjunto de todos os subconjuntos de $\mathscr{X}$ da forma:

$$
\bigcap_{i=1}^{n} f_{i}^{-1}\left(V_{i}\right), \quad \text { onde } n \in \mathbb{N}, f_{i} \in \mathscr{F}, V_{i} \in \mathcal{T}_{\mathscr{Y}_{f_{i}}} \text { para cada } 1 \leq i \leq n
$$

Então $\mathscr{B}$ é uma base de abertos para a topologia $\sigma(\mathscr{X}, \mathscr{F})$. 
O próximo resultado nos dá uma caracterização para convergência de redes na topologia induzida por uma família de funções.

Proposição 2.3.4. Uma rede $\left(x_{\alpha}\right) \subset \mathscr{X}$ converge para $x$ na topologia $\sigma(\mathscr{X}, \mathscr{F})$ se, e somente se, $f\left(x_{\alpha}\right) \longrightarrow f(x)$ para cada $f \in \mathscr{F}$.

Se $E$ é um espaço normado, o espaço $E^{\prime}$ é uma família de funções. O que nos leva à próxima definição.

Definição 2.3.5. Seja E é um espaço normado. A topologia $w=\sigma\left(E, E^{\prime}\right)$ é dita a topologia fraca de $E$.

Denotaremos por $\mathcal{T}_{\|\cdot\|}$ a topologia gerada pela norma em $E$. Pelo que acabamos de definir, segue que $\sigma\left(E, E^{\prime}\right) \leq \mathcal{T}_{\|\cdot\|}$. Além disso, dados $x \in E, \varphi_{1}, \ldots, \varphi_{n} \in E^{\prime}$ e $\varepsilon>0$, os conjuntos da forma:

$$
V\left(x ; \varphi_{1}, \ldots, \varphi_{n} ; \varepsilon\right) \doteq\left\{y \in E:\left|\varphi_{i}(x)-\varphi_{i}(y)\right|<\varepsilon, 1 \leq i \leq m\right\}=\bigcap_{i=1}^{n} \varphi_{i}^{-1}\left(B\left(\varphi_{i}(x), \varepsilon\right)\right.
$$

formam uma base para a topologia fraca de $E$.

E ainda, a convergência de redes na topologia fraca é caracterizada por:

$$
x_{\alpha} \stackrel{w}{\longrightarrow} x \Longleftrightarrow \varphi\left(x_{\alpha}\right) \longrightarrow \varphi(x) \text { para cada } \varphi \in E^{\prime} .
$$

Se $E$ é normado, podemos considerar a inclusão canônica de $E$ em seu bidual $E^{\prime \prime}, J_{E}: E \longrightarrow E^{\prime \prime}$, dada por $J_{E}(x)(\varphi) \doteq \varphi(x)$. A inclusão canônica é uma imersão isométrica e, por este motivo, identificamos $E=J_{E}(E) \subset E^{\prime \prime}$.

Definição 2.3.6. Sejam $E$ um espaço normado e $E^{\prime}$ o seu dual. A topologia $w^{*}=\sigma\left(E^{\prime}, J_{E}(E)\right)=$ $\sigma\left(E^{\prime}, E\right)$ é dita a topologia fraca-estrela de $E^{\prime}$.

Analogamente ao que fizemos para a topologia fraca, dados $\varphi \in E^{\prime}, x_{1}, \ldots, x_{n} \in E$ e $\varepsilon>0$, os conjuntos da forma:

$$
V\left(\varphi ; x_{1}, \ldots, x_{n} ; \varepsilon\right)=\left\{\psi \in E^{\prime}:\left|\varphi\left(x_{i}\right)-\psi\left(x_{i}\right)\right|<\varepsilon, 1 \leq i \leq m\right\}
$$

formam uma base de abertos para a topologia fraca-estrela de $E^{\prime}$.

Podemos também considerar a topologia fraca de $E^{\prime}, \sigma\left(E^{\prime}, E^{\prime \prime}\right)$, e vale que $\sigma\left(E^{\prime}, E\right) \leq \sigma\left(E^{\prime}, E^{\prime \prime}\right) \leq$ $\mathcal{T}_{\|\cdot\|}$. Além disso, a convergência de redes na topologia fraca-estrela é caracterizada por:

$$
\begin{aligned}
\varphi_{\alpha} \stackrel{w^{*}}{\longrightarrow} \varphi & \Longleftrightarrow J_{E}(x)\left(\varphi_{\alpha}\right) \longrightarrow J_{E}(x)(\varphi) \text { para cada } x \in E \\
& \Longleftrightarrow \varphi_{\alpha}(x) \longrightarrow \varphi(x) \text { para cada } x \in E .
\end{aligned}
$$


Ao longo deste trabalho sempre explicitaremos as topologias envolvidas em conceitos de convergência, continuidade de funções e compacidade como indicamos a seguir:

$\operatorname{Sejam~}\left(X, \mathcal{T}_{1}\right)$ e $\left(Y, \mathcal{T}_{2}\right)$ espaços topológicos.

(a) $x_{\alpha} \stackrel{\mathcal{T}_{1}}{\rightarrow} x$ indica que a rede $\left(x_{\alpha}\right)$ converge para $x \in X$ segundo a topologia $\mathcal{T}_{1}$;

(b) Um subconjunto $K \subset X$ é $\mathcal{T}_{1}$-compacto indica que é compacto segundo a topologia $\mathcal{T}_{1}$. Utilizaremos a mesma notação para qualquer outra propriedade topológica;

(c) Uma função $f: X \longrightarrow Y$ é $\mathcal{T}_{1}$ - $\mathcal{T}_{2}$-contínua se for contínua em relação as topologias $\mathcal{T}_{1}$ em $X$ e $\mathcal{T}_{2}$ em $Y$. No caso em que $X=Y$ e $\mathcal{T}_{1}=\mathcal{T}_{2}$, dizemos simplesmente que $f: X \longrightarrow X$ é $\mathcal{T}_{1}$-contínua.

A seguir enunciaremos alguns resultados importantes dessas topologias, que serão utilizados ao longo do trabalho.

Proposição 2.3.7. Sejam E e F espaços normados e $T: E \longrightarrow F$ linear. Então $T$ é $\|\cdot\|$-contínuo se, e somente se, $T$ é w-contínuo.

Proposição 2.3.8. Seja $E$ um espaço normado. A inclusão canônica $J_{E}: E \longrightarrow E^{\prime \prime}$ é um w-w*homeomorfismo sobre a sua imagem.

Definição 2.3.9. Sejam E e $F$ espaços de Banach e $T \in \mathscr{L}(E, F)$. O operador $T^{\prime}: F^{\prime} \longrightarrow E^{\prime}$, dado por $T^{\prime}(\varphi) \doteq \varphi \circ T$ é chamado de operador adjunto de $T$.

Proposição 2.3.10. Sejam E e $F$ espaços de Banach e $T \in \mathscr{L}(E, F)$. Então $T^{\prime} \in \mathscr{L}\left(F^{\prime}, E^{\prime}\right)$ é $w^{*}-w^{*}$-continuo.

Teorema 2.3.11 (Teorema de Mazur). Sejam E um espaço normado e A um subconjunto convexo de E. Então, $\bar{A}^{\|\cdot\|}=\bar{A}^{w}$.

Teorema 2.3.12 (Teorema de Goldstine). Seja E um espaço normado. Então:

(a) $B_{E^{\prime \prime}}={\overline{B_{E}}}^{w^{*}}$.

(b) $E^{\prime \prime}=\bar{E}^{w^{*}}$.

Teorema 2.3.13. Um espaço normado E é reflexivo se, e somente se, $B_{E}$ é w-compacta.

Teorema 2.3.14 (Teorema de Alaoglu). Seja E um espaço normado. Então $B_{E^{\prime}}$ é w*-compacta.

Corolário 2.3.15. Seja $E$ um espaço normado. Um subconjunto $S \subset E$ é w*-compacto se, e somente se, $S$ é limitado e $w^{*}$-fechado. 
Proposição 2.3.16. Seja A um subconjunto de um espaço normado E. São equivalentes:

(a) A é w-compacto.

(b) $J_{E}(A)$ é $w^{*}$-compacto.

(c) A é limitado e $J_{E}(A)$ é $w^{*}$-fechado.

Teorema 2.3.17 (Teorema de Eberlein-Smulian). Sejam E um espaço normado e $K$ um subconjunto de E. Então $K$ é fracamente compacto se, e somente se, é fracamente sequencialmente compacto. 


\section{Capítulo 3}

\section{Polinômios em Espaços de Banach}

Neste capítulo serão apresentados resultados básicos sobre aplicações multilineares entre espaços de Banach para que, posteriormente, possamos definir e estudar polinômios e polinômios $m$-homogêneos nestes espaços. Os resultados aqui apresentados são baseados em [Muj86] e [Cha85].

\subsection{Aplicações Multilineares}

Dados $m \in \mathbb{N}$ e $E$ um espaço de Banach, denotamos $E^{m} \doteq\left\{\left(x_{1}, \ldots, x_{m}\right): x_{j} \in E, \quad 1 \leq j \leq m\right\}$. Observe que $E^{m}$ é um espaço de Banach quando munido da norma $\left\|\left(x_{1}, \ldots, x_{m}\right)\right\| \doteq \sup _{1 \leq j \leq m}\left\|x_{j}\right\|$.

Definição 3.1.1. Para cada $m \in \mathbb{N}$ denotamos por $\mathscr{L}_{a}\left({ }^{m} E, F\right)$ o espaço vetorial de todas as aplicações m-lineares $A: E^{m} \longrightarrow F$, munido com as operações de soma e multiplicação por escalar definidas pontualmente. Denotaremos por $\mathscr{L}\left({ }^{m} E, F\right)$ o subespaço vetorial de $\mathscr{L}_{a}\left({ }^{m} E, F\right)$ formado por todas as aplicações m-lineares que são contínuas em relação às normas dos espaços $E^{m}$ e $F$.

Para simplificar a notação, quando $m=1$ escrevemos $\mathscr{L}_{a}\left({ }^{1} E, F\right)=\mathscr{L}_{a}(E, F)$ e $\mathscr{L}\left({ }^{1} E, F\right)=$ $\mathscr{L}(E, F)$. No caso em que $F=\mathbb{K}$, escrevemos $\mathscr{L}_{a}\left({ }^{m} E, F\right)=\mathscr{L}_{a}\left({ }^{m} E\right)$ e $\mathscr{L}\left({ }^{m} E, F\right)=\mathscr{L}\left({ }^{m} E\right)$. Finalmente, quando $m=1$ e $F=\mathbb{K}$, escrevemos $\mathscr{L}_{a}(E)=E^{*}$ e $\mathscr{L}(E)=E^{\prime}$.

Muitos resultados que são válidos para operadores lineares podem ser estendidos facilmente para aplicações multilineares. A seguir apresentamos uma caracterização para a continuidade de uma aplicação $m$-linear.

Teorema 3.1.2. Para cada $A \in \mathscr{L}\left({ }^{m} E, F\right)$, são equivalentes:

(a) A é contínuo;

(b) A é contínuo na origem;

(c) Existe $M>0$ tal que, para cada $\left(x_{1}, \ldots, x_{m}\right) \in E^{m},\left\|A\left(x_{1}, \ldots, x_{m}\right)\right\| \leq M\left\|x_{1}\right\| \cdots\left\|x_{m}\right\|$. 
Demonstração. (a) $\Longrightarrow$ (b): Imediato.

(b) $\Longrightarrow($ c): Pela continuidade na origem, existe um $\delta>0$ tal que $\|A(y)\| \leq 1$ sempre que $\|y\| \leq \delta$.

Dado $x=\left(x_{1}, \ldots, x_{m}\right)$ qualquer, a desigualdade em (c) é válida se algum $x_{i}=0$. Suponha que cada $x_{i} \neq 0$. Então o ponto $z \doteq \delta\left(\frac{x_{1}}{\left\|x_{1}\right\|}, \ldots, \frac{x_{m}}{\left\|x_{m}\right\|}\right)$ é tal que $\|A(z)\| \leq 1$. Como $A$ é multilinear:

$$
\left\|A\left(x_{1}, \ldots, x_{m}\right)\right\| \leq \frac{1}{\delta^{m}}\left\|x_{1}\right\| \cdots\left\|x_{m}\right\|
$$

(c) $\Longrightarrow(\mathrm{a}):$ Dados $a=\left(a_{1}, \ldots, a_{m}\right)$ e $x=\left(x_{1}, \ldots, x_{m}\right)$ em $E^{m}$, temos:

$$
\begin{aligned}
\|A(x)-A(a)\| \leq & \left\|A\left(x_{1}-a_{1}, x_{2}, \ldots, x_{m}\right)\right\|+\left\|A\left(a_{1}, x_{2}-a_{2}, x_{3}, \ldots, x_{m}\right)\right\| \\
& +\ldots+\left\|A\left(a_{1}, \ldots, a_{m-1}, x_{m}-a_{m}\right)\right\| \\
\leq & \sum_{j=1}^{m} M\left\|a_{1}\right\| \ldots\left\|a_{j-1}\right\|\left\|a_{j}-x_{j}\right\|\left\|x_{j+1}\right\| \ldots\left\|x_{m}\right\|
\end{aligned}
$$

Assim, se $\|x-a\| \leq 1$, segue que $\|A(x)-A(a)\| \leq m M(\|a\|+1)^{m-1}\|x-a\|$ e isso mostra que a aplicação $A$ é contínua.

Observação 3.1.3. No teorema anterior, diferentemente do resultado análogo para operadores lineares, não foi demonstrado que uma aplicação multilinear contínua é uniformemente contínua. Isto se deve ao fato de que a única aplicação multilinear uniformemente contínua é a aplicação nula:

Suponha que $A \in \mathscr{L}\left({ }^{m} E, F\right)$, com $m \geq 2$, é não nula. Tome $\left(x_{1}, \ldots, x_{m}\right) \in E^{m}$ tal que $\left\|A\left(x_{1}, \ldots, x_{m}\right)\right\|>0$. Então cada $x_{i}$ é não nulo. Se $\varepsilon<\left\|A\left(x_{1}, \ldots, x_{m}\right)\right\|$, para cada $\delta>0$ dado, tome $\lambda \in \mathbb{K}$ tal que $0<|\lambda|<\frac{\delta}{\left\|x_{1}\right\|}$. Assim:

$$
\left\|\left(x_{1}+\lambda x_{1}, \frac{x_{2}}{\lambda}, x_{3}, \ldots, x_{m}\right)-\left(x_{1}, \frac{x_{2}}{\lambda}, x_{3}, \ldots, x_{m}\right)\right\|<\delta .
$$

Por outro lado, $\left\|A\left(x_{1}+\lambda x_{1}, \frac{x_{2}}{\lambda}, x_{3}, \ldots, x_{m}\right)-A\left(x_{1}, \frac{x_{2}}{\lambda}, x_{3}, \ldots, x_{m}\right)\right\|>\varepsilon$. Logo, A não é uniformemente continua.

Entretanto, uma aplicação multilinear contínua é uniformemente contínua sobre os conjuntos limitados.

O teorema anterior nos mostra que, assim como no caso linear, as aplicações multilineares contínuas levam conjuntos limitados de $E^{m}$ em conjuntos limitados de $F$. Isto nos permite definir a seguinte função:

$$
\|\cdot\|: \mathscr{L}\left({ }^{m} E, F\right) \longrightarrow\left[0,+\infty\left[\text { dada por } \quad\|A\| \doteq \sup _{\|x\| \leq 1}\|A(x)\| .\right.\right.
$$


Segue também do Teorema 3.1.2 que uma multilinear $A$ é contínua se, e somente se, $\|A\|<\infty$. Além disso, esta é a menor constante que satisfaz a condição 3.1.2(c) :

Dado $\left(x_{1}, \ldots, x_{m}\right) \in E^{m}$, temos $\left\|A\left(\frac{x_{1}}{\left\|x_{1}\right\|}, \ldots, \frac{x_{m}}{\left\|x_{m}\right\|}\right)\right\| \leq\|A\|$, por multi-linearidade obtemos:

$$
\left\|A\left(x_{1}, \ldots, x_{m}\right)\right\| \leq\|A\|\left\|x_{1}\right\| \ldots\left\|x_{m}\right\|
$$

Isto é, $\|A\|$ satisfaz a condição 3.1.2(c). Por outro lado, se $M>0$ é uma constante que satisfaz 3.1.2(c), então dado $x \in B_{E^{m}}$, temos $\left\|A\left(x_{1}, \ldots, x_{m}\right)\right\| \leq M$. Assim segue que $\|A\| \leq M$ e podemos concluir que:

$$
\|A\|=\inf \left\{M>0:\left\|A\left(x_{1}, \ldots, x_{m}\right)\right\| \leq M\left\|x_{1}\right\| \cdots\left\|x_{m}\right\| \quad \forall\left(x_{1}, \ldots, x_{m}\right) \in E^{m}\right\}
$$

Teorema 3.1.4. Uma aplicação $A \in \mathscr{L}_{a}\left({ }^{m} E, F\right)$ é contínua se, e somente se, é separadamente continua em cada variável.

Demonstração. Suponha que $A$ seja contínua. Então para cada $1 \leq i \leq m$, a função coordenada dada por $x_{i} \stackrel{f_{i}}{\longmapsto} A\left(x_{1}, \ldots, x_{i}, \ldots, x_{m}\right)$ é a restrição $f_{i}=\left.A\right|_{\left\{x_{1}\right\} \times \ldots \times E \times \ldots\left\{x_{m}\right\}}$, logo é contínua.

Provaremos a recíproca por indução em $m$.

Se $A$ é bilinear separadamente contínua, então as aplicações $A_{x}(y) \doteq A(x, y)$ e $A_{y}(x) \doteq A(x, y)$ são lineares e contínuas. Logo existe $M_{x}>0$ tal que, para cada $y \in E$ :

$$
\|A(x, y)\|=\left\|A_{y}(x)\right\|=\left\|A_{x}(y)\right\| \leq M_{x}\|y\| .
$$

Note que a família $\mathcal{F} \doteq\left\{A_{y} \in \mathscr{L}(E, F): y \in B_{E}\right\}$ é pontualmente limitada. Pelo Teorema de Banach-Steinhaus, existe $M>0$ tal que $\sup _{y \in B_{E}}\left\|A_{y}\right\| \leq M$. Assim, dados para cada $x, y \in B_{E}$ :

$$
\|A(x, y)\|=\left\|A_{y}(x)\right\| \leq\left\|A_{y}\right\|\|x\| \leq M .
$$

Segue que $A$ é bilinear contínua.

Agora suponha que toda aplicação $(m-1)$-linear separadamente contínua em cada variável é contínua. Seja $A \in \mathscr{L}_{a}\left({ }^{m} E, F\right)$ separadamente contínua, vejamos que $A$ é contínua.

Para cada $x_{m} \in E$, a função $A_{x_{m}}\left(x_{1}, \ldots, x_{m-1}\right) \doteq A\left(x_{1}, \ldots, x_{m}\right)$ é $(m-1)$-linear e separadamente contínua, e pela hipótese de indução, é contínua. Existe um $M_{x_{m}}>0$ tal que:

$$
\left\|A\left(x_{1}, \ldots, x_{m}\right)\right\|=\left\|A_{x_{m}}\left(x_{1}, \ldots, x_{m-1}\right)\right\| \leq M_{x_{m}}\left\|x_{1}\right\| \ldots\left\|x_{m-1}\right\| .
$$

Note que a família $\mathcal{F} \doteq\left\{A_{x_{1}, \ldots, x_{m-1}} \in \mathscr{L}(E, F): x_{i} \in B_{E}, 1<i<m\right\}$ é pontualmente limitada e, pelo Teorema de Banach-Steinhaus, existe $M>0$ tal que $\sup _{\mathcal{F}}\left\|A_{x_{1}, \ldots, x_{m-1}}\right\| \leq M$. 
Se $x_{i} \in B_{E}$ para $1 \leq i \leq m$, então:

$$
\left\|A\left(x_{1}, \ldots, x_{m}\right)\right\|=\left\|A_{x_{1}, \ldots, x_{m-1}}\left(x_{m}\right)\right\| \leq M\left\|x_{m}\right\| \leq M .
$$

Segue que $A$ é $m$-linear contínua.

Corolário 3.1.5. Se E é um espaço normado de dimensão finita, então toda aplicação multilinear definida em $E^{m}$ é contínua.

Proposição 3.1.6. A aplicação $\|\cdot\|$ é uma norma em $\mathscr{L}\left({ }^{m} E, F\right)$.

Demonstração. Seja $A \in \mathscr{L}\left({ }^{m} E, F\right)$ tal que $\|A\|=0$. Então, para cada $\left(x_{1}, \ldots, x_{m}\right) \in E^{m}$, temos:

$$
\left\|A\left(x_{1}, \ldots, x_{m}\right)\right\| \leq\|A\|\left\|x_{1}\right\| \ldots\left\|x_{m}\right\|=0 .
$$

E segue que $A \equiv 0$.

Dados $\lambda \in \mathbb{K}$ e $A \in \mathscr{L}\left({ }^{m} E, F\right)$, temos:

$$
\|\lambda A\|=\sup _{x \in B_{E^{m}}}\|\lambda A(x)\|=|\lambda| \sup _{x \in B_{E^{m}}}\|A(x)\|=|\lambda|\|A\| .
$$

Dados $A_{1}, A_{2} \in \mathscr{L}\left({ }^{m} E, F\right)$, para cada $x \in B_{E^{m}}:$

$$
\left\|\left(A_{1}+A_{2}\right)(x)\right\| \leq\left\|A_{1}(x)\right\|+\left\|A_{2}(x)\right\| \leq\left\|A_{1}\right\|+\left\|A_{2}\right\| .
$$

Tomando o supremo sobre $B_{E^{m}}$, segue a desigualdade triangular.

Proposição 3.1.7. Se F é um espaço de Banach, então $\mathscr{L}\left({ }^{m} E, F\right)$ se torna um espaço de Banach com a norma $\|\cdot\|$.

Demonstração. Seja $\left(A_{n}\right)$ uma sequência de Cauchy em $\mathscr{L}\left({ }^{m} E, F\right)$. Então, dado $\varepsilon>0$, existe $n_{0} \in \mathbb{N}$ tal que:

$$
j, k>n_{0} \Longrightarrow\left\|A_{j}-A_{k}\right\|<\varepsilon .
$$

Assim, para cada $x=\left(x_{1}, \ldots, x_{m}\right) \in E^{m}$, temos:

$$
\left\|A_{j}\left(x_{1}, \ldots, x_{m}\right)-A_{k}\left(x_{1}, \ldots, x_{m}\right)\right\| \leq\left\|A_{j}-A_{k}\right\|\left\|x_{1}\right\| \ldots\left\|x_{m}\right\| .
$$

Isto é, $\left(A_{n}\left(x_{1}, \ldots, x_{m}\right)\right)$ é uma sequência de Cauchy em $F$ e, portanto, convergente. Deste modo podemos definir $A: E^{m} \longrightarrow F$ por: $A\left(x_{1}, \ldots, x_{m}\right)=\lim _{n \rightarrow \infty} A_{n}\left(x_{1}, \ldots, x_{m}\right)$ e, desta definição, é imediato que $A \in \mathscr{L}_{a}\left({ }^{m} E, F\right)$. 
Além disso, como a sequência $\left(A_{n}\right)$ é limitada, por ser sequência de Cauchy, então existe $M>0$ tal que $\left\|A_{n}\right\| \leq M$ para todo $n \in \mathbb{N}$. Se $x \in B_{E^{m}}$ :

$$
\|A(x)\|=\left\|\lim _{n \rightarrow \infty} A_{n}(x)\right\|=\lim _{n \rightarrow \infty}\left\|A_{n}(x)\right\| \leq \lim _{n \rightarrow \infty}\left\|A_{n}(x)\right\| \leq \lim _{n \rightarrow \infty}\left\|A_{n}\right\| \leq M .
$$

Ou seja, $A \in \mathscr{L}\left({ }^{m} E, F\right)$. Por último, fixado $j \geq n_{0}$, para cada $x \in B_{E^{m}}$ segue que:

$$
\left\|\left(A_{j}-A\right)(x)\right\|=\left\|A_{j}(x)-A(x)\right\|=\left\|A_{j}(x)-\lim _{n} A_{n}(x)\right\|=\lim _{n}\left\|A_{j}(x)-A_{n}(x)\right\| \leq \varepsilon .
$$

Tomando o supremo sobre $B_{E^{m}}$, mostramos que $\left\|A_{n}-A\right\| \longrightarrow 0$ e, portanto, $\mathscr{L}\left({ }^{m} E, F\right)$ é espaço de Banach.

Teorema 3.1.8. Sejam $m, n \in \mathbb{N}$. A função $\Psi: \mathscr{L}_{a}\left({ }^{m+n} E, F\right) \longrightarrow \mathscr{L}_{a}\left({ }^{m} E, \mathscr{L}_{a}\left({ }^{n} E, F\right)\right)$ dada por:

$$
\Psi(A)\left(x_{1}, \ldots, x_{m}\right)\left(y_{1}, \ldots, y_{n}\right) \doteq A\left(x_{1}, \ldots, x_{m}, y_{1}, \ldots, y_{n}\right)
$$

é um isomorfismo.

Além disso, esta função induz um isomorfismo isométrico entre $\mathscr{L}\left({ }^{m+n} E, F\right)$ e $\mathscr{L}\left({ }^{m} E, \mathscr{L}\left({ }^{n} E, F\right)\right)$.

Demonstração. É fácil verificar que $\Psi$ está bem definida e que é linear. Para ver que $\Psi$ é injetora, note que se $\Psi(A) \equiv 0$, então para cada $\left(x_{1}, \ldots, x_{m}\right) \in E^{m}$ e cada $\left(y_{1}, \ldots, y_{n}\right) \in E^{n}$ temos:

$$
A\left(x_{1}, \ldots, x_{m}, y_{1}, \ldots, y_{n}\right)=\Psi(A)\left(x_{1}, \ldots, x_{m}\right)\left(y_{1}, \ldots, y_{n}\right)=0\left(y_{1}, \ldots, y_{n}\right)=0
$$

Vejamos agora que $\Psi$ é sobrejetora. Dado $B \in \mathscr{L}_{a}\left(E^{m}, \mathscr{L}_{a}\left({ }^{n} E, F\right)\right.$, defina $A: E^{m+n} \longrightarrow F$ por:

$$
A\left(x_{1}, \ldots, x_{m}, y_{1}, \ldots, y_{n}\right) \doteq B\left(x_{1}, \ldots, x_{m}\right)\left(y_{1}, \ldots, y_{n}\right)
$$

É imediato que $A \in \mathscr{L}_{a}\left({ }^{m+n} E, F\right)$ e, além disso, $\Psi(A)=B$ pois:

$$
\Psi(A)\left(x_{1}, \ldots, x_{m}\right)\left(y_{1}, \ldots, y_{n}\right)=A\left(x_{1}, \ldots, x_{m}, y_{1}, \ldots, y_{n}\right)=B\left(x_{1}, \ldots, x_{m}\right)\left(y_{1}, \ldots, y_{n}\right)
$$

Agora vejamos que $\Psi$ induz uma isometria entre $\mathscr{L}\left({ }^{m+n} E, F\right)$ e $\mathscr{L}\left({ }^{m} E, \mathscr{L}\left({ }^{n} E, F\right)\right)$.

Se $A \in \mathscr{L}\left({ }^{m+n} E, F\right)$, temos:

$$
\begin{aligned}
\|\Psi(A)\| & \doteq \sup _{B_{E^{m}}}\left\|\Psi(A)\left(x_{1}, \ldots, x_{m}\right)\right\| \\
& =\sup _{B_{E^{m}}}\left(\sup _{B_{E^{n}}}\left\|\Psi(A)\left(x_{1}, \ldots, x_{m}\right)\left(y_{1}, \ldots, y_{n}\right)\right\|\right)
\end{aligned}
$$




$$
\begin{aligned}
& =\sup _{B_{E^{m}}}\left(\sup _{B_{E^{n}}}\left\|A\left(x_{1}, \ldots, x_{m}, y_{1}, \ldots, y_{n}\right)\right\|\right) \\
& =\sup _{B_{E^{m+n}}}\left\|A\left(x_{1}, \ldots, x_{m}, y_{1}, \ldots, y_{n}\right)\right\| \\
& =\|A\|
\end{aligned}
$$

Observação 3.1.9. Se considerarmos $E_{1}, \ldots, E_{m}$ espaços de Banach sobre um mesmo corpo $\mathbb{K}$, o espaço $\left(E_{1} \times \ldots \times E_{m},\|\cdot\|_{\infty}\right)$ é um espaço de Banach e os resultados apresentados nesta seção também são válidos para aplicações multilineares definidas no produto cartesiano $E_{1} \times \ldots \times E_{m}$.

Denotaremos por $\mathscr{L}_{a}\left(E_{1}, \ldots, E_{m} ; F\right)$ o espaço de todas as aplicaçôes multilineares da forma $A: E_{1} \times \ldots \times E_{m} \longrightarrow F$. Analogamente à notação adotada, denotaremos por $\mathscr{L}\left(E_{1}, \ldots, E_{m} ; F\right) o$ subespaço das aplicações multilineares de $\mathscr{L}_{a}\left(E_{1}, \ldots, E_{m} ; F\right)$ que são contínuas, tal espaço é munido da norma $\|\cdot\|: \mathscr{L}\left(E_{1}, \ldots, E_{m} ; F\right) \longrightarrow[0,+\infty[$, dada por:

$$
\|A\| \doteq \sup \left\{\left\|A\left(x_{1}, \ldots, x_{m}\right)\right\|: x_{j} \in B_{E_{j}}, \quad 1 \leq j \leq m\right\}=\sup _{x \in B_{E_{1} \times \ldots \times E_{m}}}\|A(x)\| .
$$

\subsection{Multilineares Simétricas e Multilineares Alternadas}

Nesta seção serão introduzidas duas importantes classes de aplicações multilineares para a área de análise. A classe das aplicações multilineares alternadas, que é importante para o estudo de formas alternadas em espaços de Banach, e a classe das aplicações multilineares simétricas, que é importante para definirmos polinômios nestes espaços e para o estudo de holomorfia.

Para cada $m \in \mathbb{N}$, denotaremos por $S_{m}$ o grupo de todas as permutações de $m$ elementos, isto é, $S_{m}=\{\sigma:\{1, \ldots, m\} \longrightarrow\{1, \ldots, m\}: \sigma$ é bijetora $\}$. A seguir recordaremos como é definido o sinal de uma permutação. Para uma leitura mais detalhada sobre grupos de permutações, veja [Her06].

Fixado $m \in \mathbb{N}$, seja $\mathbb{Q}\left[X_{1}, \ldots, X_{m}\right]$ o anel dos polinômios com coeficientes racionais e indeterminadas $X_{1}, \ldots, X_{m}$. Seja $P\left(X_{1}, \ldots, X_{m}\right) \in \mathbb{Q}\left[X_{1}, \ldots, X_{m}\right]$ dado por:

$$
P\left(X_{1}, \ldots, X_{m}\right) \doteq \prod_{1 \leq i<j \leq m}\left(X_{i}-X_{j}\right)
$$

Dada $\sigma \in S_{m}$, considere a ação de $\sigma$ em $P\left(X_{1}, \ldots, X_{m}\right)$ dada por:

$$
(\sigma P)\left(X_{1}, \ldots, X_{m}\right) \doteq \prod_{1 \leq i<j \leq m}\left(X_{\sigma(i)}-X_{\sigma(j)}\right) \in \mathbb{Q}\left[X_{1}, \ldots, X_{m}\right]
$$

Observe que $(\sigma P)\left(X_{1}, \ldots, X_{m}\right)$ tem os mesmos fatores de $P\left(X_{1}, \ldots, X_{m}\right)$, a menos dos sinais: 
Sejam $i_{0}, j_{0} \in\{1, \ldots, m\}$ com $i_{0}<j_{0}$. Como $\sigma$ é permutação, segue que $\sigma\left(i_{0}\right) \neq \sigma\left(j_{0}\right)$. Caso $\sigma\left(i_{0}\right)<\sigma\left(j_{0}\right)$, então o termo $\left(x_{\sigma\left(i_{0}\right)}-x_{\sigma\left(j_{0}\right)}\right)$ é um dos fatores de $P\left(X_{1}, \ldots, X_{m}\right)$. Caso contrário, temos que $-\left(x_{\sigma\left(i_{0}\right)}-x_{\sigma\left(j_{0}\right)}\right)$ é um dos fatores de $P\left(X_{1}, \ldots, X_{m}\right)$. Portanto, concluímos que $(\sigma P)\left(X_{1}, \ldots, X_{m}\right)= \pm P\left(X_{1}, \ldots, X_{m}\right)$.

A partir da ação definida acima, definimos o sinal de $\sigma \in S_{m}$ como $(-1)^{\sigma} \in\{1,-1\}$ que satisfaz a seguinte igualdade:

$$
(\sigma P)\left(X_{1}, \ldots, X_{m}\right)=(-1)^{\sigma} P\left(X_{1}, \ldots, P_{m}\right)
$$

Dizemos que $\sigma$ é uma permutação par se $(-1)^{\sigma}=1$ e dizemos que $\sigma$ é uma permutação ímpar se $(-1)^{\sigma}=-1$.

Além disso, se $\sigma, \tau \in S_{m}$, então $\sigma \tau=\sigma \circ \tau \in S_{m}$ e $(-1)^{\sigma \tau}=(-1)^{\sigma}(-1)^{\tau}$. De fato:

$$
\begin{aligned}
(-1)^{\sigma \tau} P\left(X_{1}, \ldots, X_{m}\right) & =((\sigma \tau) P)\left(X_{1}, \ldots, X_{m}\right) \\
& =\prod_{1 \leq i<j \leq m}\left(X_{\sigma(\tau(i))}-X_{\sigma(\tau(j))}\right) \\
& =(-1)^{\sigma} \prod_{1 \leq i<j \leq m}\left(X_{\tau(i)}-X_{\tau(j)}\right) \\
& =(-1)^{\sigma}(-1)^{\tau} \prod_{1 \leq i<j \leq m}\left(X_{i}-X_{j}\right) \\
& =(-1)^{\sigma}(-1)^{\tau} P\left(X_{1}, \ldots, X_{m}\right) .
\end{aligned}
$$

Logo, $(-1)^{\sigma \tau}=(-1)^{\sigma}(-1)^{\tau}$.

Definição 3.2.1. Sejam $m \in \mathbb{N}$ fixado e $A \in \mathscr{L}_{a}\left({ }^{m} E, F\right)$.

(a) Dizemos que $A \in \mathscr{L}_{a}\left({ }^{m} E, F\right)$ é simétrica se, para cada $\sigma \in S_{m}$ e cada $\left(x_{1}, \ldots, x_{m}\right) \in E^{m}$ :

$$
A\left(x_{\sigma(1)}, \ldots, x_{\sigma(m)}\right)=A\left(x_{1}, \ldots, x_{m}\right) .
$$

(b) Dizemos que $A \in \mathscr{L}_{a}\left({ }^{m} E, F\right)$ é alternada se, para cada $\sigma \in S_{m}$ e cada $\left(x_{1}, \ldots, x_{m}\right) \in E^{m}$ :

$$
A\left(x_{\sigma(1)}, \ldots, x_{\sigma(m)}\right)=(-1)^{\sigma} A\left(x_{1}, \ldots, x_{m}\right) .
$$

Denotaremos por $\mathscr{L}_{a}^{s}\left({ }^{m} E, F\right)$ o subespaço vetorial de $\mathscr{L}_{a}\left({ }^{m} E, F\right)$ formado por todas as aplicaçôes m-lineares simétricas e, analogamente, denotaremos por $\mathscr{L}_{a}^{a}\left({ }^{m} E, F\right)$ o subespaço vetorial de $\mathscr{L}_{a}\left({ }^{m} E, F\right)$ formado por todas as aplicações m-lineares alternadas.

Definimos também os espaços $\mathscr{L}^{s}\left({ }^{m} E, F\right)$ e $\mathscr{L}^{a}\left({ }^{m} E, F\right)$ de maneira natural, isto é:

$$
\mathscr{L}^{s}\left({ }^{m} E, F\right) \doteq \mathscr{L}_{a}^{s}\left({ }^{m} E, F\right) \bigcap \mathscr{L}\left({ }^{m} E, F\right) \quad e \quad \mathscr{L}^{a}\left({ }^{m} E, F\right) \doteq \mathscr{L}_{a}^{a}\left({ }^{m} E, F\right) \bigcap \mathscr{L}\left({ }^{m} E, F\right) .
$$

$E$, no caso em que $F=\mathbb{K}$, escreveremos $\mathscr{L}_{a}^{s}\left({ }^{m} E, \mathbb{K}\right)=\mathscr{L}_{a}^{s}\left({ }^{m} E\right)$ e $\mathscr{L}_{a}^{a}\left({ }^{m} E, \mathbb{K}\right)=\mathscr{L}_{a}^{a}\left({ }^{m} E\right)$. 
Proposição 3.2.2. Dada $A \in \mathscr{L}_{a}\left({ }^{m} E, F\right)$, considere $A^{s} \in \mathscr{L}_{a}^{s}\left(^{m} E, F\right)$ e $A^{a} \in \mathscr{L}_{a}^{a}\left({ }^{m} E, F\right)$ definidas da seguinte maneira:

$$
\begin{gathered}
A^{s}\left(x_{1}, \ldots, x_{m}\right) \doteq \frac{1}{m !} \sum_{\sigma \in S_{m}} A\left(x_{\sigma(1)}, \ldots, x_{\sigma(m)}\right) \\
A^{a}\left(x_{1}, \ldots, x_{m}\right) \doteq \frac{1}{m !} \sum_{\sigma \in S_{m}}(-1)^{\sigma} A\left(x_{\sigma(1)}, \ldots, x_{\sigma(m)}\right)
\end{gathered}
$$

para cada $\left(x_{1}, \ldots, x_{m}\right) \in E^{m}$.

(a) A aplicação $A \stackrel{\Phi}{\longmapsto} A^{s}$ é uma projeção de $\mathscr{L}_{a}\left({ }^{m} E, F\right)$ sobre $\mathscr{L}_{a}^{s}\left({ }^{m} E, F\right)$. Além disso, esta projeção induz uma projeção continua entre $\mathscr{L}\left({ }^{m} E, F\right)$ e $\mathscr{L}^{s}\left({ }^{m} E, F\right)$ tal que $\left\|A^{s}\right\| \leq\|A\|$.

(b) A aplicação $A \stackrel{\Psi}{\longmapsto} A^{a}$ é uma projeção de $\mathscr{L}_{a}\left({ }^{m} E, F\right)$ sobre $\mathscr{L}_{a}^{a}\left({ }^{m} E, F\right)$. Além disso, esta projeção induz uma projeção continua entre $\mathscr{L}\left({ }^{m} E, F\right)$ e $\mathscr{L}^{a}\left({ }^{m} E, F\right)$ tal que $\left\|A^{a}\right\| \leq\|A\|$.

Demonstração. Como as demonstrações dos dois itens são inteiramente análogas, apresentaremos somente a demonstração do item (a).

Vejamos que a aplicação $\Phi$ está bem definida, isto é, que a aplicação $A^{s}$ é simétrica. Para isso, observe que dados $\gamma, \tau \in S_{m}$, existe um $\sigma \in S_{m}$ tal que $\gamma=\sigma \circ \tau$ :

$$
\gamma=\gamma \circ i d=\left(\gamma \circ \tau^{-1}\right) \circ \tau=\sigma \circ \tau
$$

Assim, para cada permutação $\tau \in S_{m}$, temos:

$$
\begin{aligned}
A^{s}\left(x_{\tau(1)}, \ldots, x_{\tau(m)}\right) & \doteq \frac{1}{m !} \sum_{\sigma \in S_{m}} A\left(x_{\sigma \circ \tau(1)}, \ldots, x_{\sigma \circ \tau(m)}\right) \\
& =\frac{1}{m !} \sum_{\gamma \in S_{m}} A\left(x_{\gamma(1)}, \ldots, x_{\gamma(m)}\right) \\
& =A^{s}\left(x_{1}, \ldots, x_{m}\right) .
\end{aligned}
$$

Logo a aplicação $A^{s}$ é, de fato, simétrica e $\Phi$ está bem definida.

Para ver que $\Phi$ é linear, note que, dados $A, B \in \mathscr{L}_{a}\left({ }^{m} E, F\right)$ e $\lambda \in \mathbb{K}$, para cada $\left(x_{1}, \ldots, x_{m}\right)$, temos:

$$
\begin{aligned}
(\lambda A+B)^{s}\left(x_{1}, \ldots, x_{m}\right) & \doteq \frac{1}{m !} \sum_{\sigma \in S_{m}}(\lambda A+B)\left(x_{\sigma(1)}, \ldots, x_{\sigma(m)}\right) \\
& =\frac{1}{m !} \sum_{\sigma \in S_{m}} \lambda A\left(x_{\sigma(1)}, \ldots, x_{\sigma(m)}\right)+B\left(x_{\sigma(1)}, \ldots, x_{\sigma(m)}\right) \\
& =\lambda \frac{1}{m !} \sum_{\sigma \in S_{m}} A\left(x_{\sigma(1)}, \ldots, x_{\sigma(m)}\right)+\frac{1}{m !} \sum_{\sigma \in S_{m}} B\left(x_{\sigma(1)}, \ldots, x_{\sigma(m)}\right)
\end{aligned}
$$




$$
\begin{aligned}
& =\lambda A^{s}\left(x_{1}, \ldots, x_{m}\right)+B^{s}\left(x_{1}, \ldots, x_{m}\right) \\
& =\left(\lambda A^{s}+B^{s}\right)\left(x_{1}, \ldots, x_{m}\right) .
\end{aligned}
$$

Para verificar que $\Phi$ é uma projeção, vejamos que se $A \in \mathscr{L}_{a}^{s}\left({ }^{m} E, F\right)$, então $A^{s}=A$ :

$$
\left(A^{s}\right)^{s}\left(x_{1}, \ldots, x_{m}\right) \doteq \frac{1}{m !} \sum_{\sigma \in S_{m}} A^{s}\left(x_{\sigma(1)}, \ldots, x_{\sigma(m)}\right)=\frac{m !}{m !} A\left(x_{1}, \ldots, x_{m}\right)=A\left(x_{1}, \ldots, x_{m}\right) .
$$

Suponha que $A \in \mathscr{L}\left({ }^{m} E, F\right)$ e seja $\left(x_{1}, \ldots, x_{m}\right) \in B_{E^{m}}$. Então:

$$
\begin{aligned}
\left\|A^{s}\left(x_{1}, \ldots, x_{m}\right)\right\| & =\frac{1}{m !}\left\|\sum_{\sigma \in S_{m}} A\left(x_{\sigma(1)}, \ldots, x_{\sigma(m)}\right)\right\| \\
& \leq \frac{1}{m !} \sum_{\sigma \in S_{m}}\|A\|\left\|x_{\sigma(1)}\right\| \ldots\left\|x_{\sigma(m)}\right\| \\
& =\frac{1}{m !} \sum_{\sigma \in S_{m}}\|A\|\left\|x_{1}\right\| \ldots\left\|x_{m}\right\| \\
& =\frac{m !}{m !}\|A\|\left\|x_{1}\right\| \ldots\left\|x_{m}\right\| \\
& =\|A\|\left\|x_{1}\right\| \ldots\left\|x_{m}\right\| .
\end{aligned}
$$

Logo, tomando o supremo sobre $B_{E^{m}}$, segue que $\left\|A^{s}\right\| \leq\|A\|$ para toda $\left.A \in \mathscr{L}{ }^{m} E, F\right)$. Isso prova que a aplicação $\left.\Phi\right|_{\mathscr{L}\left({ }^{m} E, F\right)}: \mathscr{L}\left(^{m} E, F\right) \longrightarrow \mathscr{L}^{s}\left({ }^{m} E, F\right)$ é contínua e $\|\Phi\| \leq 1$.

Por conveniência, vamos definir os espaços de aplicações multilineares para $m=0$ como o espaço das funções constantes:

$$
\mathscr{L}_{a}\left({ }^{0} E, F\right)=\mathscr{L}_{a}^{a}\left({ }^{0} E, F\right)=\mathscr{L}_{a}^{s}\left({ }^{0} E, F\right)=\mathscr{L}\left({ }^{0} E, F\right)=\mathscr{L}^{a}\left({ }^{0} E, F\right)=\mathscr{L}^{s}\left({ }^{0} E, F\right)=F
$$

Agora nos concentraremos no estudo de aplicações multilineares simétricas e, para isso, apresentaremos algumas definições para simplificar a notação dos próximos resultados.

Definição 3.2.3. Para cada $n \in \mathbb{N}$ e cada multi-índice $\alpha=\left(\alpha_{1}, \ldots, \alpha_{n}\right) \in \mathbb{N}_{0}^{n}$, definimos:

$$
|\alpha| \doteq \alpha_{1}+\ldots+\alpha_{n} \quad \text { e } \quad \alpha ! \doteq \alpha_{1} ! \ldots \alpha_{n} !
$$

Definição 3.2.4. Sejam $A \in \mathscr{L}_{a}\left({ }^{m} E, F\right)$ e $n \in \mathbb{N}$. Para cada $\left(x_{1}, \ldots, x_{n}\right) \in E^{n}$ e cada multi-índice $\alpha=\left(\alpha_{1}, \ldots, \alpha_{n}\right) \in \mathbb{N}_{0}^{n}$ com $|\alpha|=m$, definimos: 


$$
A x_{1}^{\alpha_{1}} \ldots x_{n}^{\alpha_{n}}= \begin{cases}A & \text { se } m=0, \\ A(\underbrace{x_{1}, \ldots, x_{1}}_{\alpha_{1} \text { vezes }}, \ldots, \underbrace{x_{n}, \ldots, x_{n}}_{\alpha_{n} \text { vezes }}) & \text { se } m \geq 1 .\end{cases}
$$

Teorema 3.2.5 (Fórmula de Leibniz). Seja $A \in \mathscr{L}_{a}^{s}\left({ }^{m} E, F\right)$. Para todos $x_{1}, \ldots, x_{n} \in E$, vale:

$$
A\left(x_{1}+\ldots+x_{n}\right)^{m}=\sum_{\substack{\alpha \in \mathbb{N}_{0}^{n} \\|\alpha|=m}} \frac{m !}{\alpha !} A x_{1}^{\alpha_{1}} \ldots x_{n}^{\alpha_{n}} .
$$

Demonstração. Faremos indução em $m$. O resultado é imediato para o caso $m=0$ e, para o caso $m=1$, basta observar que:

$$
\sum_{|\alpha|=1} \frac{1 !}{\alpha !} A x_{1}^{\alpha_{1}} \ldots x_{n}^{\alpha_{n}}=A x_{1}+\ldots+A x_{n}=A\left(x_{1}+\ldots+x_{n}\right)^{1} .
$$

Agora suponha que a fórmula seja válida para toda aplicação $m$-linear simétrica. Vejamos que é válida para toda $A \in \mathscr{L}_{a}^{s}\left({ }^{m+1} E, F\right)$. Para isso utilizaremos o isomorfismo que foi apresentado no Teorema 3.1.8:

$$
\begin{aligned}
A\left(x_{1}+\ldots+x_{n}\right)^{m+1} & =\underbrace{A\left(x_{1}+\ldots+x_{n}\right)}_{\in \mathscr{L}_{a}^{s}(m E, F)}\left(x_{1}+\ldots+x_{n}\right)^{m} \\
& =\sum_{\substack{\alpha \in \mathbb{N}_{0}^{n} \\
|\alpha|=m}} \frac{m !}{\alpha !} A\left(x_{1}+\ldots+x_{n}\right) x_{1}^{\alpha_{1}} \ldots x_{n}^{\alpha_{n}} \\
& =\sum_{\substack{\alpha \in \mathbb{N}_{0}^{n} \\
|\alpha|=m}} \frac{m !}{\alpha !} A(x_{1}+\ldots+x_{m}, \underbrace{x_{1}, \ldots, x_{1}}_{\alpha_{1}}, \ldots, \underbrace{x_{n}, \ldots, x_{n}}_{\alpha_{n}}) \\
& =\sum_{\substack{\alpha \in \mathbb{N}_{0}^{n} \\
|\alpha|=m}} \frac{m !}{\alpha !}\left[A x_{1}^{\alpha_{1}+1} x_{2}^{\alpha_{2}} \ldots x_{n}^{\alpha_{n}}+\ldots+A x_{1}^{\alpha_{1}} x_{2}^{\alpha_{2}} \ldots x_{n}^{\alpha_{n}+1}\right] \\
& =\sum_{\substack{\alpha \in \mathbb{N}_{0}^{n} \\
|\alpha|=m}} \frac{m !}{\alpha !} A x_{1}^{\alpha_{1}+1} x_{2}^{\alpha_{2}} \ldots x_{n}^{\alpha_{n}}+\ldots+\sum_{\substack{\alpha \in \mathbb{N}_{0}^{n} \\
|\alpha|=m}} \frac{m !}{\alpha !} A x_{1}^{\alpha_{1}} x_{2}^{\alpha_{2}} \ldots x_{n}^{\alpha_{n}+1} .
\end{aligned}
$$

Para cada $1 \leq i \leq n$, defina $\beta^{(i)} \doteq\left(\beta_{1}^{(i)}, \ldots, \beta_{n}^{(i)}\right) \in \mathbb{N}_{0}^{n}$ onde $\beta_{j}^{(i)} \doteq \alpha_{j}+\delta_{i j}$ para $1 \leq j \leq n$ e $\delta_{i j}$ é dada por:

$$
\delta_{i j}= \begin{cases}1 & \text { se } j=i \\ 0 & \text { se } j \neq i\end{cases}
$$

Com esta definição, para cada um dos fatores em (1), podemos reindexar o somatório da seguinte maneira: 


$$
\begin{aligned}
\sum_{\substack{\alpha \in \mathbb{N}_{0}^{n} \\
|\alpha|=m}} \frac{m !}{\alpha !} A x_{1}^{\alpha_{1}} \ldots x_{i}^{\alpha_{i}+1} \ldots x_{n}^{\alpha_{n}} & =\sum_{\left|\beta^{(i)}\right|=m+1} \frac{m !}{\beta_{1}^{(i)} ! \ldots\left(\beta_{i}^{(i)}-1\right) ! \ldots \beta_{n}^{(i)} !} A x_{1}^{\beta_{1}^{(i)}} \ldots x_{i}^{\beta_{i}^{(i)}} \ldots x_{n}^{\beta_{n}^{(i)}} \\
& =\sum_{\substack{|\beta|=m+1 \\
\beta_{i} \geq 1}} \frac{m ! \beta_{i}}{\beta_{1} ! \ldots \beta_{i} ! \ldots \beta_{n} !} A x_{1}^{\beta_{1}} \ldots x_{n}^{\beta_{n}} .
\end{aligned}
$$

Observe que percorremos todos os multi-índices $\beta \in \mathbb{N}_{0}^{n}$ com $|\beta|=m+1$ quando percorremos $i=1, \ldots, n$. Logo, de (1) e (2), obtemos:

$$
\begin{aligned}
A\left(x_{1}, \ldots, x_{n}\right)^{m+1} & =\sum_{\substack{\beta \in \mathbb{N}_{0}^{n} \\
|\beta|=m+1}} m !\left(\frac{\beta_{1}}{\beta !}+\ldots+\frac{\beta_{n}}{\beta !}\right) A x_{1}^{\beta_{1}} \ldots x_{n}^{\beta_{n}} \\
& =\sum_{\substack{\beta \in \mathbb{N}_{0}^{n} \\
|\beta|=m+1}} \frac{m !}{\beta !}\left(\beta_{1}+\ldots+\beta_{n}\right) A x_{1}^{\beta_{1}} \ldots x_{n}^{\beta_{n}} \\
& =\sum_{\substack{\beta \in \mathbb{N}_{0}^{n} \\
|\beta|=m+1}} \frac{(m+1) !}{\beta !} A x_{1}^{\beta_{1}} \ldots x_{n}^{\beta_{n}} .
\end{aligned}
$$

Corolário 3.2.6 (Fórmula Binomial). Seja $A \in \mathscr{L}_{a}^{s}\left({ }^{m} E, F\right)$. Então, para todos $x, y \in E$, temos:

$$
A(x+y)^{m}=\sum_{k=0}^{m}\left(\begin{array}{c}
m \\
k
\end{array}\right) A x^{m-k} y^{k}
$$

O teorema a seguir é muito importante para o estudo de aplicações multilineares simétricas, polinômios e funções holomorfas pois nos diz que uma aplicação multilinear simétrica depende apenas dos seus valores na diagonal do espaço $E^{m}$.

Teorema 3.2.7 (Fórmula de Polarização). Seja $A \in \mathscr{L}_{a}^{s}\left({ }^{m} E, F\right)$. Então:

$$
A\left(x_{1}, \ldots, x_{m}\right)=\frac{1}{m ! 2^{m}} \sum_{\substack{\varepsilon_{i}= \pm 1 \\ i=1, \ldots, m}} \varepsilon_{1} \ldots \varepsilon_{m} A\left(x_{0}+\varepsilon_{1} x_{1}+\cdots+\varepsilon_{m} x_{m}\right)^{m}
$$

para quaisquer $x_{0}, \ldots, x_{m} \in E$.

Demonstração. Pela Fórmula de Leibniz, temos:

$$
\begin{aligned}
A\left(x_{0}+\varepsilon_{1} x_{1}+\cdots+\varepsilon_{m} x_{m}\right)^{m} & =\sum_{|\alpha|=m} \frac{m !}{\alpha !} A x_{0}^{\alpha_{0}}\left(\varepsilon_{1} x_{1}\right)^{\alpha_{1}} \ldots\left(\varepsilon_{m} x_{m}\right)^{\alpha_{m}} \\
& =\sum_{|\alpha|=m} \frac{m !}{\alpha !} \varepsilon_{1}^{\alpha_{1}} \ldots \varepsilon_{m}^{\alpha_{m}} A x_{0}^{\alpha_{0}} \ldots x_{m}^{\alpha_{m}} .
\end{aligned}
$$

Tomando o somatório em todos os $\varepsilon_{i}= \pm 1$ com $1 \leq i \leq m$, temos: 


$$
\sum_{\substack{\varepsilon_{i}= \pm 1 \\ i=1, \ldots, m}} \varepsilon_{1} \ldots \varepsilon_{m} A\left(x_{0}+\varepsilon_{1} x_{1}+\cdots+\varepsilon_{m} x_{m}\right)^{m}=\sum_{|\alpha|=m} \frac{m !}{\alpha !} A x_{0}^{\alpha_{0}} \ldots x_{m}^{\alpha_{m}} \sum_{\substack{\varepsilon_{i}= \pm 1 \\ i=1, \ldots, m}} \varepsilon_{1}^{\alpha_{1}+1} \ldots \varepsilon_{m}^{\alpha_{m}+1}
$$

Observe que se $\alpha_{j}=0$ para algum $1 \leq j \leq m$, como $\varepsilon_{j}= \pm 1$, temos:

$$
\sum_{\substack{\varepsilon_{i}= \pm 1 \\ i=1, \ldots, m}} \varepsilon_{1}^{\alpha_{1}+1} \ldots \varepsilon_{j}^{1} \ldots \varepsilon_{m}^{\alpha_{m}+1}=\sum_{\substack{\varepsilon_{i}= \pm 1 \\ i \neq j}} \varepsilon_{1}^{\alpha_{1}+1} \ldots \varepsilon_{m}^{\alpha_{m}+1}-\sum_{\substack{\varepsilon_{i}= \pm 1 \\ i \neq j}} \varepsilon_{1}^{\alpha_{1}+1} \ldots \varepsilon_{m}^{\alpha_{m}+1}=0
$$

Logo, as parcelas que contribuem para o somatório devem ter $\alpha_{j} \neq 0$ para $j=1 \ldots m$. Mas como $|\alpha|=m$, só há uma parcela na somatória, formada pelo multi-índice dado por $\alpha_{0}=0$ e $\alpha_{j}=1$ para $j=1 \ldots m$. Concluímos que:

$$
\sum_{\substack{\varepsilon_{i}= \pm 1 \\ i=1, \ldots, m}} \varepsilon_{1} \ldots \varepsilon_{m} A\left(x_{0}+\varepsilon_{1} x_{1}+\cdots+\varepsilon_{m} x_{m}\right)^{m}=m ! 2^{m} A\left(x_{1}, \ldots, x_{m}\right) .
$$

Proposição 3.2.8. Seja $\left(A_{j}\right) \subset \mathscr{L}_{a}\left({ }^{m} E, F\right)$ uma sequência pontualmente convergente, isto é, $\lim _{j} A_{j}(x)=A(x)$ existe para cada $x \in E^{m}$. Então:

(a) $A \in \mathscr{L}_{a}\left({ }^{m} E, F\right)$.

(b) Se todo $A_{j}$ é simétrico, então A é simétrico.

(c) Se todo $A_{j}$ é alternado, então A é alternado.

(d) Se todo $A_{j}$ é contínuo, então A é contínuo.

Demonstração. (a): Para cada $1 \leq i \leq m$ fixado, dados $x_{1}, \ldots, x_{m}, y_{i} \in E$ e $\lambda \in \mathbb{K}$, temos:

$$
\begin{aligned}
A\left(x_{1}, \ldots, \lambda x_{i}+y_{i}, \ldots, x_{m}\right) & =\lim _{j} A_{j}\left(x_{1}, \ldots, \lambda x_{i}+y_{i}, \ldots, x_{m}\right) \\
& =\lim _{j} \lambda A_{j}\left(x_{1}, \ldots, x_{i}, \ldots, x_{m}\right)+A_{j}\left(x_{1}, \ldots, y_{i}, \ldots, x_{m}\right) \\
& =\lambda \lim _{j} A_{j}\left(x_{1}, \ldots, x_{i}, \ldots, x_{m}\right)+\lim _{j} A_{j}\left(x_{1}, \ldots, y_{i}, \ldots, x_{m}\right) \\
& =\lambda A\left(x_{1}, \ldots, x_{i}, \ldots, x_{m}\right)+A\left(x_{1}, \ldots, y_{i}, \ldots, x_{m}\right) .
\end{aligned}
$$

(b): Dados $x_{1}, \ldots, x_{m} \in E$ e $\sigma \in S_{m}$, temos:

$$
\begin{aligned}
A\left(x_{\sigma(1)}, \ldots, x_{\sigma(m)}\right) & =\lim _{j} A_{j}\left(x_{\sigma(1)}, \ldots, x_{\sigma(m)}\right) \\
& =\lim _{j} A_{j}\left(x_{1}, \ldots, x_{m}\right) \\
& =A\left(x_{1}, \ldots, x_{m}\right) .
\end{aligned}
$$


(c): Dados $x_{1}, \ldots, x_{m} \in E$ e $\sigma \in S_{m}$, temos:

$$
\begin{aligned}
A\left(x_{\sigma(1)}, \ldots, x_{\sigma(m)}\right) & =\lim _{j} A_{j}\left(x_{\sigma(1)}, \ldots, x_{\sigma(m)}\right) \\
& =\lim _{j}(-1)^{\sigma} A_{j}\left(x_{1}, \ldots, x_{m}\right) \\
& =(-1)^{\sigma} A\left(x_{1}, \ldots, x_{m}\right) .
\end{aligned}
$$

(d): Pelo Teorema 3.1.4, basta provar que $A$ é contínua em cada variável. Mostraremos, sem perda de generalidade, que $A$ é contínua na última variável. Fixados $x_{1}, \ldots, x_{m-1} \in E$, considere a aplicação $A^{x_{1}, \ldots, x_{m-1}}\left(x_{m}\right) \doteq A\left(x_{1}, \ldots, x_{m}\right)$. Note que:

$$
A^{x_{1}, \ldots, x_{m-1}}\left(x_{m}\right)=A\left(x_{1}, \ldots, x_{m}\right)=\lim _{j} A_{j}\left(x_{1}, \ldots, x_{m}\right)=\lim _{j} A_{j}^{x_{1}, \ldots, x_{m-1}}\left(x_{m}\right) .
$$

onde, pelo Teorema 3.1.4, cada $A_{j}^{x_{1}, \ldots, x_{m-1}}$ é linear e contínuo.

Para cada $x_{m} \in E$, a sequência $\left(A_{j}^{x_{1}, \ldots, x_{m-1}}\left(x_{m}\right)\right)_{j \in \mathbb{N}}$ é convergente, logo, limitada. Pelo Teorema de Banach-Steinhaus, $\sup _{j \in \mathbb{N}}\left\|A_{j}^{x_{1}, \ldots, x_{m-1}}\right\|<\infty$, assim:

$$
\left\|A^{x_{1}, \ldots, x_{m-1}}\left(x_{m}\right)\right\|=\left\|\lim _{j} A_{j}^{x_{1}, \ldots, x_{m-1}}\left(x_{m}\right)\right\| \leq \sup _{j \in \mathbb{N}}\left\|A_{j}^{x_{1}, \ldots, x_{m-1}}\right\|\left\|x_{m}\right\| .
$$

Logo, $A$ é contínua na última variável e segue que $A$ é contínua.

Corolário 3.2.9. $\mathscr{L}^{s}\left({ }^{m} E, F\right)$ e $\mathscr{L}^{a}\left({ }^{m} E, F\right)$ são espaços de Banach.

Podemos gerar aplicações multilineares a partir de formas lineares da seguinte maneira. Dados $\varphi_{1}, \ldots, \varphi_{m} \in E^{*}$, definimos $A \in \mathscr{L}_{a}\left({ }^{m} E\right)$ por

$$
A\left(x_{1}, \ldots, x_{m}\right) \doteq \varphi_{1}\left(x_{1}\right) \ldots \varphi_{m}\left(x_{m}\right),
$$

para cada $x_{1}, \ldots, x_{m} \in E$. Esta idéia é generalizada a seguir.

Definição 3.2.10. Dados $A \in \mathscr{L}_{a}\left({ }^{m} E\right)$ e $B \in \mathscr{L}_{a}\left({ }^{n} E\right)$ o produto tensorial $A \otimes B \in \mathscr{L}_{a}\left({ }^{m+n} E\right)$ é definido por:

$$
(A \otimes B)\left(x_{1}, \ldots, x_{m+n}\right) \doteq A\left(x_{1}, \ldots, x_{m}\right) B\left(x_{m+1}, \ldots, x_{m+n}\right)
$$

para todos $x_{1}, \ldots, x_{m+n} \in E$.

O próximo resultado lista algumas propriedades básicas do produto tensorial e sua demonstração é imediata.

Proposição 3.2.11. Sejam $A_{1} \in \mathscr{L}_{a}\left({ }^{m} E\right), A_{2} \in \mathscr{L}_{a}\left({ }^{n} E\right)$ e $A_{3} \in \mathscr{L}_{a}\left({ }^{k} E\right)$. Então

(a) Se $A_{1}$ e $A_{2}$ são contínuos, então $A_{1} \otimes A_{2}$ é contínuo. 
(b) A aplicação $\left(A_{1}, A_{2}\right) \longmapsto A_{1} \otimes A_{2}$ é bilinear.

(c) $\left(A_{1} \otimes A_{2}\right) \otimes A_{3}=A_{1} \otimes\left(A_{2} \otimes A_{3}\right)$

Observação 3.2.12. Também é possível definir o produto tensorial $A \otimes B$ para o caso em que uma das aplicações tem imagem em um espaço de Banach qualquer.

\subsection{Polinômios e Polinômios Homogêneos}

Nesta seção trataremos de estudar uma classe importante de funções entre espaços de Banach. Apresentaremos os resultados básicos da teoria de polinômios e polinômios homogêneos para que, posteriormente, possamos estudar funções analíticas e problemas de extensão.

Definição 3.3.1. Seja $m \in \mathbb{N}$. Dizemos que uma função $P: E \longrightarrow F$ é um polinômio $m$-homogêneo se existe $A \in \mathscr{L}_{a}\left({ }^{m} E, F\right)$ tal que $P(x)=A x^{m}$ para cada $x \in E$.

Denotaremos por $\mathscr{P}_{a}\left({ }^{m} E, F\right)$ o espaço vetorial de todos os polinômios m-homogêneos de $E$ em F. E, conforme a notação definida para aplicaçôes multilineares, denotaremos por $\mathscr{P}\left({ }^{m} E, F\right)$ o subespaço de $\mathscr{P}_{a}\left({ }^{m} E, F\right)$ formado pelos polinômios m-homogêneos que são contínuos em relação às normas de $E$ e de $F$.

Quando $F=\mathbb{K}$, escreveremos $\mathscr{P}_{a}\left({ }^{m} E, F\right)=\mathscr{P}_{a}\left({ }^{m} E\right)$ e $\mathscr{P}\left({ }^{m} E, F\right)=\mathscr{P}\left({ }^{m} E\right)$.

Exemplo 3.3.2. Se $E=\mathbb{K}$, dada $A \in \mathscr{L}_{a}\left({ }^{m} E, F\right)$, seja $a=A(1, \ldots, 1)$. Então, para cada $\left(x_{1}, \ldots, x_{m}\right) \in E^{m}$ :

$$
A\left(x_{1}, \ldots, x_{m}\right)=x_{1} \ldots x_{m} A(1, \ldots, 1) \doteq a x_{1} \ldots x_{m}
$$

Assim, todo polinômio $m$-homogêneo $P: \mathbb{K} \longrightarrow F$ é da forma $P(x)=a x^{m}$ com a $\in F$. Além disso, se tomarmos $E=F=\mathbb{K}$, obtemos a definição clássica de polinômios m-homogêneos.

Pretendemos relacionar o espaço dos polinômios $m$-homogêneos com o espaço das aplicações $m$-lineares simétricas. Para isso utilizaremos a seguinte notação:

Definição 3.3.3. Dada $A \in \mathscr{L}_{a}\left({ }^{m} E, F\right)$, definimos $\widehat{A} \in \mathscr{P}_{a}\left({ }^{m} E, F\right)$ por:

$$
\widehat{A}(x) \doteq A(x, \ldots, x) \text {, para cada } x \in E \text {. }
$$

Teorema 3.3.4. A aplicação $\Phi: \mathscr{L}_{a}^{s}\left({ }^{m} E, F\right) \longrightarrow \mathscr{P}_{a}\left({ }^{m} E, F\right)$ dada por $\Phi(A) \doteq \widehat{A}$ é um isomorfismo de espaços vetorias.

Demonstração. Vejamos que $\Phi$ é linear. Dados $A_{1}, A_{2} \in \mathscr{L}_{a}^{s}\left({ }^{m} E, F\right)$ e $\lambda \in \mathbb{K}$, para cada $x \in E$, temos:

$$
\Phi\left(\lambda A_{1}+A_{2}\right)(x)=\left(\lambda \widehat{A_{1}+A_{2}}\right)(x) \doteq\left(\lambda A_{1}+A_{2}\right) x^{m}=\lambda A_{1} x^{m}+A_{2} x^{m}=\left(\lambda \widehat{A_{1}}+\widehat{A}_{2}\right)(x) .
$$


Dado $P \in \mathscr{P}_{a}\left({ }^{m} E, F\right)$, existe $A \in \mathscr{L}_{a}\left({ }^{m} E, F\right)$ tal que $P=\widehat{A}$. Considere a aplicação $A^{s}$, como na Proposição 3.2.2 . Então, para cada $x \in E$ :

$$
\widehat{A^{s}}(x) \doteq A^{s}(x, \ldots, x) \doteq \frac{1}{m !} \sum_{\sigma \in S_{m}} A x^{m}=A x^{m}=P(x)
$$

Logo, a aplicação $\Phi$ é sobrejetora. Agora vejamos que $\Phi$ é injetora. Para isso, suponha que $A, B \in \mathscr{L}_{a}^{s}\left({ }^{m} E, F\right)$ são tais que $\widehat{A}=P=\widehat{B}$. Então, dados $x_{1}, \ldots, x_{m} \in E$, segue da Fórmula de Polarização com $x_{0}=0$, que:

$$
\begin{aligned}
A\left(x_{1}, \ldots, x_{m}\right) & =\frac{1}{m ! 2^{m}} \sum_{\substack{\varepsilon_{i}= \pm 1 \\
i=1, \ldots, m}} \varepsilon_{1} \ldots \varepsilon_{m} A\left(\varepsilon_{1} x_{1}+\cdots+\varepsilon_{m} x_{m}\right)^{m} \\
& =\frac{1}{m ! 2^{m}} \sum_{\substack{\varepsilon_{i}= \pm 1 \\
i=1, \ldots, m}} \varepsilon_{1} \ldots \varepsilon_{m} P\left(\varepsilon_{1} x_{1}+\cdots+\varepsilon_{m} x_{m}\right) \\
& =\frac{1}{m ! 2^{m}} \sum_{\substack{\varepsilon_{i}= \pm 1 \\
i=1, \ldots, m}} \varepsilon_{1} \ldots \varepsilon_{m} B\left(\varepsilon_{1} x_{1}+\cdots+\varepsilon_{m} x_{m}\right)^{m} \\
& =B\left(x_{1}, \ldots, x_{m}\right) .
\end{aligned}
$$

Isso mostra que $\Phi$ é injetora e segue que é um isomorfismo entre espaços vetorias.

Observação 3.3.5. No caso em que $m=0$, indentificaremos $\mathscr{P}_{a}\left({ }^{0} E, F\right) \cong \mathscr{L}_{a}^{s}\left({ }^{0} E, F\right)=F$ como o espaço das aplicaçôes constantes.

A seguir apresentamos um critério para a continuidade de polinômios $m$-homogêneos que é semelhante ao apresentado no Teorema 3.1.2.

Teorema 3.3.6. Dado $P \in \mathscr{P}_{a}\left({ }^{m} E, F\right)$, seja $A \in \mathscr{L}_{a}^{s}\left({ }^{m} E, F\right)$ tal que $P=\widehat{A}$. São equivalentes:
(a) $A \in \mathscr{L}^{s}\left({ }^{m} E, F\right)$
(b) $P \in \mathscr{P}\left({ }^{m} E, F\right)$
(c) P é contínuo na origem
(d) Existe uma constante $M>0$ tal que, para cada $x \in E,\|P(x)\| \leq M\|x\|^{m}$

Demonstração. As implicações (a) $\Longrightarrow(\mathrm{b}) \Longrightarrow(\mathrm{c})$ são imediatas.

(c) $\Longrightarrow$ (d): Pela continuidade na origem, existe um $\delta>0$ tal que $\|P(y)\| \leq 1$ sempre que $\|y\| \leq \delta$. Dado $x \neq 0$ qualquer, o ponto $z \doteq \frac{\delta}{2\|x\|}$ é tal que:

$$
\left(\frac{\delta}{2\|x\|}\right)^{m}\|P(x)\|=\|P(z)\| \leq 1 .
$$


Portanto, tomando $M \doteq\left(\frac{2}{\delta}\right)^{m}$, segue que $\|P(x)\| \leq M\|x\|^{m}$.

$(\mathrm{d}) \Longrightarrow$ (a): Dado $\left(x_{1}, \ldots, x_{m}\right) \in B_{E^{m}}$, então $\left\|x_{i}\right\| \leq 1$ para todo $1 \leq i \leq m$ e, pela Fórmula de Polarização com $x_{0}=0$, temos:

$$
\begin{aligned}
\left\|A\left(x_{1}, \ldots, x_{m}\right)\right\| & \leq \frac{1}{m ! 2^{m}} \sum_{\substack{\varepsilon_{i}= \pm 1 \\
i=1, \ldots, m}}\left|\varepsilon_{1}\right| \ldots\left|\varepsilon_{m}\right|\left\|A\left(\varepsilon_{1} x_{1}+\cdots+\varepsilon_{m} x_{m}\right)^{m}\right\| \\
& =\frac{1}{m ! 2^{m}} \sum_{\substack{\varepsilon_{i}= \pm 1 \\
i=1, \ldots, m}}\left|\varepsilon_{1}\right| \ldots\left|\varepsilon_{m}\right|\left\|P\left(\varepsilon_{1} x_{1}+\cdots+\varepsilon_{m} x_{m}\right)\right\| \\
& \leq \frac{1}{m ! 2^{m}} \sum_{\substack{\varepsilon_{i}= \pm 1 \\
i=1, \ldots, m}} M\left(\left\|x_{1}\right\|+\cdots+\left\|x_{m}\right\|\right)^{m} \\
& \leq \frac{1}{m ! 2^{m}} \sum_{\substack{\varepsilon_{i}= \pm 1 \\
i=1, \ldots, m}} M m^{m} \\
& =\frac{M m^{m}}{m !} .
\end{aligned}
$$

E segue que $A$ é contínuo.

Assim como no caso das aplicações multilineares, o teorema anterior nos mostra que polinômios $m$-homogêneos contínuos são limitados sobre os subconjuntos limitados de $E$. Isto nos permite definir a seguinte função:

$$
\|\cdot\|: \mathscr{P}\left({ }^{m} E, F\right) \longrightarrow\left[0,+\infty\left[\text { dada por }\|P\| \doteq \sup _{\|x\| \leq 1}\|P(x)\| .\right.\right.
$$

Segue também do Teorema 3.3.6 que um polinômio $P$ é contínuo se, e somente se, $\|P\|<\infty$. Além disso, esta é a menor constante que satisfaz 3.3.6(d):

Dado $x \in E$, temos $\left\|P\left(\frac{x}{\|x\|}\right)\right\| \leq\|P\|$ e obtemos:

$$
\|P(x)\| \leq\|P\|\|x\|^{m}
$$

Isto é, $\|P\|$ satisfaz a condição 3.3.6(d). Por outro lado, se $M>0$ é uma constante que satisfaz 3.3.6(d), dado $x \in B_{E}$, temos $\|P(x)\| \leq M$. Assim segue que $\|P\| \leq M$ e podemos concluir que:

$$
\|P\|=\inf \left\{M>0:\|P(x)\| \leq M\|x\|^{m} \quad \forall x \in E\right\} .
$$

Proposição 3.3.7. A aplicação $\|\cdot\|$ é uma norma em $\mathscr{P}\left({ }^{m} E, F\right)$.

Demonstração. A demonstração é análoga à demonstração da Proposição 3.1.6.

Teorema 3.3.8. A aplicação $\Phi: \mathscr{L}^{s}\left({ }^{m} E, F\right) \longrightarrow \mathscr{P}\left({ }^{m} E, F\right)$ dada por $\Phi(A) \doteq \widehat{A}$ é um isomorfismo 
topológico de espaços normados. Além disso,

$$
\|\widehat{A}\| \leq\|A\| \leq \frac{m^{m}}{m !}\|\widehat{A}\| .
$$

Demonstração. Pelo Teorema 3.3.4, $\Phi$ é linear e injetora. Além disso, pelo Teorema 3.3.6 , $\Phi$ é sobrejetora. Para cada $x \in E$, temos:

$$
\|\widehat{A}(x)\|=\left\|A x^{m}\right\| \leq\|A\|\|x\|^{m} .
$$

Logo, $\|\widehat{A}\| \leq\|A\|$. Dados $x_{1}, \ldots, x_{m} \in B_{E}$ :

$$
\begin{aligned}
\left\|A\left(x_{1}, \ldots, x_{m}\right)\right\| & \leq \frac{1}{m ! 2^{m}} \sum_{\substack{\varepsilon_{i}= \pm 1 \\
i=1, \ldots, m}}\left|\varepsilon_{1}\right| \ldots\left|\varepsilon_{m}\right|\left\|A\left(\varepsilon_{1} x_{1}+\cdots+\varepsilon_{m} x_{m}\right)^{m}\right\| \\
& \leq \frac{1}{m ! 2^{m}} \sum_{\substack{\varepsilon_{i}= \pm 1 \\
i=1, \ldots, m}}\left\|\widehat{A}\left(\varepsilon_{1} x_{1}+\cdots+\varepsilon_{m} x_{m}\right)\right\| \\
& \leq \frac{1}{m ! 2^{m}} \sum_{\substack{\varepsilon_{i}= \pm 1 \\
i=1, \ldots, m}}\|\widehat{A}\|\left\|\varepsilon_{1} x_{1}+\cdots+\varepsilon_{m} x_{m}\right\|^{m} \\
& \leq \frac{m^{m}}{m !}\|\widehat{A}\| .
\end{aligned}
$$

Portanto, $\|A\| \leq \frac{m^{m}}{m !}\|\widehat{A}\|$.

Corolário 3.3.9. $\mathscr{P}\left({ }^{m} E, F\right)$ munido da norma $\|\cdot\|$ é um espaço de Banach.

A seguir apresentaremos uma generalização do Teorema de Banach-Steinhaus para polinômios homogêneos. Para isso precisaremos dos seguintes lemas:

Lema 3.3.10. Seja $P \in \mathscr{P}_{a}\left({ }^{m} E, F\right)$. Se $P$ é limitado por $c>0$ sobre uma bola $B(a, r)$, então $P$ é limitado por $\frac{\mathrm{cm}^{m}}{\mathrm{~m} !}$ sobre $B(0, r)$.

Demonstração. Seja $A \in \mathscr{L}_{a}^{s}\left({ }^{m} E, F\right)$ tal que $P=\widehat{A}$. Pela Fórmula de Polarização, temos:

$$
A\left(x_{1}, \ldots, x_{m}\right)=\frac{1}{m ! 2^{m}} \sum_{\substack{\varepsilon_{i}= \pm 1 \\ i=1, \ldots, m}} \varepsilon_{1} \ldots \varepsilon_{m} A\left(x_{0}+\varepsilon_{1} x_{1}+\cdots+\varepsilon_{m} x_{m}\right)^{m}
$$

Para cada $x \in B(0, r)$, tomando $x_{0}=a$ e $x_{1}=\cdots=x_{m}=\frac{x}{m}$, segue:

$$
\begin{aligned}
P(x) & =A x^{m}=m^{m} A\left(\frac{x}{m}, \ldots, \frac{x}{m}\right) \\
& =m^{m}\left[\frac{1}{m ! 2^{m}} \sum_{\substack{\varepsilon_{i}= \pm 1 \\
i=1, \ldots, m}} \varepsilon_{1} \ldots \varepsilon_{m} A\left(a+\left(\varepsilon_{1}+\cdots+\varepsilon_{m}\right) \frac{x}{m}\right)^{m}\right]
\end{aligned}
$$




$$
=m^{m}\left[\frac{1}{m ! 2^{m}} \sum_{\substack{\varepsilon_{i}= \pm 1 \\ i=1, \ldots, m}} \varepsilon_{1} \ldots \varepsilon_{m} P\left(a+\left(\varepsilon_{1}+\cdots+\varepsilon_{m}\right) \frac{x}{m}\right)\right]
$$

Como, para cada escolha de sinais, $a+\left(\varepsilon_{1}+\cdots+\varepsilon_{m}\right) \frac{x}{m} \in B(a, r)$, pela desigualdade triangular segue que $\|P(x)\| \leq \frac{c m^{m}}{m !}$.

Lema 3.3.11. Sejam $U \subset E$ aberto e $\left(f_{i}\right)$ uma familia de funções contínuas $f_{i}: U \rightarrow F$. Se $\left(f_{i}\right)$ é pontualmente limitada em $U$, então existe um aberto $V \subset U$ tal que $\left(\left.f_{i}\right|_{V}\right)$ é uniformemente limitada.

Demonstração. Para cada $n \in \mathbb{N}$, defina $A_{n} \doteq\left\{x \in U:\left\|f_{i}(x)\right\| \leq n \forall i\right\}$. Como $\left(f_{i}\right)$ é pontualmente limitada, segue que $U=\bigcup_{n \in \mathbb{N}} A_{n}$. Além disso, como $A_{n}=\bigcap_{i}\left(\|\cdot\| \circ f_{i}\right)^{-1}[0, n]$, segue que todo $A_{n}$ é fechado.

Pelo Teorema de Baire, existe $k \in \mathbb{N}$ tal que $\operatorname{int}\left(A_{k}\right) \neq \emptyset$. Defina $V \doteq \operatorname{int}\left(A_{k}\right)$. Deste modo, dado $x \in V$, para cada $i$ :

$$
\left\|f_{i}(x)\right\| \leq \sup _{i}\left\|f_{i}(x)\right\| \leq k .
$$

Teorema 3.3.12. Um subconjunto de $\mathscr{P}\left({ }^{m} E, F\right)$ é limitado se, e somente se, é pontualmente limitado.

Demonstração. Suponha que $\mathscr{A} \subset \mathscr{P}\left({ }^{m} E, F\right)$ seja limitado. Dado $P \in \mathscr{A}$, para cada $x \in E$ :

$$
\|P(x)\| \leq\|P\|\|x\|^{m} \leq \sup _{\mathscr{A}}\|P\|\|x\|^{m}
$$

Logo $\mathscr{A}$ é pontualmente limitado.

Reciprocamente, seja $\left(P_{i}\right) \subset \mathscr{P}\left({ }^{m} E, F\right)$ pontualmente limitada . Pelo Lema 3.3.11 existe uma bola $B(a, r)$ tal que a família $\left(\left.P_{i}\right|_{B(a, r)}\right)$ é uniformemente limitada por uma constante $c>0$. Pelo Lema 3.3.10, $\left(P_{i}\right)$ é uniformemente limitada por $\frac{\mathrm{cm}^{\mathrm{m}}}{\mathrm{m} !}$ em $B(0, r)$.

Dado $x \in B_{E}$, para todo $i$ :

$$
\left\|P_{i}(x)\right\|=\frac{2^{m}}{r^{m}}\left\|P_{i}\left(\frac{r}{2} x\right)\right\| \leq \frac{2^{m} c m^{m}}{r^{m} m !} .
$$

Logo $\sup _{i}\left\|P_{i}\right\| \leq \frac{2^{m} \mathrm{~cm}^{m}}{r^{m} m !}$ e segue que $\left(P_{i}\right)$ é limitada.

Definição 3.3.13. Dizemos que uma função $P: E \longrightarrow F$ é um polinômio de $E$ em $F$ se existe $m \in \mathbb{N}$ tal que a função pode ser representada como $P=P_{0}+P_{1}+\cdots+P_{m} \operatorname{com} P_{j} \in \mathscr{P}_{a}\left({ }^{j} E, F\right)$ para cada $0 \leq j \leq m$.

Se $P \neq 0$ e $m>0$, dizemos que o grau de $P$ é $m$. Denotaremos por $\mathscr{P}_{a}(E, F)$ o espaço vetorial de todos os polinômios de E em F. Analogamente à notação definida para aplicaçôes multilineares, 
denotaremos por $\mathscr{P}(E, F)$ o subespaço de $\mathscr{P}_{a}(E, F)$ formado pelos polinômios que são contínuos em relação às normas de $E$ e de $F$. No caso em que $F=\mathbb{K}$, escreveremos $\mathscr{P}_{a}(E, F)=\mathscr{P}_{a}(E)$ e $\mathscr{P}(E, F)=\mathscr{P}(E)$.

\section{Proposição 3.3.14.}

(a) $\mathscr{P}_{a}(E, F)=\bigoplus_{m \in \mathbb{N}_{0}} \mathscr{P}_{a}\left({ }^{m} E, F\right)$, como soma direta algébrica.

(b) $\mathscr{P}(E, F)=\bigoplus_{m \in \mathbb{N}_{0}} \mathscr{P}\left({ }^{m} E, F\right)$, como soma direta algébrica.

Demonstração. (a): Suponha que $0=P_{0}+\cdots+P_{m} \operatorname{com} P_{j} \in \mathscr{P}_{a}\left({ }^{j} E, F\right)$ para todo $0 \leq j \leq m$. Vamos provar que, para todo $0 \leq j \leq m, P_{j}=0$.

Dados $x \in E$ e $\lambda \in \mathbb{K}, \lambda \neq 0$, temos:

$$
0=\left(\sum_{j=0}^{m} P_{j}\right)(\lambda x)=\sum_{j=0}^{m} P_{j}(\lambda x)=\sum_{j=0}^{m} \lambda^{j} P_{j}(x) .
$$

Dividindo por $\lambda^{m}$, obtemos:

$$
P_{m}(x)+\frac{1}{\lambda} P_{m-1}(x)+\cdots+\frac{1}{\lambda^{m}} P_{0}(x)=0 .
$$

Tomando o limite $|\lambda| \rightarrow \infty$, obtemos $P_{m}=0$. Procedendo indutivamente com o mesmo argumento, obtemos $0=P_{0}=\cdots=P_{m}$.

(b): Pelo que acabamos de provar em (a), basta provar que se $P$ é um polinômio contínuo com representação $P=P_{0}+\cdots+P_{m}$, então $P_{j} \in \mathscr{P}\left({ }^{j} E, F\right)$ para todo $0 \leq j \leq m$. Provaremos isto por indução em $m$.

Se $m=0$, temos que $P=P_{0}$ é contínuo. Suponha que todo polinômio contínuo de grau (no máximo) $m-1$ se escreve como soma de polinômios homogêneos contínuos. Dados $x \in E$ e $\lambda \in \mathbb{K}$, temos:

$$
P(\lambda x)-\lambda^{m} P(x)=\sum_{j=0}^{m} \lambda^{j} P_{j}(x)-\lambda^{m} \sum_{j=0}^{m} P_{j}(x)=\sum_{j=0}^{m-1}\left(\lambda^{j}-\lambda^{m}\right) P_{j}(x) .
$$

Fixe $\lambda \in \mathbb{K}$ tal que $\lambda^{j}-\lambda^{m} \neq 0$ para todo $0 \leq j \leq m-1$. Defina $Q: E \longrightarrow F$ por $Q(x)=P(\lambda x)-\lambda^{m} P(x)$ para cada $x \in E$. Deste modo, $Q$ é um polinômio contínuo de grau $m-1$ e, pela hipótese de indução, temos que $P_{j}$ é contínuo para todo $0 \leq j \leq m-1$. Logo $P_{m}=P-\left(P_{0}+\cdots+P_{m-1}\right)$ é contínuo. 


\section{Capítulo 4}

\section{Extensões de Polinômios em Espaços de}

\section{Banach}

Neste capítulo serão apresentadas extensões de aplicações multilineares e de polinômios entre espaços de Banach para que, posteriormente, possamos estender funções analíticas nestes espaços. Os resultados contidos neste capítulo são baseados em [Dan13].

\subsection{Extensão de Operadores Lineares}

Definição 4.1.1. Sejam $E$ e $F$ espaços de Banach e $T \in \mathscr{L}(E, F)$. O operador $T^{\prime}: F^{\prime} \longrightarrow E^{\prime}$, dado por $T^{\prime}(\varphi) \doteq \varphi \circ T$ é chamado de operador adjunto de $T$.

Proposição 4.1.2. Se $T \in \mathscr{L}(E, F)$, então $T^{\prime} \in \mathscr{L}\left(F^{\prime}, E^{\prime}\right)$ e $\|T\|=\left\|T^{\prime}\right\|$. Além disso, se $T$ é um isomorfismo isométrico, então $T^{\prime}$ também o é.

Demonstração. Dados $\varphi_{1}, \varphi_{2} \in F^{\prime}$ e $\lambda \in \mathbb{K}$, para cada $x \in E$ :

$T^{\prime}\left(\lambda \varphi_{1}+\varphi_{2}\right)(x)=\left(\left(\lambda \varphi_{1}+\varphi_{2}\right) \circ T\right)(x)=\left(\lambda\left(\varphi_{1} \circ T\right)+\varphi_{2} \circ T\right)(x)=\left[\lambda T^{\prime}\left(\varphi_{1}\right)+T^{\prime}\left(\varphi_{2}\right)\right](x)$.

Logo, $T^{\prime}$ é linear. Vejamos que $\|T\|=\left\|T^{\prime}\right\|$. Para cada $\varphi \in F^{\prime}$ :

$$
\|\underbrace{T^{\prime}(\varphi)}_{\in E^{\prime}}\|=\sup _{x \in B_{E}}\left|T^{\prime}(\varphi)(x)\right|=\sup _{x \in B_{E}}|\varphi(T(x))| \leq\|\varphi\| \sup _{x \in B_{E}}\|T(x)\|=\|\varphi\|\|T\| .
$$

Logo, $\left\|T^{\prime}\right\| \leq\|T\|$. Por outro lado, como consequência do Corolário 2.1.3, para cada $x \in E$ :

$$
\|T(x)\|=\sup _{\varphi \in B_{F^{\prime}}}|\varphi(T(x))|=\sup _{\varphi \in B_{F^{\prime}}}\left|T^{\prime}(\varphi)(x)\right| \leq\|x\| \sup _{\varphi \in B_{F^{\prime}}}\left\|T^{\prime}(\varphi)\right\|=\left\|T^{\prime}\right\|\|x\| .
$$

Logo $\|T\| \leq\left\|T^{\prime}\right\|$ e, portanto, $\|T\|=\left\|T^{\prime}\right\|$. 
Agora suponha que $T$ seja um isomorfismo. Dado $\varphi \in \operatorname{ker}\left(T^{\prime}\right)$, para cada $x \in E$, temos:

$$
0=T^{\prime}(\varphi)(x)=\varphi(T(x))
$$

Como $T$ é sobrejetor, segue que $\varphi=0$ e, portanto, $T^{\prime}$ é injetor. Dado $\psi \in E^{\prime}$, observe que $\varphi \doteq \psi \circ T^{-1} \in F^{\prime}$ é tal que $T^{\prime}(\varphi)=\psi$, assim, $T^{\prime}$ é sobrejetor.

Por último, suponha que $T$ seja um isomorfismo isométrico. Para cada $\varphi \in F^{\prime}$, temos:

$$
\left\|T^{\prime}(\varphi)\right\|=\sup _{x \in B_{E}}\left|T^{\prime}(\varphi)(x)\right|=\sup _{x \in B_{E}}|\varphi(T(x))|=\sup _{T(x) \in B_{F}}|\varphi(T(x))|=\|\varphi\| .
$$

O resultado anterior nos permite definir o operador adjunto de $T^{\prime} \in \mathscr{L}\left(F^{\prime}, E^{\prime}\right)$ de maneira natural, isto é, definimos $T^{\prime \prime} \in \mathscr{L}\left(E^{\prime \prime}, F^{\prime \prime}\right)$ por $T^{\prime \prime} \doteq\left(T^{\prime}\right)^{\prime}$.

Lembre que denotamos a inclusão canônica de um espaço de Banach $E$ no bidual $E^{\prime \prime}$ por $J_{E}: E \longrightarrow E^{\prime \prime}$, e esta aplicação é dada por $J_{E}(x)=\widehat{x}$, onde $\widehat{x}\left(x^{\prime}\right) \doteq x^{\prime}(x)$, para cada $x^{\prime} \in E^{\prime}$. Esta inclusão é uma imersão isométrica e, por este motivo, identificamos $E=J_{E}(E) \subset E^{\prime \prime}$.

O próximo resultado nos mostra qual a relação entre $T$ e $T^{\prime \prime}$ quando consideradas as identificações $E \subset E^{\prime \prime}$ e $F \subset F^{\prime \prime}$.

Teorema 4.1.3. Dado $T \in \mathscr{L}(E, F)$, o operador $T^{\prime \prime} \in \mathscr{L}\left(E^{\prime \prime}, F^{\prime \prime}\right)$ é uma extensão de $T$ no sentido de que $T^{\prime \prime} \circ J_{E}=J_{F} \circ T$.

Demonstração. Dados $x \in E$ e $\varphi \in F^{\prime}$, temos:

$\left[\left(T^{\prime \prime} \circ J_{E}\right)(x)\right](\varphi)=T^{\prime \prime}(\widehat{x})(\varphi)=\left(\widehat{x} \circ T^{\prime}\right)(\varphi)=\widehat{x}(\varphi \circ T)=(\varphi \circ T)(x)=J_{F}(T(x))(\varphi)=\left[\left(J_{F} \circ T\right)(x)\right](\varphi)$.

Observação 4.1.4. Segundo o resultado anterior, dizer que o operador $T^{\prime \prime}$ é uma extensão de $T$ ao bidual é o mesmo que dizer que o seguinte diagrama é comutativo:

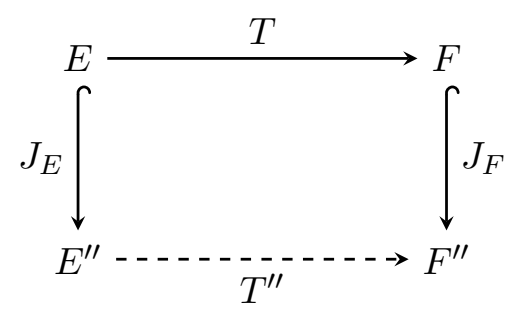

Além disso, segundo o teorema anterior, também vale que:

(a) $T^{\prime \prime}\left(J_{E}(E)\right) \subset J_{F}(F)$,

(b) $T=J_{F}^{-1} \circ T^{\prime \prime} \circ J_{E}$, onde consideramos $J_{F}^{-1}: J_{F}(F) \longrightarrow F$. 


\subsection{Extensões de Arens}

Em 1951, R. Arens encontrou um modo de estender aplicações bilineares contínuas entre espaços de Banach para o bidual [Are51a, Are51b]. A seguir apresentaremos a construção das extensões de Arens.

Definição 4.2.1. Sejam $E_{1}, E_{2}, F$ espaços de Banach sobre $\mathbb{K}$. Dada uma aplicação bilinear continua $A: E_{1} \times E_{2} \longrightarrow F$, definimos $A^{t}: F^{\prime} \times E_{1} \longrightarrow E_{2}^{\prime}$ por:

$$
A^{t}\left(z^{\prime}, x\right)(y) \doteq z^{\prime}(A(x, y))
$$

para cada $z^{\prime} \in F^{\prime}, x \in E_{1}$ e $y \in E_{2}$. A aplicação $A^{t}$ é chamada de primeiro adjunto de A.

Proposição 4.2.2. A $A^{t}$ é uma aplicação bilinear contínua, com $\left\|A^{t}\right\| \leq\|A\|$.

Demonstração. Dados $\lambda \in \mathbb{K}, z_{1}^{\prime}, z_{2}^{\prime} \in F^{\prime}, x \in E_{1}$, para cada $y \in E_{2}$, temos:

$$
\begin{aligned}
A^{t}\left(\lambda z_{1}^{\prime}+z_{2}^{\prime}, x\right)(y) & \doteq\left(\lambda z_{1}^{\prime}+z_{2}^{\prime}\right)(A(x, y)) \\
& =\lambda z_{1}^{\prime}(A(x, y))+z_{2}^{\prime}(A(x, y)) \\
& =\lambda A^{t}\left(z_{1}^{\prime}, x\right)(y)+A^{t}\left(z_{2}^{\prime}, x\right)(y) \\
& =\left[\lambda A^{t}\left(z_{1}^{\prime}, x\right)+A^{t}\left(z_{2}^{\prime}, x\right)\right](y) .
\end{aligned}
$$

Dados $\lambda \in \mathbb{K}, z^{\prime} \in F^{\prime}, x_{1}, x_{2} \in E_{1}$, para cada $y \in E_{2}$, temos:

$$
\begin{aligned}
A^{t}\left(z^{\prime}, \lambda x_{1}+x_{2}\right)(y) & \doteq z^{\prime}\left(A\left(\lambda x_{1}+x_{2}, y\right)\right) \\
& =z^{\prime}\left(\lambda A\left(x_{1}, y\right)+A\left(x_{2}, y\right)\right) \\
& =\lambda z^{\prime}\left(A\left(x_{1}, y\right)\right)+z^{\prime}\left(A\left(x_{2}, y\right)\right) \\
& =\left[\lambda A^{t}\left(z^{\prime}, x_{1}\right)+A^{t}\left(z^{\prime}, x_{2}\right)\right](y) .
\end{aligned}
$$

Logo, $A^{t}$ é uma aplicação bilinear. Além disso, fixados $z^{\prime} \in F^{\prime}, x \in E_{1}$, para cada $y \in E_{2}$ :

$$
\left|A^{t}\left(z^{\prime}, x\right)(y)\right| \doteq \mid z^{\prime}\left(A(x, y) \mid \leq\left\|z^{\prime}\right\|\|A(x, y)\| \leq\left\|z^{\prime}\right\|\|A\|\|x\|\|y\|\right.
$$

Logo, $A^{t}\left(z^{\prime}, x\right) \in E_{2}^{\prime}$. Além disso, temos que:

$$
\left\|A^{t}\left(z^{\prime}, x\right)\right\|=\sup _{y \in B_{E_{2}}}\left|A^{t}\left(z^{\prime}, x\right)(y)\right| \leq\|A\|\left\|z^{\prime}\right\|\|x\|
$$

e, portanto, $A^{t}$ é contínua com $\left\|A^{t}\right\| \leq\|A\|$.

Analogamente ao que fizemos na Seção 4.1, a proposição anterior nos permite definir o segundo 
adjunto da aplicação bilinear $A: E_{1} \times E_{2} \longrightarrow F$ como $A^{t t} \doteq\left(A^{t}\right)^{t}$. Assim, $A^{t t}: E_{2}^{\prime \prime} \times F^{\prime} \longrightarrow E_{1}^{\prime}$ é dado por:

$$
A^{t t}\left(y^{\prime \prime}, z^{\prime}\right)(x) \doteq y^{\prime \prime}\left(A^{t}\left(z^{\prime}, x\right)\right), \quad \text { para cada } y^{\prime \prime} \in E_{2}^{\prime \prime}, z^{\prime} \in F^{\prime}, x \in E_{1} .
$$

Além disso, a proposição anterior nos diz também que $A^{t t}$ é bilinear contínuo com $\left\|A^{t t}\right\| \leq$ $\left\|A^{t}\right\| \leq\|A\|$. Procedendo da mesma maneira, definimos o terceiro adjunto de $A$ como $A^{t t t} \doteq\left(A^{t t}\right)^{t}$. Assim, $A^{t t t}: E_{1}^{\prime \prime} \times E_{2}^{\prime \prime} \longrightarrow F^{\prime \prime}$ é dado por:

$$
A^{t t t}\left(x^{\prime \prime}, y^{\prime \prime}\right)\left(z^{\prime}\right) \doteq x^{\prime \prime}\left(A^{t t}\left(y^{\prime \prime}, z^{\prime}\right)\right), \quad \text { para cada } \quad x^{\prime \prime} \in E_{1}^{\prime \prime}, y^{\prime \prime} \in E_{2}^{\prime \prime}, z^{\prime} \in F^{\prime}
$$

O terceiro adjunto de $A$ é bilinear contínuo e é tal que $\left\|A^{t t t}\right\| \leq\left\|A^{t t}\right\| \leq\left\|A^{t}\right\| \leq\|A\|$.

Teorema 4.2.3. Dada $A \in \mathscr{L}\left(E_{1}, E_{2} ; F\right)$, a aplicação $A^{t t t} \in \mathscr{L}\left(E_{1}^{\prime \prime}, E_{2}^{\prime \prime} ; F^{\prime \prime}\right)$ é uma extensão de $A$ no sentido de que $A^{\text {ttt }}\left(J_{E_{1}}(x), J_{E_{2}}(y)\right)=J_{F}(A(x, y))$, para todos $x \in E_{1}, y \in E_{2}$. Além disso, $\left\|A^{t t t}\right\|=\|A\|$.

Demonstração. Primeiro vejamos que o seguinte diagrama comuta:

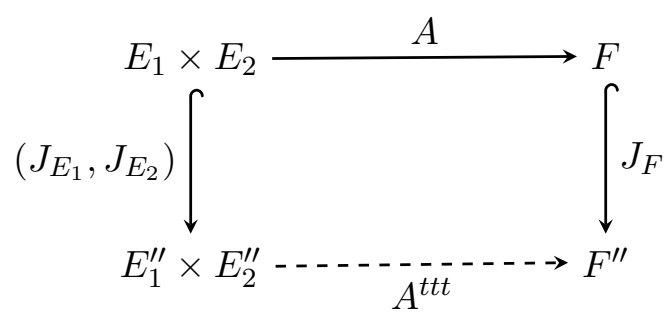

Dados $x \in E_{1}, y \in E_{2}$, para cada $z^{\prime} \in F^{\prime}$, temos:

$$
\begin{aligned}
A^{t t t}\left(J_{E_{1}}(x), J_{E_{2}}(y)\right)\left(z^{\prime}\right) & =A^{t t t}(\widehat{x}, \widehat{y})\left(z^{\prime}\right) \\
& \doteq \widehat{x}\left(A^{t t}\left(\widehat{y}, z^{\prime}\right)\right) \\
& =A^{t t}\left(\widehat{y}, z^{\prime}\right)(x) \\
& \doteq \widehat{y}\left(A^{t}\left(z^{\prime}, x\right)\right) \\
& =A^{t}\left(z^{\prime}, x\right)(y) \\
& \doteq z^{\prime}(A(x, y)) \\
& =J_{F}(A(x, y))\left(z^{\prime}\right) .
\end{aligned}
$$

Para mostrar que $\left\|A^{t t t}\right\|=\|A\|$, basta mostrar que vale $\|A\| \leq\left\|A^{t t t}\right\|$. De fato, como $A^{t t t}$ é uma extensão de $A$ e as inclusões canônicas $J_{E_{1}}, J_{E_{2}}$ e $J_{F}$ são imersões isométricas, para cada $(x, y) \in E_{1} \times E_{2}$, temos:

$$
\|A(x, y)\|=\left\|J_{F}(A(x, y))\right\|=\left\|A^{t t t}\left(J_{E_{1}}(x), J_{E_{2}}(y)\right)\right\| \leq\left\|A^{t t t}\right\|\left\|J_{E_{1}}(x)\right\|\left\|J_{E_{2}}(y)\right\|=\left\|A^{t t t}\right\|\|x\|\|y\| .
$$


Logo, $\|A\| \leq\left\|A^{t t t}\right\|$ e concluímos que a extensão preserva norma.

A aplicação $A^{t t t} \in \mathscr{L}\left(E_{1}^{\prime \prime}, E_{2}^{\prime \prime} ; F^{\prime \prime}\right)$ é dita a primeira extensão de Arens de $A$.

A partir de agora nos dedicaremos à construção de uma segunda extensão da aplicação multilinear $A \in \mathscr{L}\left(E_{1}, E_{2} ; F\right)$.

Definição 4.2.4. Sejam $E_{1}, E_{2}, F$ espaços de normados sobre um mesmo corpo $\mathbb{K}$. Dada $A \in$ $\mathscr{L}\left(E_{1}, E_{2} ; F\right)$, definimos a aplicação transposta de $A$ como $A^{T}: E_{2} \times E_{1} \longrightarrow F$, dada por:

$$
A^{T}(y, x) \doteq A(x, y) \quad \text { para cada } \quad x \in E_{1}, y \in E_{2} .
$$

Como a aplicação $A^{T}$ é bilinear e contínua, com $\|A\|=\left\|A^{T}\right\|$, segue do Teorema 4.2.3, que $A^{T t t t} \doteq\left(A^{T}\right)^{t t t}$ é uma extensão de $A^{T}$. Isto é, o seguinte diagrama comuta:

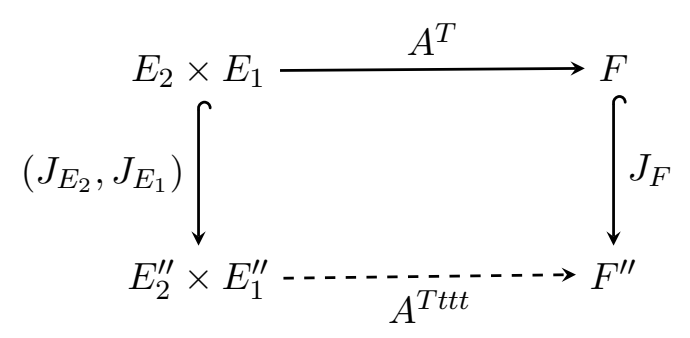

Logo, a aplicação $A^{T t t t T} \doteq\left(\left(A^{T}\right)^{t t t}\right)^{T}$ é uma extensão de $A$. De fato, dados $x \in E_{1}, y \in E_{2}$ :

$$
J_{F}(A(x, y))=J_{F}\left(A^{T}(y, x)\right)=A^{T t t t}\left(J_{E_{2}}(y), J_{E_{1}}(x)\right)=A^{T t t t T}\left(J_{E_{1}}(x), J_{E_{2}}(y)\right) .
$$

E segue que o seguinte diagrama comuta:

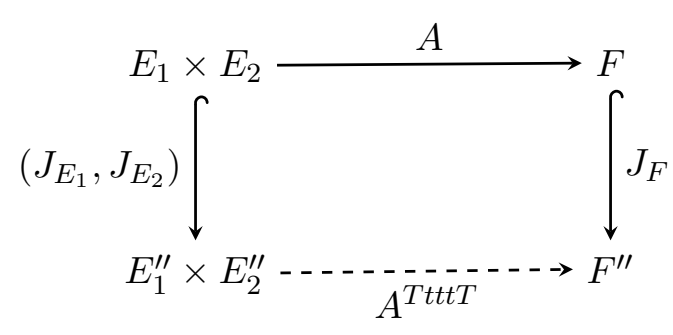

Além disso, decorre também do Teorema 4.2.3 que:

$$
\|A\|=\left\|A^{T}\right\|=\left\|A^{T t t t}\right\|=\left\|A^{T t t t T}\right\| .
$$

Assim, a aplicação $A^{T t t t T}$ será chamada de segunda extensão de Arens de $A$.

É necessário averiguar se essas duas construções nos levam a extensões distintas de uma mesma aplicação $A$ e se existem condições sob as quais essas extensões coincidam. Para isso, nos dedicaremos a estudar algumas propriedades dessa construção. 
Lema 4.2.5. Sejam $E_{1}, E_{2}, F$ espaços normados e $A \in \mathscr{L}\left(E_{1}, E_{2} ; F\right)$.

(a) $S e\left(x_{\alpha}\right) \subset E_{1}$ é um rede tal que $x_{\alpha} \stackrel{w^{*}}{\longrightarrow} x^{\prime \prime}$ em $E_{1}^{\prime \prime}$, então:

$$
A^{t t t}\left(x^{\prime \prime}, y^{\prime \prime}\right)=\lim _{\alpha} A^{t t t}\left(x_{\alpha}, y^{\prime \prime}\right) \text { em } F^{\prime \prime} \quad \text { para todo } y^{\prime \prime} \in E_{2}^{\prime \prime}
$$

(b) $S e\left(y_{\beta}\right) \subset E_{2}$ é um rede tal que $y_{\beta} \stackrel{w^{*}}{\longrightarrow} y^{\prime \prime}$ em $E_{2}^{\prime \prime}$, então:

$$
A^{T t t t T}\left(x^{\prime \prime}, y^{\prime \prime}\right)=\lim _{\beta} A^{T t t t T}\left(x^{\prime \prime}, y_{\beta}\right) \text { em } F^{\prime \prime} \quad \text { para todo } x^{\prime \prime} \in E_{1}^{\prime \prime} .
$$

Demonstração. (a): Primeiro suponha que $\left(x_{\alpha}\right) \subset E_{1}$ seja uma rede tal que $x_{\alpha} \stackrel{w^{*}}{\longrightarrow} 0$ em $E_{1}^{\prime \prime}$. Então, para cada $z^{\prime} \in F^{\prime}$ :

$$
\lim _{\alpha} A^{t t t}\left(x_{\alpha}, y^{\prime \prime}\right)\left(z^{\prime}\right)=\lim _{\alpha} x_{\alpha}(\underbrace{A^{t t}\left(y^{\prime \prime}, z^{\prime}\right)}_{\in E_{1}^{\prime}})=0
$$

Agora, se $\left(x_{\alpha}\right) \subset E_{1}$ é tal que $x_{\alpha} \stackrel{w^{*}}{\longrightarrow} x^{\prime \prime}$, então $x_{\alpha}-x^{\prime \prime} \stackrel{w^{*}}{\longrightarrow} 0$. Assim, para cada $z^{\prime} \in F^{\prime}$ :

$$
0=\lim _{\alpha} A^{t t t}\left(x_{\alpha}-x^{\prime \prime}, y^{\prime \prime}\right)\left(z^{\prime}\right)=\lim _{\alpha} A^{t t t}\left(x_{\alpha}, y^{\prime \prime}\right)\left(z^{\prime}\right)-A^{t t t}\left(x^{\prime \prime}, y^{\prime \prime}\right)\left(z^{\prime}\right)
$$

Portanto, $A^{t t t}\left(x^{\prime \prime}, y^{\prime \prime}\right)=\lim _{\alpha} A^{t t t}\left(x_{\alpha}, y^{\prime \prime}\right)$.

(b): Seja $\left(y_{\beta}\right) \subset E_{2} \operatorname{com} y_{\beta} \stackrel{w^{*}}{\longrightarrow} y^{\prime \prime}$ em $E_{2}^{\prime \prime}$. Então:

$$
\lim _{\beta} A^{T t t t T}\left(x^{\prime \prime}, y_{\beta}\right)=\lim _{\beta} A^{T t t t}\left(y_{\beta}, x^{\prime \prime}\right) \stackrel{(a)}{=} A^{T t t t}\left(y^{\prime \prime}, x^{\prime \prime}\right)=A^{T t t t T}\left(x^{\prime \prime}, y^{\prime \prime}\right)
$$

O próximo resultado nos mostra que as extensões de Arens coincidem quando pelo menos uma das entradas é avaliada sobre os pontos de $E_{1} \subset E_{1}^{\prime \prime}$ ou de $E_{2} \subset E_{2}^{\prime \prime}$.

Lema 4.2.6. Sejam $E_{1}, E_{2}, F$ espaços normados e $A \in \mathscr{L}\left(E_{1}, E_{2} ; F\right)$. Então:

(a) $A^{t t t}\left(x^{\prime \prime}, J_{E_{2}}(y)\right)=A^{T t t t T}\left(x^{\prime \prime}, J_{E_{2}}(y)\right)$ para cada $x^{\prime \prime} \in E_{1}^{\prime \prime}$ e $y \in E_{2}$.

(b) $A^{t t t}\left(J_{E_{1}}(x), y^{\prime \prime}\right)=A^{T t t t T}\left(J_{E_{1}}(x), y^{\prime \prime}\right)$ para cada $x \in E_{1}$ e $y^{\prime \prime} \in E_{2}^{\prime \prime}$.

Demonstração. (a): Para cada $z^{\prime} \in F^{\prime}$, temos:

$$
A^{t t t}\left(x^{\prime \prime}, J_{E_{2}}(y)\right)\left(z^{\prime}\right)=x^{\prime \prime}(\underbrace{A^{t t}\left(J_{E_{2}}(y), z^{\prime}\right)}_{\in E_{1}^{\prime}}) .
$$

Além disso, temos:

$$
\begin{aligned}
A^{T t t t T}\left(x^{\prime \prime}, J_{E_{2}}(y)\right)\left(z^{\prime}\right) & =A^{T t t t}\left(J_{E_{2}}(y), x^{\prime \prime}\right)\left(z^{\prime}\right) \\
& =J_{E_{2}}(y)\left(A^{T t t}\left(x^{\prime \prime}, z^{\prime}\right)\right)
\end{aligned}
$$




$$
\begin{aligned}
& =A^{T t t}\left(x^{\prime \prime}, z^{\prime}\right)(y) \\
& =x^{\prime \prime}(\underbrace{A^{T t}\left(z^{\prime}, y\right)}_{\in E_{1}^{\prime}}) .
\end{aligned}
$$

Basta mostrar que $A^{t t}\left(J_{E_{2}}(y), z^{\prime}\right)=A^{T t}\left(z^{\prime}, y\right)$. De fato, para cada $x \in E_{1}$, temos:

$$
\begin{aligned}
A^{t t}\left(J_{E_{2}}(y), z^{\prime}\right)(x) & =J_{E_{2}}(y)\left(A^{t}\left(z^{\prime}, x\right)\right) \\
& =A^{t}\left(z^{\prime}, x\right)(y) \\
& =z^{\prime}(A(x, y)) .
\end{aligned}
$$

Mas, por outro lado:

$$
\begin{aligned}
A^{T t}\left(z^{\prime}, y\right)(x) & =z^{\prime}\left(A^{T}(y, x)\right) \\
& =z^{\prime}(A(x, y)) .
\end{aligned}
$$

Logo, $A^{t t t}\left(x^{\prime \prime}, J_{E_{2}}(y)\right)=A^{T t t t T}\left(x^{\prime \prime}, J_{E_{2}}(y)\right)$ para cada $x^{\prime \prime} \in E_{1}^{\prime \prime}$ e $y \in E_{2}$.

(b): Para cada $z^{\prime} \in F^{\prime}$, temos:

$$
\begin{aligned}
A^{t t t}\left(J_{E_{1}}(x), y^{\prime \prime}\right)\left(z^{\prime}\right) & =J_{E_{1}}(x)\left(A^{t t}\left(y^{\prime \prime}, z^{\prime}\right)\right. \\
& =A^{t t}\left(y^{\prime \prime}, z^{\prime}\right)(x) \\
& =y^{\prime \prime}(\underbrace{A^{t}\left(z^{\prime}, x\right)}_{\in E_{2}^{\prime}}) .
\end{aligned}
$$

Além disso, temos:

$$
\begin{aligned}
A^{T t t t T}\left(J_{E_{1}}(x), y^{\prime \prime}\right)\left(z^{\prime}\right) & =A^{T t t t}\left(y^{\prime \prime}, J_{E_{1}}(x)\right)\left(z^{\prime}\right) \\
& =y^{\prime \prime}(\underbrace{A^{T t t}\left(J_{E_{1}}(x), z^{\prime}\right)}_{\in E_{2}^{\prime}}) .
\end{aligned}
$$

Basta mostrar que $A^{t}\left(z^{\prime}, x\right)=A^{T t t}\left(J_{E_{1}}(x), z^{\prime}\right)$. De fato, para cada $y \in E_{2}$ :

$$
\begin{aligned}
A^{T t t}\left(J_{E_{1}}(x), z^{\prime}\right)(y) & =J_{E_{1}}(x)\left(A^{T t}\left(z^{\prime}, y\right)\right) \\
& =A^{T t}\left(z^{\prime}, y\right)(x) \\
& =z^{\prime}\left(A^{T}(y, x)\right) \\
& =z^{\prime}(A(x, y)) \\
& =A^{t}\left(z^{\prime}, x\right)(y)
\end{aligned}
$$

Portanto, $A^{t t t}\left(J_{E_{1}}(x), y^{\prime \prime}\right)=A^{T t t t T}\left(J_{E_{1}}(x), y^{\prime \prime}\right)$ para cada $x \in E_{1}$ e $y^{\prime \prime} \in E_{2}^{\prime \prime}$. 
Teorema 4.2.7. Sejam $E_{1}, E_{2}$ espaços normados e $A \in \mathscr{L}\left(E_{1}, E_{2} ; F\right)$. Se $x^{\prime \prime} \in E_{1}^{\prime \prime}, y^{\prime \prime} \in E_{2}^{\prime \prime}$, dadas redes $\left(x_{\alpha}\right) \subset E_{1},\left(y_{\beta}\right) \subset E_{2}$ tais que $x_{\alpha} \stackrel{w^{*}}{\longrightarrow} x^{\prime \prime}$ e $y_{\beta} \stackrel{w^{*}}{\longrightarrow} y^{\prime \prime}$, então:

(a) $A^{t t t}\left(x^{\prime \prime}, y^{\prime \prime}\right)=\lim _{\alpha} \lim _{\beta} A\left(x_{\alpha}, y_{\beta}\right)$

(b) $A^{T t t t T}\left(x^{\prime \prime}, y^{\prime \prime}\right)=\lim _{\beta} \lim _{\alpha} A\left(x_{\alpha}, y_{\beta}\right)$

Demonstração. Como $A^{t t t}$ estende $A$, vale que $A\left(x_{\alpha}, y_{\beta}\right)=A^{t t t}\left(x_{\alpha}, y_{\beta}\right)$. Pelo Lema 4.2 .5 e pelo Lema 4.2.6, temos:

$$
\begin{aligned}
\lim _{\alpha} \lim _{\beta} A\left(x_{\alpha}, y_{\beta}\right) & =\lim _{\alpha} \lim _{\beta} A^{t t t}\left(x_{\alpha}, y_{\beta}\right) \\
& =\lim _{\alpha} \lim _{\beta} A^{T t t t T}\left(x_{\alpha}, y_{\beta}\right) \\
& =\lim _{\alpha} A^{T t t t T}\left(x_{\alpha}, y^{\prime \prime}\right) \\
& =\lim _{\alpha} A^{t t t}\left(x_{\alpha}, y^{\prime \prime}\right) \\
& =A^{t t t}\left(x^{\prime \prime}, y^{\prime \prime}\right)
\end{aligned}
$$

Com argumento análogo, segue que $A^{T t t t T}\left(x^{\prime \prime}, y^{\prime \prime}\right)=\lim _{\beta} \lim _{\alpha} A\left(x_{\alpha}, y_{\beta}\right)$.

A partir do Lema 4.2.5, do Lema 4.2.6 e do Teorema 4.2.7, apresentamos a seguinte caracterização:

Teorema 4.2.8. Sejam $E_{1}, E_{2}, F$ espaços normados e $A \in \mathscr{L}\left(E_{1} \times E_{2}, F\right)$. São equivalentes:

(a) $A^{t t t}=A^{T t t t T}$

(b) Para cada $x^{\prime \prime} \in E_{1}^{\prime \prime}, y^{\prime \prime} \in E_{2}^{\prime \prime}$, existem redes $\left(x_{\alpha}\right) \subset E_{1}$ e $\left(y_{\beta}\right) \subset E_{2}$ com $x_{\alpha} \stackrel{w^{*}}{\longrightarrow} x^{\prime \prime}$ e $y_{\beta} \stackrel{w^{*}}{\longrightarrow} y^{\prime \prime}$ tais que:

$$
\lim _{\alpha} \lim _{\beta} A\left(x_{\alpha}, y_{\beta}\right)=\lim _{\beta} \lim _{\alpha} A\left(x_{\alpha}, y_{\beta}\right)
$$

Em geral, $A^{t t t} \neq A^{T t t t T}$. O seguinte exemplo mostra que faz parte do artigo [AGM03].

Exemplo 4.2.9. Ao longo deste exemplo denotaremos $A^{t t t}=A_{12}$ e $A^{T t t t T}=A_{21}$ para indicar a ordem dos limites iterados do Teorema 4.2.7.

Seja $A: l_{1} \times l_{1} \longrightarrow \mathbb{K}$ definida por:

$$
A(x, y) \doteq \sum_{n=1}^{\infty} x_{n}\left(\sum_{k=1}^{n} \frac{k}{k+1} y_{k}\right) \text { para cada } x, y \in l_{1} \text {, onde } x=\left(x_{n}\right) \text { e } y=\left(y_{n}\right)
$$

Fixados $x, y \in l_{1}$, para cada $N \in \mathbb{N}$, temos:

$$
\sum_{n=1}^{N}\left|x_{n}\left(\sum_{k=1}^{n} \frac{k}{k+1} y_{k}\right)\right| \leq \sum_{n=1}^{N}\left|x_{n}\right|\left(\sum_{k=1}^{n}\left|y_{k}\right|\right) \leq\|x\|\|y\|
$$


Logo, $A(x, y)$ é uma série absolutamente convergente e, portanto, a aplicação A está bem definida.

Dados $x, y, z \in l_{1}$ e $\lambda \in \mathbb{K}$, temos:

$$
\begin{aligned}
A(x, \lambda y+z) & =\sum_{n=1}^{\infty} x_{n}\left(\sum_{k=1}^{n} \frac{k}{k+1}\left(\lambda y_{k}+z_{k}\right)\right) \\
& =\lambda \sum_{n=1}^{\infty} x_{n}\left(\sum_{k=1}^{n} \frac{k}{k+1} y_{k}\right)+\sum_{n=1}^{\infty} x_{n}\left(\sum_{k=1}^{n} \frac{k}{k+1} z_{k}\right) \\
& =\lambda A(x, y)+A(x, z) . \\
A(\lambda x+y, z) & =\sum_{n=1}^{\infty}\left(\lambda x_{n}+y_{n}\right)\left(\sum_{k=1}^{n} \frac{k}{k+1} z_{k}\right) \\
& =\sum_{n=1}^{\infty}\left[\lambda x_{n}\left(\sum_{k=1}^{n} \frac{k}{k+1} z_{k}\right)+y_{n}\left(\sum_{k=1}^{n} \frac{k}{k+1} z_{k}\right)\right] \\
& =\sum_{n=1}^{\infty} \lambda x_{n}\left(\sum_{k=1}^{n} \frac{k}{k+1} z_{k}\right)+\sum_{n=1}^{\infty} y_{n}\left(\sum_{k=1}^{n} \frac{k}{k+1} z_{k}\right) \\
& =\lambda A(x, z)+A(y, z) .
\end{aligned}
$$

Segue que a aplicação $A$ é bilinear e, por (4.1), $\|A\| \leq 1$. Denote por $\left(e_{n}\right)$ a base de Schauder canônica de $l_{1}$. Para cada $j \in \mathbb{N}$ :

$$
\left|A\left(e_{j}, e_{j}\right)\right|=\sum_{n=1}^{\infty} \delta_{n j}\left(\sum_{k=1}^{n} \frac{k}{k+1} \delta_{k j}\right)=\frac{j}{j+1} .
$$

Logo, tomando supremo sobre $B_{l_{1} \times l_{1}}$, segue que $\|A\| \geq 1$ e, portanto, $\|A\|=1$.

Note que, como $B_{l_{1}^{\prime \prime}}$ é $w^{*}$-compacta, existem $z \in B_{l_{1}^{\prime \prime}}$ e uma subrede de $\left(e_{n}\right)$ que é $w^{*}$-convergente $\grave{a} z$. Afirmamos que se $z \in l_{1}^{\prime \prime}$ é ponto de $w^{*}$-acumulação de $\left(e_{n}\right)$, então $1=A_{21}(z, z)=\left\|A_{21}\right\|$. Pelo Teorema 4.2.7, denotando por $\left(e_{\alpha}\right)_{\alpha \in \Lambda} e\left(e_{\beta}\right)_{\beta \in \Gamma}$ as subredes convergentes à $z$ :

$$
A_{21}(z, z)=\lim _{\beta} \lim _{\alpha} A\left(e_{\alpha}, e_{\beta}\right)
$$

Mas, para $j, k \in \mathbb{N}$, temos:

$$
\lim _{j \in \mathbb{N}} A\left(e_{j}, e_{k}\right)=\lim _{j \in \mathbb{N}} \sum_{n=1}^{\infty} \delta_{n j}\left(\sum_{i=1}^{n} \frac{i}{i+1} \delta_{i k}\right)=\frac{k}{k+1} \stackrel{k \rightarrow \infty}{\longrightarrow} 1 .
$$

Como o limite de qualquer subrede de $\left(e_{n}\right)_{n \in \mathbb{N}}$ deve ser o mesmo, $A_{21}(z, z)=1=\|A\|=\left\|A_{21}\right\|$ e a afirmação está provada.

Para provar que $A_{12} \neq A_{21}$, suponha que existam $z, w \in B_{l_{1}^{\prime \prime}}$ tais que $A_{12}(z, w)=1$. Pelo 
Teorema de Goldstine, existe $\left(y_{\beta}\right) \subset B_{l_{1}}$ tal que $y_{\beta} \stackrel{w^{*}}{\longrightarrow} w$, onde $y_{\beta}=\left(y_{k, \beta}\right)_{k \in \mathbb{N}}$.

Afirmamos que, para cada $k \in \mathbb{N}, y_{k, \beta} \stackrel{\beta}{\longrightarrow} 0$. Vejamos que isso, de fato, ocorre.

Suponha, por absurdo, que existam $k_{0} \in \mathbb{N} e 0<\delta<1$ tais que $\left|y_{k, \beta}\right|>\delta$ para alguma subrede de $\left(y_{\beta}\right)$. Se $x=\left(x_{n}\right) \in B_{l_{1}}$, então:

$$
\left|A_{12}(x, w)\right|=\left|\lim _{\beta} A\left(x, y_{\beta}\right)\right|=\lim _{\beta}\left|A\left(x, y_{\beta}\right)\right|
$$

Além disso, temos:

$$
\begin{aligned}
\left|A\left(x, y_{\beta}\right)\right| & \leq \sum_{n=1}^{\infty}\left|x_{n}\right|\left(\sum_{k=1}^{n} \frac{k}{k+1}\left|y_{k, \beta}\right|\right) \\
& \leq \sum_{n=1}^{\infty}\left|x_{n}\right|\left(\frac{k_{0}}{k_{0}+1}\left|y_{k_{0}, \beta}\right|+\sum_{\substack{k=1 \\
k \neq k_{0}}}^{\infty} \frac{k}{k+1}\left|y_{k, \beta}\right|\right) \\
& \leq \sum_{n=1}^{\infty}\left|x_{n}\right|\left(\frac{k_{0}}{k_{0}+1}\left|y_{k_{0}, \beta}\right|+\sum_{\substack{k=1 \\
k \neq k_{0}}}^{\infty}\left|y_{k, \beta}\right|\right) \\
& \leq \sum_{n=1}^{\infty}\left|x_{n}\right|\left(\frac{k_{0}}{k_{0}+1}\left|y_{k_{0}, \beta}\right|+\left(1-\left|y_{k_{0}, \beta}\right|\right)\right) \\
& \leq 1-\left|y_{k_{0}, \beta}\right|+\frac{k_{0}}{k_{0}+1}\left|y_{k_{0}, \beta}\right| \\
& =1-\underbrace{\delta\left[1-\frac{k_{0}}{k_{0}+1}\right]}_{C} .
\end{aligned}
$$

Logo, se $\left(x_{\alpha}\right) \subset B_{l_{1}}$ é tal que $x_{\alpha} \stackrel{w^{*}}{\longrightarrow} z$, então:

$$
1=A_{12}(z, w)=\lim _{\alpha} \lim _{\beta}\left|A\left(x_{\alpha}, y_{\beta}\right)\right| \leq 1-C<1
$$

uma contradição. Portanto $y_{k, \beta} \stackrel{\beta}{\longrightarrow} 0$ para todo $k \in \mathbb{N}$.

Agora, dados $x \in B_{l_{1}}$ e $\varepsilon>0$, existe $n_{0} \in \mathbb{N}$ tal que $\sum_{n>n_{0}}\left|x_{n}\right|<\frac{\varepsilon}{2}$. Além disso, existe $\beta_{0}$ tal que, para todo $\beta \geq \beta_{0}$ :

$$
\sum_{k=1}^{n_{0}} \frac{k}{k+1}\left|y_{k, \beta}\right| \leq \frac{\varepsilon}{2} .
$$

Desta maneira, se $\beta \geq \beta_{0}$, temos:

$$
\left|A\left(x, y_{\beta}\right)\right| \leq \underbrace{\sum_{n=1}^{n_{0}}\left|x_{n}\right|}_{\leq\|x\| \leq 1}\left(\sum_{k=1}^{n} \frac{k}{k+1}\left|y_{k, \beta}\right|\right)+\sum_{n>n_{0}}\left|x_{n}\right| \underbrace{\left(\sum_{k=1}^{n} \frac{k}{k+1}\left|y_{k, \beta}\right|\right)}_{\leq\left\|y_{\beta}\right\| \leq 1}
$$




$$
\begin{aligned}
& \leq \sum_{k=1}^{n} \frac{k}{k+1}\left|y_{k, \beta}\right|+\sum_{n>n_{0}}\left|x_{n}\right| \\
& <\varepsilon .
\end{aligned}
$$

Logo, $A(x, w)=\lim _{\beta} A\left(x, y_{\beta}\right)=0$ para todo $x \in B_{l_{1}}$. Em particular, temos:

$$
1=A_{12}(z, w)=\lim _{\alpha} \lim _{\beta} A\left(x_{\alpha}, y_{\beta}\right)=0
$$

uma contradição.

Tomando $z \in B_{l_{1}^{\prime \prime}}$ ponto de $w^{*}$-acumulação de $\left(e_{n}\right)$, temos:

$$
A_{21}(z, z)=1 \quad e \quad A_{12}(z, z) \neq 1 .
$$

Estamos interessados em estender polinômios homogêneos, e estes se relacionam naturalmente com as aplicações simétricas. Vamos estudar algumas consequências da hipótese de simetria sobre a aplicação $A: E^{2} \longrightarrow F$, bem como o comportamento das extensões de Arens quando calculadas na diagonal de $\left(E^{\prime \prime}\right)^{2}$.

Proposição 4.2.10. Se $A \in \mathscr{L}^{s}\left(E^{2}, F\right)$ e $A^{t t t}=A^{T t t t T}$, então $A^{t t t} \in \mathscr{L}^{s}\left(\left(E^{\prime \prime}\right)^{2}, F\right)$.

Demonstração. Basta notar que, como $A$ é simétrica:

$$
A^{t t t}=A^{T t t t T}=\left(A^{T}\right)^{t t t T}=A^{t t t T}=\left(A^{t t t}\right)^{T},
$$

ou seja, $A^{t t t}$ é simétrica.

Proposição 4.2.11. Seja $A \in \mathscr{L}^{s}\left(E^{2}, F\right)$. Se $A^{t t t}$ é simétrica, então $A^{t t t}=A^{T t t t T}$. Demonstração. Observe que:

$$
A^{t t t}=\left(A^{t t t}\right)^{T}=(A)^{t t t T}=\left(A^{T}\right)^{t t t T}=A^{T t t t T},
$$

ou seja, as extensões coincidem.

Proposição 4.2.12. Sejam $A \in \mathscr{L}^{s}\left({ }^{2} E, F\right)$ e $x^{\prime \prime} \in E^{\prime \prime}$, então:

$$
A^{t t t}\left(x^{\prime \prime}, x^{\prime \prime}\right)=A^{T t t t T}\left(x^{\prime \prime}, x^{\prime \prime}\right)
$$

Demonstração. Se $\left(x_{\alpha}\right),\left(x_{\beta}\right) \subset E$ são redes tais que $x_{\alpha} \stackrel{w^{*}}{\longrightarrow} x^{\prime \prime}$ e $x_{\beta} \stackrel{w^{*}}{\longrightarrow} x^{\prime \prime}$, então: 


$$
\begin{aligned}
A^{t t t}\left(x^{\prime \prime}, x^{\prime \prime}\right) & =\lim _{\alpha} \lim _{\beta} A\left(x_{\alpha}, x_{\beta}\right) & & \text { (Teorema 4.2.7) } \\
& =\lim _{\alpha} \lim _{\beta} A\left(x_{\beta}, x_{\alpha}\right) & & (A \text { é simétrica) } \\
& =\lim _{\alpha} \lim _{\beta} A^{t t t}\left(x_{\beta}, x_{\alpha}\right) & & \left(A^{t t t} \text { é extensão }\right) \\
& =\lim _{\alpha} A^{t t t}\left(x^{\prime \prime}, x_{\alpha}\right) & & (\text { Lema 4.2.5) } \\
& =\lim _{\alpha} A^{T t t t T}\left(x^{\prime \prime}, x_{\alpha}\right) & & (\text { Lema 4.2.6) } \\
& =A^{T t t t T}\left(x^{\prime \prime}, x^{\prime \prime}\right) . & & \text { (Lema 4.2.5) }
\end{aligned}
$$

A partir da proposição anterior, podemos definir a extensão de um polinômio 2-homogêneo da seguinte maneira:

Definição 4.2.13. Sejam $P \in \mathscr{P}\left({ }^{2} E, F\right)$ e $A \in \mathscr{L}^{s}\left({ }^{2} E, F\right)$ sua bilinear simétrica associada. A extensão de $P$ ao bidual $E^{\prime \prime}$ é a função $\bar{P}: E^{\prime \prime} \longrightarrow F^{\prime \prime}$, dada por:

$$
\bar{P}\left(x^{\prime \prime}\right) \doteq A^{t t t}\left(x^{\prime \prime}, x^{\prime \prime}\right)=A^{T t t t T}\left(x^{\prime \prime}, x^{\prime \prime}\right) \quad \text { para todo } x^{\prime \prime} \in E^{\prime \prime} .
$$

A definição acima nos dá idéia de como deve ser a definição geral da extensão de polinômios $m$-homogêneos ao bidual e, deste modo, nos motiva a estudar extensões de aplicações multilineares. Abordaremos estes temas ao longo das próximas seções.

\subsection{Construção de Aron-Berner}

A seguir apresentaremos a construção de Richard M. Aron e Paul D. Berner para estender aplicações multilineares com imagem num corpo $\mathbb{K}$, este método está presente em [AB78] e pode ser adaptado para aplicações multilineares à valores vetoriais (veja [Din12] pág. 413). Analogamente ao que fizemos na Seção 4.2 , dada uma aplicação $m$-linear contínua $A \in \mathscr{L}\left(E_{1}, \ldots, E_{m}\right)$, queremos obter uma aplicação $\bar{A} \in \mathscr{L}\left(E_{1}^{\prime \prime}, \ldots, E_{m}^{\prime \prime}\right)$ tal que o seguinte diagrama comute:

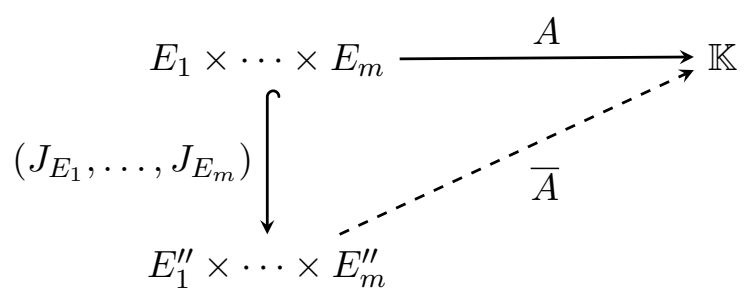

Isto é, que $\bar{A}\left(J_{E_{1}}\left(x_{1}\right), \ldots, J_{E_{m}}\left(x_{m}\right)\right)=A\left(x_{1}, \ldots, x_{m}\right)$ para cada $\left(x_{1}, \ldots, x_{m}\right) \in E_{1} \times \cdots \times E_{m}$. Inicialmente apresentaremos a construção para o caso $m=3$ para que o leitor fique familiarizado com a notação e, posteriormente, apresentaremos o caso geral. 
Sejam $E_{1}, E_{2}, E_{3}$ espaços de Banach sobre um mesmo corpo $\mathbb{K}$ e $A \in \mathscr{L}\left(E_{1}, E_{2}, E_{3}\right)$. Fixado $z_{3} \in E_{3}^{\prime \prime}$, defina a função $\overline{z_{3}}: \mathscr{L}\left(E_{1}, E_{2}, E_{3}\right) \longrightarrow \mathscr{L}\left(E_{1}, E_{2}\right)$ da seguinte maneira:

$$
\overline{z_{3}}(M)\left(x_{1}, x_{2}\right) \doteq z_{3}\left(M\left(x_{1}, x_{2}, \cdot\right)\right) \quad \text { para cada } M \in \mathscr{L}\left(E_{1}, E_{2}, E_{3}\right),
$$

onde $M\left(x_{1}, x_{2}, \cdot\right): E_{3} \longrightarrow \mathbb{K}$ é o funcional linear e contínuo dado por:

$$
M\left(x_{1}, x_{2}, \cdot\right)\left(x_{3}\right) \doteq M\left(x_{1}, x_{2}, x_{3}\right) .
$$

Assim, $\overline{z_{3}}$ é linear e contínua, com $\left\|\overline{z_{3}}\right\| \leq\left\|z_{3}\right\|$. De fato, dados $M_{1}, M_{2} \in \mathscr{L}\left(E_{1}, E_{2}, E_{3}\right), \lambda \in \mathbb{K}$, para cada $\left(x_{1}, x_{2}\right) \in E_{1} \times E_{2}$ :

$$
\begin{aligned}
\overline{z_{3}}\left(\lambda M_{1}+M_{2}\right)\left(x_{1}, x_{2}\right) & \doteq z_{3}\left(\left(\lambda M_{1}+M_{2}\right)\left(x_{1}, x_{2}, \cdot\right)\right) \\
& =z_{3}\left(\lambda M_{1}\left(x_{1}, x_{2}, \cdot\right)+M_{2}\left(x_{1}, x_{2}, \cdot\right)\right) \\
& =\lambda z_{3}\left(M_{1}\left(x_{1}, x_{2}, \cdot\right)\right)+z_{3}\left(M_{2}\left(x_{1}, x_{2}, \cdot\right)\right) \\
& =\left[\lambda \overline{z_{3}}\left(M_{1}\right)+\overline{z_{3}}\left(M_{2}\right)\right]\left(x_{1}, x_{2}\right) .
\end{aligned}
$$

Além disso:

$$
\begin{aligned}
\left\|\overline{z_{3}}\right\| & =\sup _{\|M\| \leq 1}\left\|\overline{z_{3}}(M)\right\| \\
& =\sup _{\|M\| \leq 1}\left(\sup _{\substack{\left\|x_{1}\right\| \leq 1 \\
\left\|x_{2}\right\| \leq 1}}\left|\overline{z_{3}}(M)\left(x_{1}, x_{2}\right)\right|\right) \\
& =\sup _{\|M\| \leq 1}\left(\sup _{\substack{\left\|x_{1}\right\| \leq 1 \\
\left\|x_{2}\right\| \leq 1}}\left|z_{3}\left(M\left(x_{1}, x_{2}, \cdot\right)\right)\right|\right) \\
& \leq \sup _{\|M\| \leq 1}\left(\sup _{\substack{\left\|x_{1}\right\| \leq 1 \\
\left\|x_{2}\right\| \leq 1}}\left\|z_{3}\right\|\left\|M\left(x_{1}, x_{2}, \cdot\right)\right\|\right) \\
& =\left\|z_{3}\right\| \sup _{\|M\| \leq 1}\left(\sup _{\substack{\left\|x_{1}\right\| \leq 1 \\
\left\|x_{2}\right\| \leq 1}}\left(\sup _{\left\|x_{3}\right\| \leq 1}\left|M\left(x_{1}, x_{2}, x_{3}\right)\right|\right)\right) \\
& \leq\left\|z_{3}\right\| \sup _{\|M\| \leq 1}\left(\sup _{\substack{\left\|x_{1}\right\| \leq 1 \\
\left\|x_{2}\right\| \leq 1}}\left(\sup _{\left\|x_{3}\right\| \leq 1}\|M\|\left\|x_{1}\right\|\left\|x_{2}\right\|\left\|x_{3}\right\|\right)\right) \\
& \leq\left\|z_{3}\right\| .
\end{aligned}
$$

Agora, fixado $z_{2} \in E_{2}^{\prime \prime}$, definimos $\overline{z_{2}}: \mathscr{L}\left(E_{1}, E_{2}\right) \longrightarrow \mathscr{L}\left(E_{1}\right)=E_{1}^{\prime}$ por: 


$$
\overline{z_{2}}(M)\left(x_{1}\right) \doteq z_{2}\left(M\left(x_{1}, \cdot\right)\right) \quad \text { para cada } M \in \mathscr{L}\left(E_{1}, E_{2}\right)
$$

onde $M\left(x_{1}, \cdot\right): E_{2} \longrightarrow \mathbb{K}$ é o funcional linear e contínuo dado por:

$$
M\left(x_{1}, \cdot\right)\left(x_{2}\right) \doteq M\left(x_{1}, x_{2}\right)
$$

Desta maneira, analogamente ao que fizemos, segue que $\overline{z_{2}}$ é contínuo e $\left\|\overline{z_{2}}\right\| \leq\left\|z_{2}\right\|$.

Por último, dado $z_{1} \in E_{1}^{\prime \prime}$, definimos $\overline{z_{1}}: \mathscr{L}\left(E_{1}\right)=E_{1}^{\prime} \longrightarrow \mathbb{K}$ por $\overline{z_{1}} \doteq z_{1}$. Com isso, obtemos a seguinte cadeia:

$$
\mathscr{L}\left(E_{1}, E_{2}, E_{3}\right) \stackrel{\overline{z_{3}}}{\longrightarrow} \mathscr{L}\left(E_{1}, E_{2}\right) \stackrel{\overline{z_{2}}}{\longrightarrow} \mathscr{L}\left(E_{1}\right)=E_{1}^{\prime} \stackrel{\overline{z_{1}}}{\longrightarrow} \mathbb{K}
$$

Além disso, observe que dados $\lambda \in \mathbb{K}$ e $z_{i}^{(1)}, z_{i}^{(2)} \in E_{i}^{\prime \prime}$ para $1 \leq i \leq 3$, vale:

$$
\overline{\lambda z_{i}^{(1)}+z_{i}^{(2)}}=\lambda \overline{z_{i}^{(1)}}+\overline{z_{i}^{(2)}} .
$$

Para o caso $i=3$, dados $M \in \mathscr{L}\left(E_{1}, E_{2}, E_{3}\right)$ e $\left(x_{1}, x_{2}\right) \in E_{1} \times E_{2}$ :

$$
\begin{aligned}
\overline{\lambda z_{3}^{(1)}+z_{3}^{(2)}}(M)\left(x_{1}, x_{2}\right) & \doteq\left(\lambda z_{3}^{(1)}+z_{3}^{(2)}\right)\left(M\left(x_{1}, x_{2}, \cdot\right)\right) \\
& =\lambda z_{3}^{(1)}\left(M\left(x_{1}, x_{2}, \cdot\right)\right)+z_{3}^{(2)}\left(M\left(x_{1}, x_{2}, \cdot\right)\right) \\
& =\left[\lambda \overline{z_{3}^{(1)}}+\overline{z_{3}^{(2)}}\right](M)\left(x_{1}, x_{2}\right) .
\end{aligned}
$$

Para o caso $i=2$, dados $M \in \mathscr{L}\left(E_{1}, E_{2}\right)$ e $x_{1} \in E_{1}$ :

$$
\begin{aligned}
\overline{\lambda z_{2}^{(1)}+z_{2}^{(2)}}(M)\left(x_{1}\right) & \doteq\left(\lambda z_{2}^{(1)}+z_{2}^{(2)}\right)\left(M\left(x_{1}, \cdot\right)\right) \\
& =\lambda z_{2}^{(1)}\left(M\left(x_{1}, \bullet\right)\right)+z_{2}^{(2)}\left(M\left(x_{1}, \cdot\right)\right) \\
& =\left[\lambda \overline{z_{2}^{(1)}}+\overline{z_{2}^{(2)}}\right](M)\left(x_{1}\right) .
\end{aligned}
$$

Para o caso $i=1$, dado $M \in \mathscr{L}\left(E_{1}\right)=E_{1}^{\prime}$ :

$$
\overline{\lambda z_{1}^{(1)}+z_{1}^{(2)}}(M) \doteq\left(\lambda z_{1}^{(1)}+z_{1}^{(2)}\right)(M)=\left[\lambda \overline{z_{1}^{(1)}}+\overline{z_{1}^{(2)}}\right](M) .
$$

A partir da cadeia acima e de $A \in \mathscr{L}\left(E_{1}, E_{2}, E_{3}\right)$, estamos em condições de definir uma aplicação $\bar{A}: E_{1}^{\prime \prime} \times E_{2}^{\prime \prime} \times E_{3}^{\prime \prime} \longrightarrow \mathbb{K}$ da seguinte maneira:

$$
\bar{A}\left(z_{1}, z_{2}, z_{3}\right) \doteq \overline{z_{1}} \circ \overline{z_{2}} \circ \overline{z_{3}}(A) \text { para cada } z_{1} \in E_{1}^{\prime \prime}, z_{2} \in E_{2}^{\prime \prime}, z_{3} \in E_{3}^{\prime \prime} .
$$

Segue da propriedade (1) que a aplicação $\bar{A}$ é linear em cada uma de suas entradas. Além disso, 
para cada $z_{1} \in E_{1}^{\prime \prime}, z_{2} \in E_{2}^{\prime \prime}, z_{3} \in E_{3}^{\prime \prime}$, temos:

$$
\left\|\bar{A}\left(z_{1}, z_{2}, z_{3}\right)\right\|=\left|\overline{z_{1}} \circ \overline{z_{2}} \circ \overline{z_{3}}(A)\right| \leq\left\|\overline{z_{1}}\right\|\left\|\overline{z_{2}}\right\|\left\|\overline{z_{3}}\right\|\|A\| \leq\|A\|\left\|z_{1}\right\|\left\|z_{2}\right\|\left\|z_{3}\right\| .
$$

Isto é, $\bar{A} \in \mathscr{L}\left(E_{1}^{\prime \prime}, E_{2}^{\prime \prime}, E_{3}^{\prime \prime}\right)$ e $\|\bar{A}\| \leq\|A\|$.

Além disso, para cada $x_{1} \in E_{1}, x_{2} \in E_{2}, x_{3} \in E_{3}$, temos:

$$
\begin{aligned}
\bar{A}\left(J_{E_{1}}\left(x_{1}\right), J_{E_{2}}\left(x_{2}\right), J_{E_{3}}\left(x_{3}\right)\right) & \doteq \overline{J_{E_{1}}\left(x_{1}\right)} \circ \overline{J_{E_{2}}\left(x_{2}\right)} \circ \overline{J_{E_{3}}\left(x_{3}\right)}(A) \\
& =\overline{J_{E_{1}}\left(x_{1}\right)} \underbrace{\left.\overline{J_{E_{2}}\left(x_{2}\right)} \circ \overline{J_{E_{3}}\left(x_{3}\right)}(A)\right)}_{\epsilon E_{1}^{\prime}=\mathscr{L}\left(E_{1}\right)} \\
& \left.\doteq \overline{J_{E_{2}}\left(x_{2}\right)} \circ \overline{J_{E_{3}}\left(x_{3}\right)}(A)\right)\left(x_{1}\right) \\
& =\overline{J_{E_{2}}\left(x_{2}\right)} \underbrace{\left(\overline{J_{E_{3}}\left(x_{3}\right)}(A)\right)}\left(x_{1}\right) \\
& \doteq J_{E_{2}\left(x_{2}\right)}\left(\overline{J_{E_{3}}\left(x_{3}\right)}(A)\left(x_{1}, \cdot\right)\right) \\
& =\overline{J_{E_{3}}\left(x_{3}\right)}(A)\left(x_{1}, \cdot\right)\left(x_{2}\right) \\
& =\overline{J_{E_{3}}\left(x_{3}\right)}(A)\left(x_{1}, x_{2}\right) \\
& \doteq \overline{J_{E_{3}}\left(x_{3}\right)}\left(A\left(x_{1}, x_{2}, \cdot\right)\right) \\
& =A\left(x_{1}, x_{2}, x_{3}\right) .
\end{aligned}
$$

Acabamos de provar que $\bar{A}$ é uma extensão de $A$. Além disso, também provamos que $\|\bar{A}\|=\|A\|$ pois o seguinte diagrama comuta:

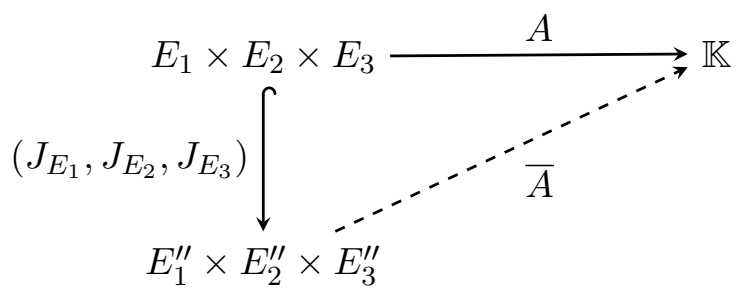

Deste modo, $\bar{A}$ será chamada de extensão de Aron-Berner de $A$.

Observação 4.3.1. Note que $\bar{A}$ foi obtida estendendo primeiramente a última entrada de A. Entretanto, poderíamos começar esta construção escolhendo qualquer uma das variáveis. Para exemplificar este fato, começaremos a construção estendendo a segunda variável e, em seguida, estenderemos a primeira:

Considere $A_{1}: E_{1} \times E_{2}^{\prime \prime} \times E_{3} \longrightarrow \mathbb{K}$ dada por $A_{1}\left(x_{1}, z_{2}, x_{3}\right) \doteq z_{2}\left(A\left(x_{1}, \cdot, x_{3}\right)\right)$. Assim, segundo a notação anterior, temos:

$$
A_{1}\left(x_{1}, z_{2}, x_{3}\right) \doteq z_{2}\left(A\left(x_{1}, \cdot, x_{3}\right)\right)=\overline{z_{2}}(A)\left(x_{1}, x_{3}\right),
$$


onde $\overline{z_{2}}: \mathscr{L}\left(E_{1}, E_{2}, E_{3}\right) \longrightarrow \mathscr{L}\left(E_{1}, E_{3}\right)$ é dada por $\overline{z_{2}}(M)\left(x_{1}, x_{3}\right) \doteq z_{2}\left(M\left(x_{1}, \cdot, x_{3}\right)\right)$.

Agora defina $A_{2}: E_{1}^{\prime \prime} \times E_{2}^{\prime \prime} \times E_{3} \longrightarrow \mathbb{K}$ por $A_{2}\left(z_{1}, z_{2}, x_{3}\right) \doteq z_{1}\left(A_{1}\left(\bullet, z_{2}, x_{3}\right)\right)$. Isto é:

$$
A_{2}\left(z_{1}, z_{2}, x_{3}\right) \doteq z_{1}\left(A_{1}\left(\bullet, z_{2}, x_{3}\right)\right)=z_{1}\left(\overline{z_{2}}(A)\left(\bullet, x_{3}\right)\right)=\overline{z_{1}}\left[\overline{z_{2}}(A)\right]\left(x_{3}\right)
$$

onde $\overline{z_{1}}: \mathscr{L}\left(E_{1}, E_{3}\right) \longrightarrow \mathscr{L}\left(E_{3}\right)$ é dada por $\overline{z_{1}}(M)\left(x_{3}\right) \doteq z_{1}\left(M\left(\cdot, x_{3}\right)\right)$.

Considere $A_{3}: E_{1}^{\prime \prime} \times E_{2}^{\prime \prime} \times E_{3}^{\prime \prime} \longrightarrow \mathbb{K}$ dada por $A_{3}\left(z_{1}, z_{2}, z_{3}\right) \doteq z_{3}\left(A_{2}\left(z_{1}, z_{2}, \cdot\right)\right)$. Assim:

$$
A_{3}\left(z_{1}, z_{2}, z_{3}\right) \doteq z_{3}\left(A_{2}\left(z_{1}, z_{2}, \cdot\right)\right)=z_{3}\left(\overline{z_{1}}\left(\overline{z_{2}}(A)\right)\right)=\overline{z_{3}} \circ \overline{z_{1}} \circ \overline{z_{2}}(A)
$$

onde, como na construção anterior, definimos $\overline{z_{3}}=z_{3}$.

Segue que aplicação $A_{3}$ como definimos acima também é uma extensão de A para $E_{1}^{\prime \prime} \times E_{2}^{\prime \prime} \times E_{3}^{\prime \prime}$ e satisfaz $\left\|A_{3}\right\|=\|A\|$. Com isso, concluímos que existem 3 ! maneiras de se estender uma aplicação $A \in \mathscr{L}\left(E_{1}, E_{2}, E_{3}\right)$.

Agora apresentaremos o caso geral da construção. Sejam $E_{1}, \ldots, E_{m}$ espaços de Banach sobre um mesmo corpo $\mathbb{K}$ e $A \in \mathscr{L}\left(E_{1}, \ldots, E_{m}\right)$. Para cada $z_{j} \in E_{j}^{\prime \prime}, 1 \leq j \leq m$, definimos a aplicação $\overline{z_{j}}: \mathscr{L}\left(E_{1}, \ldots, E_{j}\right) \longrightarrow \mathscr{L}\left(E_{1}, \ldots, E_{j-1}\right)$ por:

$$
\overline{z_{j}}(M)\left(x_{1}, \ldots, x_{j-1}\right) \doteq z_{j}\left(A\left(x_{1}, \ldots, x_{j-1}, \cdot\right)\right) \text { para cada } M \in \mathscr{L}\left(E_{1}, \ldots, E_{j}\right)
$$

Desta maneira, cada $\overline{z_{j}}$ é linear e contínua, satisfazendo $\left\|\overline{z_{j}}\right\| \leq\left\|z_{j}\right\|$. Definimos a aplicação $\bar{A}: E_{1}^{\prime \prime} \times \cdots \times E_{m}^{\prime \prime} \longrightarrow \mathbb{K}$ por:

$$
\bar{A}\left(z_{1}, \ldots, z_{m}\right) \doteq \overline{z_{1}} \circ \cdots \circ \overline{z_{m}}(A)
$$

Assim, $\bar{A} \in \mathscr{L}\left(E_{1}^{\prime \prime}, \ldots, E_{m}^{\prime \prime}\right)$. Além disso, $\|\bar{A}\| \leq\|A\|$ e $\bar{A}$ é uma extensão de $A$. Isto é, o seguinte diagrama é comutativo:

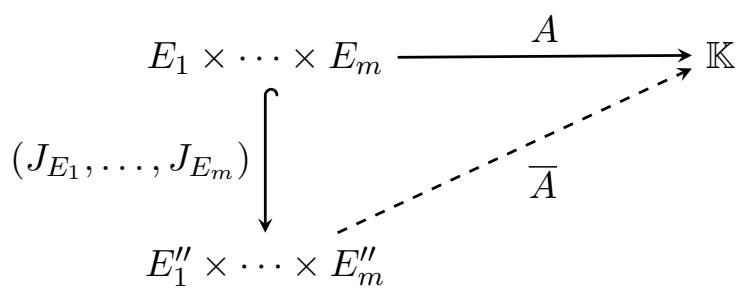

Novamente, observe que a construção de $\bar{A}$ depende da ordem adotada para estender suas variáveis e, portanto, existem $m$ ! maneiras de estender a aplicação $A$. Mais especificamente: 
Definição 4.3.2. Dada $A \in \mathscr{L}\left(E_{1}, \ldots, E_{m}\right)$, para cada $\sigma \in S_{m}$, definimos uma extensão de A por:

$$
\bar{A}^{\sigma}\left(z_{1}, \ldots, z_{m}\right) \doteq \overline{z_{\sigma(1)}} \circ \cdots \circ \overline{z_{\sigma(m)}}(A)
$$

onde cada $\overline{z_{\sigma(j)}}$ é definido de modo à composição acima fazer sentido.

Essas são chamadas de Extensões de Aron-Berner de A, apresentadas em [ABr8].

No próximo capítulo veremos que, mesmo no caso em que $A: E^{m} \longrightarrow \mathbb{K}$ é simétrica, a extensão $\bar{A}$ não precisa ser simétrica. No entanto, essa extensão satisfaz duas importantes propriedades, como diz o próximo resultado:

Proposição 4.3.3. Seja $A \in \mathscr{L}\left({ }^{m} E\right)$. Então:

(a) Os elementos de $E$ comutam com os elementos de $E^{\prime \prime}$ nas entradas de $\bar{A}$

(b) $\bar{A}$ é $w^{*}$-continua na primeira variável.

Demonstração. (a): Sejam $x \in E$ e $z \in E^{\prime \prime}$. Basta mostrar que vale a seguinte igualdade:

$$
\bar{A}\left(z_{1}, \ldots, z_{k-2}, z, x, z_{k+1}, \ldots, z_{m}\right)=\bar{A}\left(z_{1}, \ldots, z_{k-2}, x, z, z_{k+1}, \ldots, z_{m}\right),
$$

onde $z_{1}, \ldots, z_{k-2}, z_{k+1}, \ldots, z_{m} \in E^{\prime \prime}$.

Seja $M=\overline{z_{k+1}} \circ \cdots \circ \bar{z}(A) \in \mathscr{L}\left({ }^{k} E\right)$. Basta mostrar que $\bar{x} \circ \bar{z}(M)=\bar{z} \circ \bar{x}(M)$ em $\mathscr{L}\left({ }^{k-2} E\right)$.

De fato, dados $x_{1}, \ldots, x_{k-2} \in E$ :

$$
\begin{aligned}
\bar{z} \circ \bar{x}(M)\left(x_{1}, \ldots, x_{m-2}\right) & \doteq z\left(\bar{x}(M)\left(x_{1}, \ldots, x_{m-2}, \cdot\right)\right) \\
& =z\left(M\left(x_{1}, \ldots, x_{m-2}, x\right)\right) \\
& \doteq \bar{z}(M)\left(x_{1}, \ldots, x_{m-2}, x\right) \\
& =\left[\bar{z}(M)\left(x_{1}, \ldots, x_{m-2}, \cdot\right)\right](x) \\
& =x\left(\bar{z}(M)\left(x_{1}, \ldots, x_{m-2}, \cdot\right)\right) \\
& =\bar{x} \circ \bar{z}(M)\left(x_{1}, \ldots, x_{m-2}\right) .
\end{aligned}
$$

Logo, os elementos de $E$ comutam com os elementos de $E^{\prime \prime}$ quando são calculados nas entradas de $\bar{A}$.

(b): Dados $z_{1}, \ldots, z_{m} \in E^{\prime \prime}$ e uma rede $\left(x_{\alpha}\right) \subset E$ tal que $x_{\alpha} \stackrel{w^{*}}{\longrightarrow} z_{1}$, temos:

$$
\begin{aligned}
\lim _{\alpha} \bar{A}\left(x_{\alpha}, z_{2}, \ldots, z_{m}\right) & =\lim _{\alpha} \overline{x_{\alpha}} \circ \overline{z_{2}} \circ \cdots \circ \overline{z_{m}}(A) \\
& =\lim _{\alpha} x_{\alpha}\left(\overline{z_{2}} \circ \cdots \circ \overline{z_{m}}(A)\right) \\
& =z_{1}\left(\overline{z_{2}} \circ \cdots \circ \overline{z_{m}}(A)\right)
\end{aligned}
$$




$$
\begin{aligned}
& =\overline{z_{1}} \circ \overline{z_{2}} \circ \cdots \circ \overline{z_{m}}(A) \\
& =\bar{A}\left(z_{1}, z_{2}, \ldots, z_{m}\right) .
\end{aligned}
$$

Observação 4.3.4. Como consequência da demonstração da proposição anterior segue que, para cada $x \in E$ e cada $z \in E^{\prime \prime}$, o seguinte diagrama é comutativo:

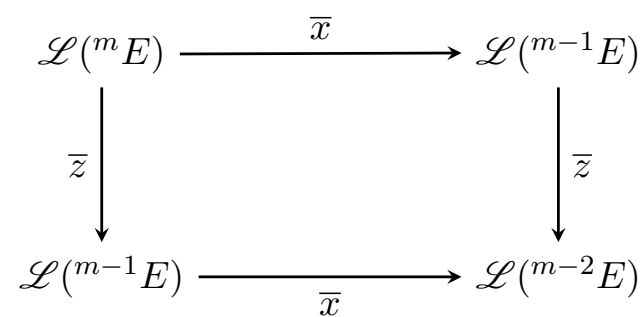

\subsection{Construção de Davie-Gamelin}

A construção que faremos a seguir é baseada no método apresentado por Alexander M. Davie e Theodore W. Gamelin em [DG89].

Sejam $E_{1}, \ldots, E_{m}$ espaços de Banach sobre um mesmo corpo $\mathbb{K}$ e $A \in \mathscr{L}\left(E_{1}, \ldots, E_{m}\right)$. Para cada $z_{j} \in E_{j}^{\prime \prime}$, pelo Teorema de Goldstine existe uma rede $\left(x_{\alpha_{j}}\right) \subset E_{j}$ tal que $x_{\alpha_{j}} \stackrel{w^{*}}{\longrightarrow} z_{j}$.

Observe que, fixado $1 \leq j \leq m$, se $M \in \mathscr{L}\left(E_{1}, \ldots, E_{j}\right)$ e $x_{i} \in E_{i}$, onde $1 \leq i \leq j$, segue que:

$$
\begin{aligned}
\overline{z_{j}}(M)\left(x_{1}, \ldots, x_{j-1}\right) & \doteq z_{j}(\underbrace{M\left(x_{1}, \ldots, x_{j-1}, \cdot\right)}_{\in E_{j}^{\prime}}) \\
& =\lim _{\alpha_{j}} x_{\alpha_{j}}\left(M\left(x_{1}, \ldots, x_{j-1}, \cdot\right)\right) \\
& =\lim _{\alpha_{j}} M\left(x_{1}, \ldots, x_{j-1}, \cdot\right)\left(x_{\alpha_{j}}\right) \\
& =\lim _{\alpha_{j}} M\left(x_{1}, \ldots, x_{j-1}, x_{\alpha_{j}}\right) .
\end{aligned}
$$

Logo, podemos escrever as extensões de Aron-Berner de $A$ da seguinte maneira:

$$
\bar{A}\left(z_{1}, \ldots, z_{m}\right) \doteq \overline{z_{1}} \circ \ldots \circ \overline{z_{m}}(A)=\lim _{\alpha_{1}} \ldots \lim _{\alpha_{m}} A\left(x_{\alpha_{1}}, \ldots, x_{\alpha_{m}}\right)
$$

onde $x_{\alpha_{j}} \stackrel{w^{*}}{\longrightarrow} z_{j}$, para cada $j=1, \ldots, m$.

Esta forma alternativa de escrever a extensão de $A$ é chamada de extensão de Davie-Gamelin. Note que, do mesmo modo, podemos estender $A$ de $m$ ! maneiras distintas, de acordo com a ordem dos limites acima. De forma mais geral: 
Definição 4.4.1. Dadas $A \in \mathscr{L}\left(E_{1}, \ldots, E_{m}\right)$ e $\sigma \in S_{m}$, considere $A_{\sigma} \in \mathscr{L}\left(E_{1}^{\prime \prime}, \ldots, E_{m}^{\prime \prime}\right)$ dada por:

$$
\bar{A}^{\sigma}\left(z_{1}, \ldots, z_{m}\right) \doteq \lim _{\alpha_{\sigma(1)}} \ldots \lim _{\alpha_{\sigma(m)}} A\left(x_{\alpha_{1}}, \ldots, x_{\alpha_{m}}\right)
$$

onde $x_{\alpha_{j}} \stackrel{w^{*}}{\longrightarrow} z_{j}$ para cada $1 \leq j \leq m$. As aplicaçôes $A_{\sigma}$ são chamadas de Extensões de DavieGamelin de A.

Observação 4.4.2. Pelas contas feitas acima, segue que as extensões de Aron-Berner e de DavieGamelin são as mesmas. Para o caso em que $m=2$, segue do Teorema 4.2.7 que as extensões de Arens, de Davie-Gamelin e de Aron-Berner coincidem e portanto, serão utilizadas ao longo do trabalho de acordo com a sua conveniência.

\subsection{Extensões de Polinômios Homogêneos}

Assim como fizemos na Seção 4.2, vamos definir a extensão de um polinômio $m$-homogêneo a partir de sua aplicação $m$-linear simétrica associada. Dada $A \in \mathscr{L}^{s}\left({ }^{m} E\right)$, observe que as extensões $\bar{A}^{\sigma}$ coincidem sobre a diagonal de $\left(E^{\prime \prime}\right)^{m}$. De fato, dado $z \in E^{\prime \prime}$, para cada $\sigma \in S_{m}$ :

$$
\bar{A}^{\sigma} z^{m}=\bar{A}_{\sigma}(z, \ldots, z) \doteq \bar{z} \circ \cdots \circ \bar{z}(A)=\bar{A}^{i d}(z, \ldots, z)
$$

Nessas condições a escolha da ordem de extensão das variáveis é irrelevante e podemos definir a extensão de um polinômio $m$-homogêneo da seguinte maneira:

Definição 4.5.1. Sejam $P \in \mathscr{P}\left({ }^{m} E\right)$ e $A \in \mathscr{L}^{s}\left({ }^{m} E\right)$ sua multilinear simétrica associada. Definimos a extensão de $P$ ao bidual como sendo a função $\bar{P} \in \mathscr{P}\left({ }^{m} E^{\prime \prime}\right)$, dada por:

$$
\bar{P}(z) \doteq \bar{A} z^{m}=\bar{z} \circ \cdots \circ \bar{z}(A)
$$

O seguinte exemplo nos mostra que esta extensão, que é de natureza algébrica, não é necessariamente $w^{*}$-contínua.

Exemplo 4.5.2. Seja $P: l_{2} \longrightarrow \mathbb{K}$ definido por $P(x) \doteq \sum_{n=1}^{\infty} x_{n}^{2}$.

Observe que $A: l_{2}^{2} \longrightarrow \mathbb{K}$ dada por $A(x, y) \doteq \sum_{n=1}^{\infty} x_{n} y_{n}$ é a aplicação bilinear, simétrica $e$ contínua tal que $\widehat{A}=P$. Logo P é um polinômio 2-homogêneo sobre $l_{2}$. Seja $\left(e_{n}\right)$ a base de Schauder canônica de $l_{2}$. Como $l_{2}$ é um espaço reflexivo, as topologias fraca e fraca-estrela coincidem, assim $e_{n} \stackrel{w^{*}}{\longrightarrow} 0$. Por outro lado, para cada $n \in \mathbb{N}$ :

$$
\bar{P}\left(e_{n}\right) \doteq \overline{e_{n}} \circ \overline{e_{n}}(A)=A\left(e_{n}, e_{n}\right)=1 \text {. }
$$


Como $\bar{P}$ estende $P$, temos $\|P\| \leq\|\bar{P}\|$. Além disso, $\bar{P}$ é a restrição de $\bar{A}$ à diagonal de $\left(E^{\prime \prime}\right)^{m}$ assim, devido ao Teorema 3.3.8, temos a seguinte estimativa:

$$
\|\bar{P}\| \leq\|\bar{A}\|=\|A\| \leq \frac{m^{m}}{m !}\|P\|
$$

O próximo teorema nos diz que essa extensão, assim como no caso das extensões de aplicações multilineares, preserva a norma. Este resultado foi demonstrado em 1989 por Alexander M. Davie e por Theodore W. Gamelin em [DG89], onde também foi apresentada uma generalização do Teorema de Goldstine.

Teorema 4.5.3 (Davie-Gamelin). Sejam $P$ um polinômio $m$-homogêneo e $\bar{P}$ sua extensão. Então $\|\bar{P}\|=\|P\|$.

Demonstração. Sejam $P \in \mathscr{P}\left({ }^{m} E\right)$ e $A \in \mathscr{L}^{s}\left({ }^{m} E\right)$ tais que $\widehat{A}=P$. Dado $z \in B_{E^{\prime \prime}}$, pelo Teorema de Goldstine existe $\left(x_{\alpha}\right) \subset B_{E^{\prime \prime}}$ tal que $x_{\alpha} \stackrel{w^{*}}{\longrightarrow} z$. Dado $\varepsilon>0$, como $\bar{A}(\bullet, z, \ldots, z)$ é $w^{*}$-contínuo, existe $x_{1}=x_{\alpha_{1}} \in B_{E}$ tal que:

$$
\left|\bar{A} z^{m}-\bar{A} x_{1} z^{m-1}\right|<\frac{\varepsilon}{m}
$$

Pela Proposição 4.3.3, $\bar{A}\left(x_{1}, \cdot, z, \ldots, z\right)=\bar{A}\left(\cdot, x_{1}, z, \ldots, z\right)$ é $w^{*}$-contínuo. Então existe $x_{2}=$ $x_{\alpha_{2}} \in B_{E}$ tal que:

$$
\left|\bar{A} x_{1} x_{2} z^{m-2}-\bar{A} x_{1} z^{m-1}\right|<\frac{\varepsilon}{m} \quad \text { e } \quad\left|\bar{A} z^{m}-\bar{A} x_{2} z^{m-1}\right|<\frac{\varepsilon}{m} .
$$

Afirmamos que existe uma subsequência $\left(x_{n}\right)$ da rede $\left(x_{\alpha}\right)$ tal que:

$$
\left|\bar{A}\left(x_{i_{1}}, x_{i_{2}}, \ldots, x_{i_{k}}, z, \ldots, z\right)-\bar{A}\left(x_{i_{1}}, x_{i_{2}}, \ldots, x_{i_{k-1}}, z, \ldots, z\right)\right|<\frac{\varepsilon}{m}
$$

sempre que $1 \leq i_{1}<i_{2}<\cdots<i_{k}$, com $1 \leq k<m$.

De fato, suponha $x_{1}, \ldots, x_{n} \in B_{E}$ escolhidos satisfazendo (4.2) e considere o seguinte conjunto:

$$
[\{1, \ldots, n\}]^{<m} \doteq\{F \subset\{1, \ldots, n\}: F \text { é não vazio e }|F|<m\}
$$

onde $|F|$ indica a cardinalidade do conjunto $F$.

Para cada $F=\left\{i_{1}, \ldots, i_{k}\right\} \in[\{1, \ldots, n\}]^{<m}$ com $i_{1}<\cdots<i_{k}$ e $1 \leq k<m$, o operador $\bar{A}\left(\cdot, x_{i_{1}}, \ldots, x_{i_{k}}, z \ldots, z\right)$ é $w^{*}$ contínuo. Logo, existe $x_{\alpha_{F}} \in B_{E}$ tal que, para todo $\alpha \geq \alpha_{F}$ :

$$
\left|\bar{A}\left(x_{\alpha}, x_{i_{1}}, \ldots, x_{i_{k}}, z \ldots, z\right)-\bar{A}\left(x_{i_{1}}, \ldots, x_{i_{k}}, z \ldots, z\right)\right|<\frac{\varepsilon}{m}
$$

Como $[\{1, \ldots, n\}]^{<m}$ é finito e o conjunto de índices da rede $\left(x_{\alpha}\right)$ é dirigido, existe índice $\alpha_{n+1}$ tal que $\alpha_{n+1} \geq \alpha_{F}$ para todo $F \in[\{1, \ldots, n\}]^{<m}$. Denotando $x_{n+1}=x_{\alpha_{n+1}} \in B_{E}$, temos: 


$$
\left|\bar{A}\left(x_{n+1}, x_{i_{1}}, \ldots, x_{i_{k}}, z \ldots, z\right)-\bar{A}\left(x_{i_{1}}, \ldots, x_{i_{k}}, z \ldots, z\right)\right|<\frac{\varepsilon}{m}
$$

sempre que $1 \leq i_{1}<\cdots<i_{k}$, com $1 \leq k<m$.

Logo, de (4.2) e (4.3), para cada $\left\{i_{1}, \ldots, i_{k}\right\} \in[\{1, \ldots, n+1\}]^{<m}$ com $i_{1}<\cdots<i_{k}$, vale que:

$$
\left|\bar{A}\left(x_{i_{1}}, x_{i_{2}}, \ldots, x_{i_{k}}, z, \ldots, z\right)-\bar{A}\left(x_{i_{1}}, x_{i_{2}}, \ldots, x_{i_{k-1}}, z, \ldots, z\right)\right|<\frac{\varepsilon}{m} .
$$

Como $\left(x_{n}\right)$ satisfaz $(4.2)$, sempre que $i_{1}<\cdots<i_{m}$, temos:

$$
\begin{aligned}
\left|\bar{A}\left(x_{i_{1}}, \ldots, x_{i_{m}}\right)-\bar{A} z^{m}\right| \leq & \left|\bar{A}\left(x_{i_{1}}, \ldots, x_{i_{m}}\right)-\bar{A}\left(x_{i_{1}}, \ldots, x_{i_{m-1}}, z\right)\right|+ \\
& +\left|\bar{A}\left(x_{i_{1}}, \ldots, x_{i_{m-1}}, z\right)-\bar{A}\left(x_{i_{1}}, \ldots, x_{i_{m-2}}, z, z\right)\right|+ \\
& +\cdots+\left|\bar{A} x_{i_{1}} z^{m-1}-\bar{A} z^{m}\right| \\
< & \varepsilon
\end{aligned}
$$

Para cada $n \in \mathbb{N}$, considere $y_{n} \doteq \frac{1}{n} \sum_{i=1}^{n} x_{i}$. Assim, cada $y_{n} \in B_{E}$ :

$$
\begin{aligned}
\left|\bar{P}(z)-P\left(y_{n}\right)\right| & =\left|\bar{A} z^{m}-A y_{n}^{m}\right| \\
& =\left|\bar{A} z^{m}-\frac{1}{n^{m}} A\left(x_{1}+\cdots+x_{n}\right)^{m}\right| \\
& =\frac{1}{n^{m}}\left|n^{m} \bar{A} z^{m}-A\left(\sum_{i_{1}=1}^{n} x_{i_{1}}, \ldots, \sum_{i_{m}=1}^{n} x_{i_{m}}\right)\right| \\
& =\frac{1}{n^{m}}\left|\sum_{i_{1}, \ldots, i_{m}=1}^{n} \bar{A} z^{m}-A\left(x_{i_{1}}, \ldots, x_{i_{m}}\right)\right| \\
& \leq \frac{1}{n^{m}} \sum_{i_{1}, \ldots, i_{m}=1}^{n}\left|\bar{A} z^{m}-A\left(x_{i_{1}}, \ldots, x_{i_{m}}\right)\right| \\
& =\frac{1}{n^{m}}\left(S_{1}+S_{2}\right),
\end{aligned}
$$

onde $S_{1}$ denota a soma das parcelas que possuem índices distintos e $S_{2}$ denota a soma das demais parcelas.

Há $n(n-1) \ldots(n-m+1)$ parcelas em $S_{1}$ e, pela simetria de $A$, podemos reordenar os termos $x_{i_{1}}, \ldots, x_{i_{m}}$ de modo que $i_{1}<\cdots<i_{m}$ e, por (4.4), cada uma das parcelas é majorada por $\varepsilon$. Portanto:

$$
\frac{1}{n^{m}} S_{1} \leq \frac{1}{n^{m}} n(n-1) \ldots(n-m+1) \varepsilon \leq \varepsilon .
$$

Como $\|\bar{A}\|=\|A\|$, cada uma das parcelas de $S_{2}$ pode ser majorada por $2\|A\|$. Portanto, tomando $n$ suficientemente grande: 


$$
\begin{aligned}
\frac{1}{n^{m}} S_{2} & \leq\left[n^{m}-n(n-1) \ldots(n-m+1)\right] \frac{1}{n^{m}} 2\|A\| \\
& =2\|A\|\left[1-\frac{n(n-1) \ldots(n-m+1)}{n^{m}}\right] \\
& =2\|A\|\left[1-\left(\frac{n-1}{n}\right)\left(\frac{n-2}{n}\right) \cdots\left(\frac{n-m+1}{n}\right)\right] \\
& =2\|A\|\left[1-\left(1-\frac{1}{n}\right)\left(1-\frac{2}{n}\right) \cdots\left(1-\frac{m-1}{n}\right)\right] \\
& <\varepsilon .
\end{aligned}
$$

Mostramos que, para cada $\varepsilon>0$, existe um $y_{n} \in B_{E}$ tal que $\left|P\left(y_{n}\right)-\bar{P}(z)\right|<\varepsilon$. Logo:

$$
|\bar{P}(z)| \leq\left|P\left(y_{n}\right)-\bar{P}(z)\right|+\left|P\left(y_{n}\right)\right|<\varepsilon+\left|P\left(y_{n}\right)\right| \leq\|P\|+\varepsilon .
$$

Assim, $\|\bar{P}\| \leq\|P\|$. Como a outra desigualdade é sempre válida, concluímos que $\|\bar{P}\|=\|P\|$.

Definição 4.5.4. Considere $\mathscr{P}$ o conjunto de todas as extensões a $E^{\prime \prime}$ de polinômios homogêneos continuos $P: E \longrightarrow \mathbb{K}$. Definimos a topologia polinomial-estrela em $E^{\prime \prime}$ como a menor topologia que torna todos os elementos $\bar{P} \in \mathscr{P}$ contínuos, e será denotada por $\sigma\left(E^{\prime \prime}, \mathscr{P}\right) \doteq p^{*}$.

Das propriedades da topologia gerada por uma família de funções segue que:

$$
z_{\alpha} \stackrel{p^{*}}{\longrightarrow} z \Longleftrightarrow \bar{P}\left(z_{\alpha}\right) \longrightarrow \bar{P}(z), \quad \forall \bar{P} \in \mathscr{P} .
$$

Além disso, as vizinhanças básicas da topologia $p^{*}$ são da forma:

$$
V\left(z ; \overline{P_{1}}, \ldots, \overline{P_{n}} ; \varepsilon\right) \doteq\left\{x^{\prime \prime} \in E^{\prime \prime}:\left|\overline{P_{i}}\left(x^{\prime \prime}\right)-\overline{P_{i}}(z)\right|<\varepsilon, \quad \forall i=1, \ldots, n\right\}
$$

onde $\varepsilon>0, z \in E^{\prime \prime}$ e, para $1 \leq i \leq n, \overline{P_{i}} \in \mathscr{P}$.

Em $E^{\prime \prime}$ podemos considerar a topologia da norma, a topologia $w$, a topologia $w^{*}$ e a topologia $p^{*}$. É claro que convergência em norma implica convergência na topologia $p^{*}$, além disso, como os funcionais lineares contínuos são polinômios 1-homogêneos, convergência na topologia $p^{*}$ implica convergência na topologia $w^{*}$. Em termos de redes, temos:

$$
\text { convergência em norma } \Longrightarrow \text { convergência } p^{*} \Longrightarrow \text { convergência } w^{*}
$$

Observação 4.5.5. Na demonstração do Teorema de Davie-Gamelin (4.5.3) mostramos que cada vizinhança de z da forma:

$$
V(z ; P ; \varepsilon)=\left\{x^{\prime \prime} \in E^{\prime \prime}:\left|\bar{P}\left(x^{\prime \prime}\right)-\bar{P}(z)\right|<\varepsilon\right\}
$$


contém um ponto $y \in B_{E}$. Portanto existe uma rede $\left(x_{\alpha}\right) \subset B_{E}$ tal que $P\left(x_{\alpha}\right) \longrightarrow \bar{P}(z)$.

Além disso, a demonstraçâo pode ser adaptada para um número finito de polinômios homogêneos. Isto é, para cada $z \in B_{E^{\prime \prime}}$, toda vizinhança $V\left(z ; \overline{P_{1}}, \ldots, \overline{P_{n}} ; \varepsilon\right)$ contém algum $x \in B_{E}$.

Assim, obtemos a seguinte generalização do Teorema de Goldistine:

Teorema 4.5.6. Seja E um espaço de Banach. Então ${\overline{B_{E}}}^{p^{*}}=B_{E^{\prime \prime}}$.

Demonstração. Como $w^{*} \leq p^{*}$, pelo Teorema de Goldstine vale que ${\overline{B_{E}}}^{p^{*}} \subset{\overline{B_{E}}}^{w^{*}}=B_{E^{\prime \prime}}$. Pela Observação 4.5.5 segue que $B_{E^{\prime \prime}} \subset{\overline{B_{E}}}^{p^{*}}$.

Corolário 4.5.7. Para cada $z \in B_{E^{\prime \prime}}$, existe uma rede $\left(x_{\alpha}\right) \subset B_{E}$ tal que $x_{\alpha} \stackrel{p^{*}}{\longrightarrow} z$. 


\section{Capítulo 5}

\section{Espaços Regulares e Simetricamente}

\section{Regulares}

Neste capítulo apresentaremos alguns resultados sobre operadores fracamente compactos entre espaços de Banach para que, posteriormente, possamos apresentar duas importantes classes de espaços de Banach. Tais classes estão relacionadas ao fato de que extensões de aplicações multilineares simétricas nem sempre são simétricas.

\subsection{Operadores Fracamente Compactos}

A seguir apresentaremos os resultados básicos sobre operadores fracamente compactos em espaços de Banach. Esta seção é baseada na referência [Meg12].

Definição 5.1.1. Sejam E, F espaços de Banach sobre um mesmo corpo $\mathbb{K}$ e $T: E \longrightarrow F$ um operador linear. Dizemos que $T$ é um operador fracamente compacto se, para cada $B \subset$ E limitado, $T(B) \subset F$ é fracamente relativamente compacto, isto é, se:

$$
\overline{T(B)}^{w} \subset F \text { é w- compacto. }
$$

Observação 5.1.2. Lembre que, dado $B \subset E$, temos:

$$
B \text { é limitado em norma } \Longleftrightarrow B \text { é w-limitado } \Longleftrightarrow x^{\prime}(B) \text { é limitado } \forall x^{\prime} \in E^{\prime} \text {. }
$$

Além disso, se B é convexo, segue do Teorema de Mazur que $\bar{B}^{\|\cdot\|}=\bar{B}^{w}$.

Proposição 5.1.3. Todo operador compacto é fracamente compacto.

Demonstração. Sejam $E, F$ espaços de Banach e $T: E \longrightarrow F$ um operador linear compacto. Dado 
$B \subset E$ limitado, existe $M>0$ tal que $B \subset M B_{E}$. Como $T$ é compacto, o conjunto $\overline{T\left(M B_{E}\right)}\|\cdot\|=$ ${\overline{T\left(M B_{E}\right)}}^{w}$ é compacto e, portanto, fracamente compacto. Logo, $\overline{T(B)}^{w} \subset{\overline{T\left(M B_{E}\right)}}^{w}$ é fracamente compacto.

Proposição 5.1.4. Todo operador fracamente compacto é contínuo.

Demonstração. Seja $T: E \longrightarrow F$ um operador w-compacto. Então ${\overline{T\left(B_{E}\right)}}^{w}$ é fracamente compacto em $F$ e, portanto, limitado. Logo $T\left(B_{E}\right) \subset{\overline{T\left(B_{E}\right)}}^{w}$ é limitado e segue que $T$ é contínuo.

Denotaremos por $\mathscr{K}^{w}(E, F) \doteq\{T \in \mathscr{L}(E, F): T$ é fracamente compacto $\}$ e, analogamente, por $\mathscr{K}(E, F)=\{T \in \mathscr{L}(E, F): T$ é compacto $\}$. As duas proposições anteriores mostram que:

$$
\mathscr{K}(E, F) \subset \mathscr{K}^{w}(E, F) \subset \mathscr{L}(E, F) .
$$

Exemplo 5.1.5. O operador Id $: l_{1} \longrightarrow l_{1}$ é contínuo mas não é fracamente compacto. De fato, como $l_{1}$ não é reflexivo, $B_{l_{1}}$ não é fracamente compacta.

Exemplo 5.1.6. O operador $I d: l_{2} \longrightarrow l_{2}$ é fracamente compacto mas não é compacto. De fato, se $B \subset l_{2}$ é limitado, seja $M>0$ tal que $B \subset M B_{l_{2}}$. Então, pelo Teorema de Mazur:

$$
\bar{B}^{w} \subset{\overline{M B_{l_{2}}}}^{w}={\overline{M B_{l_{2}}}}^{\|\cdot\|}=M B_{l_{2}} .
$$

Logo, $J_{l_{2}}\left(\bar{B}^{w}\right)$ é $w^{*}$-fechado e limitado e, portanto, é $w^{*}$ - compacto. Em particular, segue que $\bar{B}^{w}$ é fracamente compacto.

Assim, mostramos que compacidade, continuidade e compacidade fraca são classes distintas de operadores. A partir de agora estenderemos alguns resultados válidos para operadores compactos.

Proposição 5.1.7. Se $E$ ou $F$ é reflexivo, então todo operador $T \in \mathscr{L}(E, F)$ é fracamente compacto.

Demonstração. Se $E$ é reflexivo, então $B_{E}$ é fracamente compacta e, como $T$ é um operador contínuo, é $w$-w-contínuo. Dado $B \subset E$ limitado, existe $M>0$ tal que $B \subset M B_{E}$. Note que $T\left(M B_{E}\right)$ é fracamente compacto e, como $T(B) \subset T\left(M B_{E}\right)$, temos que $\overline{T(B)}^{w}$ é fracamente compacto

Agora suponha que $F$ é reflexivo. Dado $B \subset E$ limitado, como $T$ é contínuo, existe $M>0$ tal que $T(B) \subset M B_{F}$. Logo, $\overline{T(B)}^{w}$ é fracamente compacto.

A seguir apresentaremos uma caracterização para operadores fracamente compactos: 
Proposição 5.1.8. Sejam E, F espaços de Banach e $T \in \mathscr{L}(E, F)$. São equivalentes:

(a) T é fracamente compacto .

(b) $T\left(B_{E}\right)$ é fracamente relativamente compacto .

(c) Toda sequência limitada $\left(x_{n}\right) \subset E$ admite subsequência $\left(x_{n_{k}}\right)$ tal que $\left(T\left(x_{n_{k}}\right)\right) \subset F$ é fracamente convergente.

Demonstração. (a) $\Longrightarrow(\mathrm{b})$ : Imediato.

(b) $\Longrightarrow$ (c): Seja $\left(x_{n}\right) \subset E$ limitada. Existe $M>0$ tal que, para cada $n \in \mathbb{N},\left\|x_{n}\right\| \leq M$.

Então:

$$
\left(T\left(x_{n}\right)\right) \subset\|T\| M B_{F}={\overline{\|T\| M B_{F}}}^{w} .
$$

O conjunto $J_{F}\left({\overline{\|T\| M B_{F}}}^{w}\right)$ é $w^{*}$ - fechado e limitado, logo é $w^{*}$-compacto. Como $J_{F}: F \longrightarrow F^{\prime \prime}$ é $w$-w*-homeomorfismo sobre a imagem, segue que ${\overline{\|T\| M B_{F}}}^{w}$ é fracamente compacto e, pelo Teorema de Eberlein-Smulian, $\|T\| M B_{F}$ é fracamente sequencialmente compacto.

(c) $\Longrightarrow$ (a): Se $B \subset E$ limitado, existe $M>0$ tal que $B \subset M B_{E}$. Basta provar que $\overline{T\left(M B_{E}\right)}\|\cdot\|$ é fracamente compacto.

De fato, seja $\left(y_{n}\right) \subset \overline{T\left(M B_{E}\right)} \|^{\|\cdot\|}$. Para cada $n \in \mathbb{N}$, existe $x_{n} \in M B_{E}$ tal que $\left\|y_{n}-T\left(x_{n}\right)\right\|<\frac{1}{2^{n}}$. Por hipótese, $\left(x_{n}\right)$ admite uma subsequência fracamente convergente $x_{n_{j}} \stackrel{w}{\longrightarrow} z$. Em particular, como $T$ é um operador $w$-w-contínuo, temos que $T\left(x_{n_{j}}\right) \stackrel{w}{\longrightarrow} T(z)$.

Dados $\varphi \in F^{\prime}$ e $\varepsilon>0$, seja $n_{j_{0}} \in \mathbb{N}$ tal que, para todo $n_{j}>n_{j_{0}}$ :

$$
\left\|y_{n_{j}}-T\left(x_{n_{j}}\right)\right\|<\frac{\varepsilon}{2\|\varphi\|} \quad \text { e } \quad\left|\varphi\left(T\left(x_{n_{j}}\right)\right)-\varphi(T(z))\right|<\frac{\varepsilon}{2} .
$$

Desta maneira, se $n_{j}>n_{j_{0}}$, temos:

$$
\begin{aligned}
\left|\varphi\left(y_{n_{j}}\right)-\varphi(T(z))\right| & \leq\left|\varphi\left(y_{n_{j}}\right)-\varphi\left(T\left(x_{n_{j}}\right)\right)\right|+\left|\varphi\left(T\left(x_{n_{j}}\right)\right)-\varphi(T(z))\right| \\
& \leq\|\varphi\|\left\|y_{n_{j}}-T\left(x_{n_{j}}\right)\right\|+\left|\varphi\left(T\left(x_{n_{j}}\right)\right)-\varphi(T(z))\right| \\
& <\varepsilon .
\end{aligned}
$$

Como $\varphi \in F^{\prime}$ é arbitrário, temos que $y_{n_{j}} \stackrel{w}{\longrightarrow} T(z)$ e, pelo Teorema de Eberlein-Smulian, segue que ${\overline{T\left(M B_{E}\right)}}^{w}$ é fracamente compacto.

Proposição 5.1.9. Sejam E, $F$ espaços de Banach e $T \in \mathscr{K}^{w}(E, F)$. Então $T(E)$ é reflexiva se, e somente se, é fechada.

Demonstração. Se $T(E)$ é reflexiva, então é fechada já que $T(E) \cong(T(E))^{\prime \prime}$. 
Suponha que $T(E)$ seja fechada. Então $T(E)$ é um espaço de Banach e, pelo Teorema da Aplicação Aberta, existe $\delta>0$ tal que:

$$
\delta B_{T(E)} \subset T\left(B_{E}\right) \subset{\overline{T\left(B_{E}\right)}}^{w} .
$$

Logo, ${\overline{\delta B_{T(E)}}}^{w} \subset{\overline{\delta B_{F}}}^{w}$ é fracamente compacto e, como $y \longmapsto \frac{1}{\delta} y$ é $w$-isomorfismo, $B_{T(E)}$ é fracamente compacta. Portanto, $T(E)$ é reflexivo.

Teorema 5.1.10 (Gantmacher). Seja $T \in \mathscr{L}(E, F)$. Então $T$ é um operador fracamente compacto se, e somente se, $T^{\prime \prime}\left(E^{\prime \prime}\right) \subset J_{F}(F)$.

Demonstração. Suponha que $T^{\prime \prime}\left(E^{\prime \prime}\right) \subset J_{F}(F)$. Como a aplicação $J_{F}^{-1}: J_{F}(F) \longrightarrow F$ é $w^{*}-w$ contínua e a aplicação $T^{\prime \prime}: E^{\prime \prime} \longrightarrow F^{\prime \prime}$ é $w^{*}$-w*-contínua, a aplicação $J_{F}^{-1} \circ T^{\prime \prime}$ é $w^{*}$-w-contínua.

Pelo Teorema de Alaoglu, $B_{E^{\prime \prime}}$ é $w^{*}$-compacta e, portanto, $J_{F}^{-1} \circ T^{\prime \prime}\left(B_{E^{\prime \prime}}\right)$ é fracamente compacto. Em particular, temos que $\overline{J_{F}^{-1} \circ T^{\prime \prime} \circ J_{E}\left(B_{E}\right)}{ }^{w} \subset J_{F}^{-1} \circ T^{\prime \prime}\left(B_{E^{\prime \prime}}\right)$ é fracamente compacto.

Como vimos na Seção 4.1, $T=J_{F}^{-1} \circ T^{\prime \prime} \circ J_{E}$ e, pela Proposição 5.1.8, $T$ é fracamente compacto.

Reciprocamente, suponha que $T$ seja fracamente compacto. Então:

$$
\begin{array}{rlrl}
T^{\prime \prime}\left(B_{E^{\prime \prime}}\right) & =T^{\prime \prime}\left(\overline{J_{E}\left(B_{E}\right)} w^{*}\right) & & (\text { Teorema de Goldstine }) \\
& \subset \overline{T^{\prime \prime}\left(J_{E}\left(B_{E}\right)\right)} w^{*} & & \left(T^{\prime \prime} \text { é } w^{*}-w^{*} \text {-contínuo }\right) \\
& =\overline{J_{F}\left(T\left(B_{E}\right)\right)} w^{*} & & \left(T^{\prime \prime} \text { é extensão de } T\right) \\
& \subset \overline{J_{F}\left(\overline{T\left(B_{E}\right)}\right)^{w}} w^{*} & \\
& =J_{F}\left(\overline{T\left(B_{E}\right)}{ }^{w}\right) & & \left(J_{F}\left(\overline{T\left(B_{E}\right)}{ }^{w}\right) \text { é } w^{*} \text {-compacto, logo } w^{*} \text {-fechado }\right) \\
& \subset J_{F}(F) . & &
\end{array}
$$

Proposição 5.1.11. $\mathscr{K}^{w}(E, F)$ é um subespaço vetorial fechado de $\mathscr{L}(E, F)$.

Demonstração. Sejam $S, T \in \mathscr{K}^{w}(E, F)$ e $\lambda \in \mathbb{K}$. Dada uma sequência $\left(x_{n}\right) \subset E$ limitada, existe uma subsequência $\left(x_{n_{k}}\right)$ tal que $T\left(x_{n_{k}}\right) \stackrel{w}{\longrightarrow} y_{1}$ e $S\left(x_{n_{k}}\right) \stackrel{w}{\longrightarrow} y_{2}$. Logo, $(\lambda T+S)\left(x_{n_{k}}\right) \stackrel{w}{\longrightarrow} \lambda y_{1}+y_{2}$ e segue que $\lambda T+S \in \mathscr{K}^{w}(E, F)$.

Seja $T \in \overline{\mathscr{K}^{w}(E, F)}$. Então existe $\left(T_{n}\right) \subset \mathscr{K}^{w}(E, F)$ tal que $T_{n} \longrightarrow T$. Em particular, temos que $T_{n}^{\prime \prime} \longrightarrow T^{\prime \prime}$, pois $\left\|T_{n}^{\prime \prime}-T^{\prime \prime}\right\|=\left\|T_{n}-T\right\| \longrightarrow 0$. Além disso, como cada $T_{n}$ é fracamente compacto, pelo Teorema de Gantmacher, $T_{n}^{\prime \prime}(E) \subset J_{F}(F)$. Assim, para cada $x^{\prime \prime} \in E^{\prime \prime}, T^{\prime \prime}\left(x^{\prime \prime}\right)=\lim T_{n}^{\prime \prime}\left(x^{\prime \prime}\right) \in J_{F}(F)$ e, portanto, $T$ é fracamente compacto.

O próximo resultado nos diz que, assim como no caso dos operadores compactos, o espaço $\mathscr{K}^{w}(E, F)$ também se comporta como um ideal bilateral fechado de $\mathscr{L}(E, F)$ em relação à composição, tanto à esquerda quanto à direita, com operadores lineares contínuos com domínios e imagens apropriados. 
Proposição 5.1.12. Sejam $E_{1}, E_{2}, E_{3}$ espaços de Banach sobre um mesmo corpo $\mathbb{K}, S \in \mathscr{L}\left(E_{1}, E_{2}\right)$, $T \in \mathscr{L}\left(E_{2}, E_{3}\right)$. Se $S$ (ou $T$ ) é fracamente compacto, então $T \circ S$ é fracamente compacto.

Demonstração. Suponha que $S$ seja fracamente compacto. Se $\left(x_{n}\right) \subset E_{1}$ é limitada, então existe $\left(S\left(x_{n_{k}}\right)\right)$ fracamente convergente. Como $T$ é contínuo, segue que $\left(T\left(S\left(x_{n_{k}}\right)\right)\right)$ fracamente convergente e, portanto, $T \circ S$ é fracamente compacto.

Agora suponha que $T$ seja fracamente compacto. Se $\left(x_{n}\right) \subset E_{1}$ é limitada, como $S$ é contínuo, $\left(S\left(x_{n}\right)\right) \subset E_{2}$ é limitada. Logo, existe $\left(T\left(S\left(x_{n_{k}}\right)\right)\right)$ fracamente convergente e, portanto, $T \circ S$ é fracamente compacto.

\subsection{Produto de Arens}

Nesta seção apresentaremos resultados básicos sobre Álgebras de Banach. Posteriormente, a partir destes resultados, apresentaremos uma maneira de estender a multiplicação de uma álgebra de Banach $X$ para o seu bidual de modo a preservar esta estrutura.

Definição 5.2.1. Suponha que $X$ seja um conjunto, que $+e \cdot$ são operações binárias de $X \times X$ em $X$ e que é uma operação binária de $\mathbb{K} \times X$ em $X$ tais que, para todos $x, y, z \in X$ e $\alpha \in \mathbb{K}$ :

(a) $(X,+, \cdot)$ é um espaço vetorial.

(b) $x \cdot(y \cdot z)=(x \cdot y) \cdot z$.

(c) $x \cdot(y+z)=(x \cdot y)+(x \cdot z)$.

(d) $(x+y) \cdot z=(x \cdot z)+(y \cdot z)$.

(e) $\alpha \cdot(x \cdot y)=(\alpha \cdot x) \cdot y=x \cdot(\alpha \cdot y)$.

Então dizemos que $(X,+, \cdot, \cdot)$ é uma álgebra. Dizemos que é uma álgebra com unidade se $X \neq\{0\}$ e se satisfaz:

(f) Existe $e \in X$ tal que, para todo $x \in X, e \cdot x=x \cdot e=x$.

Seja $\|\cdot\|$ uma norma no espaço vetorial $(X,+, \cdot)$. Dizemos que $(X,+, \cdot, \cdot,\|\cdot\|)$ é uma álgebra normada se esta norma satisfaz:

(g) Para cada $x, y \in X,\|x \cdot y\| \leq\|x\|\|y\|$.

Além disso, dizemos que esta é uma álgebra de Banach se $(X,+, \cdot,\|\cdot\|)$ for um espaço de Banach.

Observação 5.2.2. Para evitar toda a notação contida na definição anterior, abreviaremos as multiplicações $\alpha \cdot x$ e $x \cdot y$ para $\alpha x$ e xy, respectivamente. Além disso, abreviaremos a escrita formal da estrutura de $X$ e simplesmente diremos que $X$ é uma álgebra (ou uma álgebra normada ou uma álgebra de Banach). 
Exemplo 5.2.3. $\mathbb{K}$ é uma álgebra de Banach com unidade.

Exemplo 5.2.4. Seja $K$ um espaço topológico compacto Hausdorff. Defina em $\mathscr{C}(K)$ o produto:

$$
(f \cdot g)(x) \doteq f(x) g(x) \quad \text { para todo } x \in K \text {. }
$$

Assim, $\left(\mathscr{C}(K),+, \cdot, \cdot,\|\cdot\|_{\infty}\right)$ é uma álgebra de Banach. Além disso, se $K \neq \emptyset, \mathscr{C}(K)$ tem unidade $e \equiv 1$.

Exemplo 5.2.5. Defina em $l_{\infty}$ o produto:

$$
x y \doteq\left(x_{n} y_{n}\right) \quad \text { onde } x=\left(x_{n}\right), y=\left(y_{n}\right)
$$

Assim, $l_{\infty}$ é uma álgebra de Banach com unidade $e=(1,1,1, \ldots)$.

Exemplo 5.2.6. O espaço $c_{0}$ é uma subálgebra fechada de $l_{\infty}$, logo é uma álgebra de Banach. Entretanto, $c_{0}$ não possui unidade.

Exemplo 5.2.7. Seja $X$ um espaço normado. Defina em $\mathscr{L}(X, X)$ o produto $T S \doteq T \circ S$. Desta maneira $\mathscr{L}(X, X)$ é uma álgebra normada. Se $X$ for um espaço de Banach, então $\mathscr{L}(X, X)$ é uma álgebra de Banach. Além disso, se $X \neq\{0\}$, então $\mathscr{L}(X, X)$ tem unidade $e=i d_{X}$.

Definição 5.2.8. Seja $X$ uma álgebra com unidade e. Dizemos que $x \in X$ é invertível se existe $y \in X$ tal que $x y=y x=e$. Neste caso prova-se que $y$ é único e dizemos que y é o inverso multiplicativo de $x$, denotado por $x^{-1}$.

Definição 5.2.9. Sejam $X$ uma álgebra e $x \in X$. Definimos:

$$
x^{n}= \begin{cases}x & \text { se } n=1 \\ x^{n-1} x & \text { se } n \geq 2 .\end{cases}
$$

Se $X$ é uma álgebra com unidade e, definimos $x^{0} \doteq$ e. Além disso, se $x$ for invertível, definimos $x^{-n} \doteq\left(x^{-1}\right)^{n}$.

A próxima proposição traz algumas propriedades básicas sobre álgebras.

Proposição 5.2.10. Seja X uma álgebra. Então:

(a) Para todo $x \in X, 0 x=x 0=0$.

(b) X tem, no máximo, uma unidade.

Suponha que X tenha unidade e. Então:

(c) $0 \neq e$. 
(d) 0 não é invertível.

(e) Cada $x \in X$ tem, no máximo, um inverso.

(f) Se $x, y, z \in X$ são tais que $y x=x z=e$, então $y=z=x^{-1}$.

(g) Se $x, y \in X$ são invertiveis e $\alpha \in \mathbb{K} \backslash\{0\}$, então $(x y)^{-1}=y^{-1} x^{-1},(\alpha x)^{-1}=\alpha^{-1} x^{-1} e$ $\left(x^{-1}\right)^{-1}=x$.

(h) Se $x \in X$ é invertível, então, para cada $n \in \mathbb{N},\left(x^{n}\right)^{-1}=\left(x^{-1}\right)^{n}=x^{-n}$.

Suponha que $X$ seja uma álgebra normada. Então:

(i) Se $x \in X$ e $n \in \mathbb{N}$, então $\left\|x^{n}\right\| \leq\|x\|^{n}$.

Suponha que $X$ seja uma álgebra normada com unidade e. Então:

(j) $\|e\| \geq 1$.

(k) Se $x \in X$ é invertivel, então $\left\|x^{-1}\right\| \geq\|x\|^{-1}$.

Demonstração. Veja [Meg12], Proposition 3.3.10.

Proposição 5.2.11. Em uma álgebra normada $X$ a multiplicação é uma função contínua.

Demonstração. Basta observar que a multiplicação é uma aplicação bilinear e que $\|x y\| \leq\|x\|\|y\|$ para todos $x, y \in X$. Portanto, pelo Teorema 3.1.2, a multiplicação é contínua.

Definição 5.2.12. Uma involução em uma álgebra $X$ é uma aplicação $x \longmapsto x^{*}$ de $X$ em $X$ tal que, para cada $x, y \in X$ e $\alpha \in \mathbb{K}$ :

(a) $(x+y)^{*}=x^{*}+y^{*}$.

(b) $(\alpha x)^{*}=\bar{\alpha} x^{*}$.

(c) $(x y)^{*}=y^{*} x^{*}$.

(d) $\left(x^{*}\right)^{*}=x$.

Uma álgebra de Banach com uma involução é dita uma $C^{*}$-álgebra se, para cada $x \in X$ :

$$
\left\|x^{*} x\right\|=\|x\|^{2} .
$$

Proposição 5.2.13. Seja $X$ uma $C^{*}$-álgebra. Então:

(a) Para cada $x \in X,\|x\|=\left\|x^{*}\right\|$.

(b) Se $X$ tem unidade, então $x \in X$ é invertivel se, e somente se, $x^{*}$ é invertivel. Neste caso vale $\left(x^{*}\right)^{-1}=\left(x^{-1}\right)^{*}$. 
Demonstração. (a): Dado $x \in X$, note que:

$$
\|x\|^{2}=\left\|x^{*} x\right\|=\left\|\left(x^{*}\right)^{*} x^{*}\right\|=\left\|x^{*}\right\|^{2} .
$$

(b): Dado $x \in X$, note que:

$$
x e^{*}=\left(e x^{*}\right)^{*}=x \quad \text { e } \quad e^{*} x=\left(x^{*} e\right)^{*}=x .
$$

Logo, $e^{*}=e$. Assim, se $x$ é invertível, temos:

$$
\begin{aligned}
x \text { é invertível } & \Longleftrightarrow x x^{-1}=x^{-1} x=e \\
& \Longleftrightarrow\left(x^{-1}\right)^{*} x^{*}=x^{*}\left(x^{-1}\right)^{*}=e \\
& \Longleftrightarrow x^{*} \text { é invertível. }
\end{aligned}
$$

Como vimos na Seção 4.2, as extensões de Arens estendem aplicações bilineares contínuas entre espaços de Banach. Como em uma álgebra de Banach $X$ a multiplicação é bilinear e contínua, podemos considerar suas extensões de Arens para fornecer estrutura de álgebra de Banach para $X^{\prime \prime}$, como faremos abaixo.

A partir de agora nos restringiremos apenas a álgebras comutativas. A seguir reescreveremos a primeira extensão de Arens em uma notação mais adequada para o contexto de álgebras e, finalmente, apresentaremos um exemplo onde esta extensão não preserva comutatividade.

Seja $X$ uma álgebra de Banach. Dados $a, b \in X, S, T \in X^{\prime \prime}$ e $\gamma \in X^{\prime}$, definimos $(a \gamma), T_{\gamma} \in X^{\prime}$ por:

$$
(a \gamma)(b) \doteq \gamma(a b) \quad \text { e } \quad T_{\gamma}(a) \doteq T(a \gamma)
$$

Além disso, definimos uma multiplicação em $X^{\prime \prime}$ da seguinte maneira:

$$
(S T)(\gamma) \doteq S\left(T_{\gamma}\right) \text { para todo } \gamma \in X^{\prime}
$$

Observe que, denotando a multiplicação em $X$ por $A(x, y)=x y$, para cada $a, b \in X, \gamma \in X^{\prime}$ e $T, S \in X^{\prime \prime}$, temos:

$$
\begin{gathered}
A^{t}(\gamma, a)(b) \doteq \gamma(A(a, b))=\gamma(a b) \doteq(a \gamma)(b), \\
A^{t t}(T, \gamma)(a) \doteq T\left(A^{t}(\gamma, a)\right)=T(a \gamma) \doteq T_{\gamma}(a), \\
A^{t t t}(S, T)(\gamma) \doteq S\left(A^{t t}(T, \gamma)\right)=S\left(T_{\gamma}\right) \doteq S T(\gamma)
\end{gathered}
$$

Logo, $A^{t}(\gamma, a)=(a \gamma), A^{t t}(T, \gamma)=T_{\gamma}$ e $A^{t t t}(S, T)=S T$. Dessa maneira, segue que esta mul- 
tiplicação em $X^{\prime \prime}$ estende a multiplicação de $X$. Como as extensões de Arens e de Aron-Berner coincidem, segue da Proposição 4.3.3 que:

(a) A aplicação $(S, T) \longmapsto S T$ é $w^{*}$-contínua na primeira variável.

(b) Os elementos de $X$ comutam com os elementos de $X^{\prime \prime}$.

Em geral, a multiplicação que definimos acima não é $w^{*}$-contínua na segunda variável e o produto de Arens não é comutativo. De fato, essas duas propriedades são equivalentes (veja [Zal05]). Para mostrar que o produto de Arens não é comutativo, apresentamos o seguinte exemplo, presente em [Za191] e [Ren66].

Exemplo 5.2.14. Dada $\left(x_{j}\right)_{j \in \mathbb{Z}} \subset \mathbb{K}$, definimos:

$$
\sum_{j \in \mathbb{Z}}\left|x_{j}\right| \doteq \sup \left\{\sum_{k \in F}\left|x_{k}\right|: F \subset \mathbb{Z} \text { é finito }\right\} .
$$

O espaço $l_{1}(\mathbb{Z}) \doteq\left\{\left(x_{j}\right)_{j \in \mathbb{Z}} \subset \mathbb{K}: \sum_{j \in \mathbb{Z}}\left|x_{j}\right|<\infty\right\}$, munido da norma $\left\|\left(x_{j}\right)\right\| \doteq \sum_{j \in \mathbb{Z}}\left|x_{j}\right|$ é um espaço de Banach.

Considere em $l_{1}(\mathbb{Z})$ a multiplicação definida por $a b \doteq\left((a b)_{j}\right)_{j \in \mathbb{Z}}$, onde $(a b)_{j} \doteq \sum_{i \in \mathbb{Z}} a_{i} b_{j-i}$. Mostraremos apenas que esta multiplicação está bem definida, que é comutativa e tem unidade. As demais propriedades que esta multiplicação deve satisfazer são de fácil verificação.

- A multiplicação está bem definida:

De fato, dados $a, b \in l_{1}(\mathbb{Z})$, se $F, G \subset \mathbb{Z}$ são finitos, então:

$$
\sum_{j \in F} \sum_{i \in G}\left|a_{i}\right|\left|b_{j-i}\right| \leq\|a\|\|b\|
$$

Logo, tomando supremo sobre $F, G \subset \mathbb{Z}$ finitos, obtemos ab $\in l_{1}(\mathbb{Z})$. Em adição, mostramos que $\|a b\| \leq\|a\|\|b\|$.

- $l_{1}(\mathbb{Z})$ é comutativa:

De fato, dados $a, b \in l_{1}(\mathbb{Z})$, para cada $j \in \mathbb{Z}$ :

$$
(a b)_{j}=\sum_{i \in \mathbb{Z}} a_{i} b_{j-i}=\sum_{k \in \mathbb{Z}} b_{k} a_{j-k}=(b a)_{j} .
$$

- $l_{1}(\mathbb{Z})$ tem unidade $e=\left(\delta_{j 0}\right)_{j \in \mathbb{Z}}=(\ldots, 0,0, \underbrace{1}_{0-e ́ s i m a}, 0,0, \ldots)$. 
Logo, $X=l_{1}(\mathbb{Z})$ é uma álgebra de Banach comutativa com unidade. Considere o subespaço de $X^{\prime}=l_{\infty}(\mathbb{Z})$ dado por:

$$
V \doteq\left\{\gamma=\left(\gamma_{n}\right) \in X^{\prime}: L^{-}(\gamma) \doteq \lim _{n \rightarrow-\infty} \gamma_{n} \text { e } L^{+}(\gamma) \doteq \lim _{n \rightarrow+\infty} \gamma_{n} \text { existem }\right\} .
$$

Note que $L^{-}$e $L^{+}$são funcionais lineares contínuos definidos em V. Pelo Teorema de HahnBanach, existem $S^{-}, S^{+} \in X^{\prime \prime}$ extensões de $L^{-}$e de $L^{+}$, respectivamente. Vejamos que $S^{-}$e $S^{+}$ não comutam.

Tome $\gamma=(\ldots,-1,-1,0,1,1, \ldots) \in X^{\prime}=l_{\infty}(\mathbb{Z})$. Dado $a=\left(a_{n}\right) \in l_{1}(\mathbb{Z})$, vamos determinar a $\in \in X^{\prime}$. Para cada $n \in \mathbb{Z}$, temos:

$$
(a \gamma)\left(e_{n}\right)=\sum_{k \in \mathbb{Z}}(a \gamma)_{k}\left(e_{n}\right)_{k}=\sum_{k \in \mathbb{Z}}(a \gamma)_{k} \delta_{n, k}=(a \gamma)_{n}
$$

Por outro lado, temos:

$$
\begin{aligned}
(a \gamma)\left(e_{n}\right) & \doteq \gamma\left(a e_{n}\right) \\
& =\sum_{k \in \mathbb{Z}} \gamma_{k}\left(\sum_{i \in \mathbb{Z}} a_{i} \delta_{n, k-i}\right) \\
& =\sum_{k \in \mathbb{Z}} \gamma_{k} a_{k-n} \\
& =\sum_{k \in \mathbb{Z}} a_{k} \gamma_{n+k} \\
& =\sum_{k>-n} a_{k}-\sum_{k<-n} a_{k} .
\end{aligned}
$$

Portanto, para cada $a \in l_{1}(\mathbb{Z}),(a \gamma)_{n}=\sum_{k>-n} a_{k}-\sum_{k<-n} a_{k}$.

Observe que, para todo $a \in l_{1}(\mathbb{Z})$, a $\in V$ pois a série $\sum_{k \in \mathbb{Z}}\left|a_{k}\right|$ é convergente. Além disso:

$$
S_{\gamma}^{-}(a)=S^{-}(a \gamma)=L^{-}(a \gamma)=\lim _{n \rightarrow-\infty}\left(\sum_{k>-n} a_{k}-\sum_{k<-n} a_{k}\right)=-\sum_{k \in \mathbb{Z}} a_{k}
$$

Logo, $S_{\gamma}^{-}=(\ldots,-1,-1,-1, \ldots)$. De maneira análoga, obtemos $S_{\gamma}^{+}=(\ldots, 1,1,1, \ldots)$. Note que:

$$
\begin{aligned}
& \left(S^{+} S^{-}\right)(\gamma)=S^{+}\left(S_{\gamma}^{-}\right)=L^{+}((\ldots,-1,-1, \ldots))=-1 \\
& \left(S^{-} S^{+}\right)(\gamma)=S^{-}\left(S_{\gamma}^{+}\right)=L^{-}((\ldots, 1,1, \ldots))=1
\end{aligned}
$$

Portanto, o produto de Arens em $l_{1}(\mathbb{Z})$ não é comutativo. 
Observação 5.2.15. Dizer que uma álgebra de Banach $X$ é comutativa é o mesmo que dizer que a aplicação bilinear e contínua $B(x, y) \doteq x y$ é simétrica. Desta maneira, o exemplo anterior nos mostra que as extensões de Arens, em geral, não preservam simetria.

Para mais resultados sobre produto de Arens, veja [IL89],[DH79] e [GI88].

\subsection{Regularidade em Espaços de Banach}

A seguir apresentamos resultados sobre regularidade em espaços de Banach, segundo [Zal05].

Seja $E$ um espaço de Banach sobre $\mathbb{K}$. Dado $A \in \mathscr{L}^{s}\left({ }^{2} E\right)$, considere o operador linear contínuo $T: E \longrightarrow E^{\prime}$ dado por:

$$
T(x) \doteq A(x, \cdot) \quad \text { para todo } x \in E .
$$

Para cada $x, y \in E$, vale que $T(x)(y)=T(y)(x)$. Por este motivo, $T$ é dito o operador linear simétrico associado a $A$.

Observe que a imagem do operador $T^{\prime \prime}: E^{\prime \prime} \longrightarrow E^{\prime \prime \prime}$ coincide com a extensão de Aron-Berner de $A$, isto é:

$$
\bar{A}\left(z_{1}, z_{2}\right) \doteq T^{\prime \prime}\left(z_{1}\right)\left(z_{2}\right), \quad \text { para todos } z_{1}, z_{2} \in E^{\prime \prime}
$$

De fato, dados $z_{2} \in E^{\prime \prime}$ e $x \in E$, temos:

$$
T^{\prime}\left(z_{2}\right)(x) \doteq\left(z_{2} \circ T\right)(x)=z_{2}(A(x, \cdot))=\overline{z_{2}}(A)(x) .
$$

E como definimos $\overline{z_{1}}=z_{1}$ na Seção 4.3 , temos:

$$
T^{\prime \prime}\left(z_{1}\right)\left(z_{2}\right)=\left(z_{1} \circ T^{\prime}\right)\left(z_{2}\right)=z_{1} \circ \overline{z_{2}}(A)=\overline{z_{1}} \circ \overline{z_{2}}(A)=\bar{A}\left(z_{1}, z_{2}\right) .
$$

Na Seção 5.2 vimos que, em geral, $\bar{A}$ não é necessariamente simétrica. De fato, temos o seguinte resultado:

Teorema 5.3.1. Seja $A \in \mathscr{L}^{s}\left({ }^{2} E\right)$. São equivalentes:

(a) $\bar{A}$ é simétrica.

(b) $\bar{A}$ é separadamente $w^{*}$-continua nas duas variáveis.

(c) O operador linear simétrico $T: E \longrightarrow E^{\prime}$ associado a $A$ é fracamente compacto.

Demonstração. $(a) \Longrightarrow(b)$ : Pela Proposição 4.3.3, $\bar{A}$ já é $w^{*}$-contínuo na primeira variável. Como $\bar{A}$ é simétrica, segue que é separadamente $w^{*}$-contínua em cada variável. 
(b) $\Longrightarrow(c)$ : Fixado $z_{1} \in E^{\prime \prime}$, vejamos que $T^{\prime \prime}\left(z_{1}\right) \in J_{E^{\prime}}\left(E^{\prime}\right)$.

De fato, dado $z_{2} \in E^{\prime \prime}$, se $\left(y_{\beta}\right) \subset E$ é uma rede tal que $y_{\beta} \stackrel{w^{*}}{\longrightarrow} z_{2}$, então:

$$
T^{\prime \prime}\left(z_{1}\right)\left(z_{2}\right)=\lim _{\beta} T^{\prime \prime}\left(z_{1}\right)\left(y_{\beta}\right)
$$

pois $\bar{A}=T^{\prime \prime}$ é $w^{*}$-contínuo na segunda entrada.

Observe que, se $x, y \in E$, então:

$$
T^{\prime \prime}(x)(y)=\left(x \circ T^{\prime}\right)(y)=x(y \circ T)=x(T(y))=x(A(y, \cdot))=y(A(x, \cdot)) .
$$

Se $\left(x_{\alpha}\right) \subset E$ é uma rede tal que $x_{\alpha} \stackrel{w^{*}}{\longrightarrow} z_{1}$, então:

$$
\begin{aligned}
T^{\prime \prime}\left(z_{1}\right)\left(z_{2}\right) & =\lim _{\alpha} \lim _{\beta} T^{\prime \prime}\left(x_{\alpha}\right)\left(y_{\beta}\right) \\
& =\lim _{\alpha} \lim _{\beta} y_{\beta}\left(A\left(x_{\alpha}, \cdot\right)\right) \\
& =\lim _{\alpha} z_{2}\left(A\left(x_{\alpha}, \cdot\right)\right) \\
& =z_{2}\left(\lim _{\alpha} T\left(x_{\alpha}\right)\right) \\
& =z_{2}(\lim _{\alpha} \underbrace{T^{\prime \prime}\left(x_{\alpha}\right)}_{\in J_{E^{\prime}}\left(E^{\prime}\right)}) \\
& =z_{2}(\underbrace{T^{\prime \prime}\left(z_{1}\right)}_{\in J_{E^{\prime}}\left(E^{\prime}\right)}) .
\end{aligned}
$$

Logo, $T^{\prime \prime}\left(z_{1}\right) \in J_{E^{\prime}}\left(E^{\prime}\right)$ para todo $z_{1} \in E^{\prime \prime}$. Pelo Teorema de Gantmacher, $T$ é fracamente compacto.

$(c) \Longrightarrow(a)$ : Como $T$ é simétrico, vale que $T=T^{\prime} \circ J_{E}$. De fato, dado $x \in E$ :

$$
T^{\prime} \circ J_{E}(x)=T^{\prime}(x)=x \circ T=T(x) .
$$

Além disso, para cada $z \in E^{\prime \prime}$, segue que $T^{\prime}(z)=T^{\prime \prime}(z) \circ J_{E}$. De fato, se $x \in E$ :

$$
\begin{aligned}
T^{\prime}(z)(x) & =(z \circ T)(x) \\
& =\left(z \circ T^{\prime} \circ J_{E}\right)(x) \\
& =\left(T^{\prime \prime}(z) \circ J_{E}\right)(x) .
\end{aligned}
$$

Como $T$ é fracamente compacto, pelo Teorema de Gantmacher, $T^{\prime \prime}\left(E^{\prime \prime}\right) \subset J_{E^{\prime}}\left(E^{\prime}\right)$. Vejamos que $T^{\prime \prime}=J_{E^{\prime}} \circ T^{\prime}$.

De fato, dados $z \in E^{\prime \prime}, x^{\prime \prime} \in E^{\prime \prime}$ e uma rede $\left(x_{\alpha}\right) \subset E$ tal que $x_{\alpha} \stackrel{w^{*}}{\longrightarrow} x^{\prime \prime}$, temos: 


$$
\begin{aligned}
T^{\prime \prime}(z)\left(x^{\prime \prime}\right) & =\lim _{\alpha} T^{\prime \prime}(z)\left(x_{\alpha}\right) \\
& =\lim _{\alpha} z\left(T^{\prime}\left(x_{\alpha}\right)\right) \\
& =\lim _{\alpha} z \circ T\left(x_{\alpha}\right) \\
& =\lim _{\alpha} T^{\prime}(z)\left(x_{\alpha}\right) \\
& =\lim _{\alpha} x_{\alpha}\left(T^{\prime}(z)\right) \\
& =x^{\prime \prime}\left(T^{\prime}(z)\right) \\
& =J_{E^{\prime}}\left(T^{\prime}(z)\right)\left(x^{\prime \prime}\right) .
\end{aligned}
$$

Observe que, dados $z_{1}, z_{2} \in E^{\prime \prime}$, segue que:

$$
T^{\prime \prime}\left(z_{1}\right)\left(z_{2}\right)=\left[\left(J_{E^{\prime}} \circ T^{\prime}\right)\left(z_{1}\right)\right]\left(z_{2}\right)=\left[J_{E^{\prime}}\left(T^{\prime}\left(z_{1}\right)\right)\right]\left(z_{2}\right)=z_{2}\left(T^{\prime}\left(z_{1}\right)\right)=T^{\prime \prime}\left(z_{2}\right)\left(z_{1}\right) .
$$

Definição 5.3.2. Um espaço de Banach $E$ é dito simetricamente regular se alguma das condiçôes do teorema anterior é satisfeita para toda $A \in \mathscr{L}^{s}\left({ }^{2} E\right)$.

Dizemos que E é regular se todos os operadores $T: E \longrightarrow E^{\prime}$ (simétricos ou não) são fracamente compactos.

Exemplo 5.3.3. Vimos no Exemplo 5.2.14 que $l_{1}(\mathbb{Z})$ com multiplicação dada por $(a b)_{j}=\sum_{i \in \mathbb{Z}} a_{i} b_{j-i}$ é uma álgebra de Banach comutativa cujo produto de Arens não é comutativo. Logo, o espaço $l_{1}(\mathbb{Z})$ não é simetricamente regular. E, portanto, não é regular.

Em contra-partida, G. Godefroy e B. Iochum provaram em [GI88] que $C^{*}$-álgebras e os espaços $\mathscr{C}(K)$ são regulares. Em [Leu96], Denny H. Leung apresentou um exemplo de um espaço simetricamente regular que não é regular.

Observação 5.3.4. Seja E um espaço de Banach. Denotamos por

$$
\mathscr{L}^{s}\left(E, E^{\prime}\right) \doteq\left\{T \in \mathscr{L}\left(E, E^{\prime}\right): T(x, y)=T(y, x) \forall x, y \in E\right\}
$$

o espaço normado dos operadores simétricos e contínuos.

Segundo o isomorfismo do Teorema 3.1.8, segue que $\mathscr{L}^{s}\left({ }^{2} E\right) \cong \mathscr{L}^{s}\left(E, E^{\prime}\right)$ e $\mathscr{L}\left({ }^{2} E\right) \cong \mathscr{L}\left(E, E^{\prime}\right)$. Podemos reescrever a Definição 5.3.2 da seguinte maneira:

(a) E é dito simetricamente regular se $\mathscr{L}^{s}\left(E, E^{\prime}\right) \subset \mathscr{K}^{w}\left(E, E^{\prime}\right)$.

(b) $E$ é dito regular se $\mathscr{L}\left(E, E^{\prime}\right)=\mathscr{K}^{w}\left(E, E^{\prime}\right)$. 
Proposição 5.3.5. Sejam E e $F$ espaços de Banach sobre um mesmo corpo $\mathbb{K}$ tais que $F \cong E^{\prime}$. Então E é regular se, e somente se, $\mathscr{L}(E, F)=\mathscr{K}^{w}(E, F)$.

Demonstração. Seja $\Phi: E^{\prime} \longrightarrow F$ isomorfismo topológico. Se $E$ é regular, dado $T \in L(E, F)$, segue que $\Phi^{-1} \circ T \in \mathscr{L}\left(E, E^{\prime}\right)=\mathscr{K}^{w}\left(E, E^{\prime}\right)$. Pela Proposição 5.1.12, segue que $T=\Phi \circ\left(\Phi^{-1} \circ T\right)$ é fracamente compacto.

Reciprocamente, suponha que $\mathscr{L}(E, F)=\mathscr{K}^{w}(E, F)$. Para cada $T \in \mathscr{L}\left(E, E^{\prime}\right)$, temos que $\Phi \circ T \in \mathscr{L}(E, F)$ e, pela Proposição 5.1.12, segue que $T=\Phi^{-1} \circ(\Phi \circ T)$ é fracamente compacto.

Observação 5.3.6. Sejam E, $F$ espaços de Banach. Então a aplicação $\Psi:(E \times F)^{\prime} \longrightarrow E^{\prime} \times F^{\prime}$ dada por:

$$
\Psi(\alpha) \doteq\left(\left.\alpha\right|_{E \times\{0\}},\left.\alpha\right|_{\{0\} \times F}\right)
$$

é um isomorfismo topológico.

É fácil ver que $\Psi$ está bem definida e é linear. Suponha que $\Psi(\alpha)=0$. Então $\left.\alpha\right|_{E \times\{0\}}=$ $\left.\alpha\right|_{\{0\} \times F}=0$ e, para cada $(x, y) \in E \times F$, temos:

$$
\alpha(x, y)=\alpha(x, 0)+\alpha(0, y)=0 .
$$

Logo, $\Psi$ é injetora.

Dados $x^{\prime} \in E^{\prime}$ e $y^{\prime} \in F^{\prime}$, seja $\alpha: E \times F \longrightarrow \mathbb{K}$ dada por $\alpha(x, y)=x^{\prime}(x)+y^{\prime}(y)$. Então $\alpha$ é linear, contínua e $\Psi(\alpha)=\left(x^{\prime}, y^{\prime}\right)$. Portanto, $\Psi$ é sobrejetora.

Além disso, para cada $\alpha \in(E \times F)^{\prime}$ :

$$
\|\Psi(\alpha)\|=\max \left\{\left\|\left.\alpha\right|_{E \times\{0\}}\right\|,\left\|\left.\alpha\right|_{\{0\} \times F}\right\|\right\} \leq\|\alpha\| .
$$

Logo, $\Psi$ é contínua e, pelo Teorema da Aplicação Aberta, é um isomorfismo topológico.

Teorema 5.3.7. $E \times F$ é regular se, e somente se, $E$ e $F$ são regulares, $\mathscr{L}\left(E, F^{\prime}\right)=\mathscr{K}^{w}\left(E, F^{\prime}\right)$ e $\mathscr{L}\left(F, E^{\prime}\right)=\mathscr{K}^{w}\left(F, E^{\prime}\right)$.

Demonstração. Observe que, dado $T: E \times F \longrightarrow E^{\prime} \times F^{\prime}$ linear e contínuo, os operadores

$$
\begin{array}{ll}
\left.T_{1} \doteq \pi_{E^{\prime}} \circ T\right|_{E \times\{0\}} & \left.T_{2} \doteq \pi_{E^{\prime}} \circ T\right|_{\{0\} \times F} \\
\left.T_{3} \doteq \pi_{F^{\prime}} \circ T\right|_{E \times\{0\}} & \left.T_{4} \doteq \pi_{F^{\prime}} \circ T\right|_{\{0\} \times F}
\end{array}
$$

são lineares e contínuos. Além disso, para cada $(x, y) \in E \times F$ :

$T(x, y)=T(x, 0)+T(0, y)=\left(T_{1}(x), T_{3}(x)\right)+\left(T_{2}(y), T_{4}(y)\right)=\left(T_{1}(x)+T_{2}(y), T_{3}(x)+T_{4}(y)\right)$.

Isto é, o operador $T$ admite a seguinte decomposição: 


$$
T(x, y)=\left(\begin{array}{ll}
T_{1} & T_{2} \\
T_{3} & T_{4}
\end{array}\right)\left(\begin{array}{l}
x \\
y
\end{array}\right),
$$

onde $T_{1} \in \mathscr{L}\left(E, E^{\prime}\right), T_{2} \in \mathscr{L}\left(F, E^{\prime}\right), T_{3} \in \mathscr{L}\left(E, F^{\prime}\right), T_{4} \in \mathscr{L}\left(F, F^{\prime}\right)$.

$(\Longleftarrow)$ Dado $T: E \times F \longrightarrow E^{\prime} \times F^{\prime}$, os operadores $T_{1}, T_{2}, T_{3}, T_{4}$ são fracamente compactos pela Proposição 5.1.12. Se $\left(\left(x_{n}, y_{n}\right)\right) \subset E \times F$ é limitada, segue que $\left(x_{n}\right)$ é limitada e, portanto, existe uma subsequência $\left(x_{n_{k}}\right)$ tal que $T_{1}\left(x_{n_{k}}\right)$ e $T_{3}\left(x_{n_{k}}\right)$ convergem fracamente. Como $\left(y_{n_{k}}\right)$ é limitada, existe $\left(y_{n_{k_{l}}}\right)$ tal que $T_{2}\left(y_{n_{k_{l}}}\right)$ e $T_{4}\left(y_{n_{k_{l}}}\right)$ convergem fracamente. Logo, $T\left(x_{n_{k_{l}}}, y_{n_{k_{l}}}\right)$ é fracamente convergente e segue que $T$ é fracamente compacto. Como $E^{\prime} \times F^{\prime} \cong(E \times F)^{\prime}$, pela Proposição 5.3.5, segue que $E \times F$ é regular.

$(\Longrightarrow)$ Dado $T_{1} \in \mathscr{L}\left(E, E^{\prime}\right)$, o operador $T: E \times F \longrightarrow E^{\prime} \times F^{\prime}$ dado por:

$$
T=\left(\begin{array}{cc}
T_{1} & 0 \\
0 & 0
\end{array}\right)
$$

é fracamente compacto. Logo, $T_{1}=\left.\pi_{E^{\prime}} \circ T\right|_{E \times\{0\}}$ é fracamente compacto e segue que $E$ é regular, isto é, $\mathscr{L}\left(E, E^{\prime}\right)=\mathscr{K}^{w}\left(E, E^{\prime}\right)$.

Com argumento análogo obtemos que $\mathscr{L}\left(E, F^{\prime}\right)=\mathscr{K}^{w}\left(E, F^{\prime}\right)$ e $\mathscr{L}\left(F, E^{\prime}\right)=\mathscr{K}^{w}\left(F, E^{\prime}\right)$ e que $F$ é regular.

Corolário 5.3.8. E é regular se, e somente se, $E \times E$ é regular.

Corolário 5.3.9. Se $E \times E^{\prime}$ é regular, então $E$ é reflexivo.

Demonstração. Se $E \times E^{\prime}$ é regular, então $J_{E}: E \longrightarrow E^{\prime \prime}$ é fracamente compacto. Então $J_{E}\left(B_{E}\right)=$ ${\overline{J_{E}\left(B_{E}\right)}}^{w}$ é fracamente compacto e, em particular, é $w^{*}$-compacta pois $w^{*} \subset w$ em $E^{\prime \prime}$.

Assim, $B_{E}$ é $w$-compacta pois $J_{E}: E \longrightarrow E^{\prime \prime}$ é $w$-w*-homeomorfismo e $E$ é reflexivo.

\section{Teorema 5.3.10.}

(a) Se $E \times E$ é simetricamente regular, então E é regular.

(b) $E \times E$ é regular se, e somente se, $E \times E$ é simetricamente regular.

Demonstração. (a): Dado $T \in \mathscr{L}\left(E, E^{\prime}\right)$, defina $T_{s}: E \longrightarrow E^{\prime}$ por:

$$
T_{s}(x)(y) \doteq T(y)(x) \quad \text { para cada } x, y \in E .
$$

Assim, $T_{s}$ é linear e ainda, para cada $x \in E$ :

$$
\left\|T_{s}(x)\right\|=\sup _{y \in B_{E}}\left\|T_{s}(x)(y)\right\| \leq \sup _{y \in B_{E}}\|T\|\|y\|\|x\| \leq\|T\|\|x\| .
$$


Logo, $T_{s} \in \mathscr{L}\left(E, E^{\prime}\right)$. Considere o operador $S: E \times E \longrightarrow E^{\prime} \times E^{\prime}$ dado por:

$$
S=\left(\begin{array}{cc}
0 & T_{s} \\
T & 0
\end{array}\right)
$$

Assim, $S$ é linear e contínuo. Além disso, para cada $x, y, u, v \in E$ :

$$
\begin{aligned}
S(x, y)(u, v) & =\left(T_{s}(y), T(x)\right)(u, v) \\
& =T_{s}(y)(u)+T(x)(v) \\
& =T_{s}(v)(x)+T(u)(y) \\
& =\left(T_{s}(v), T(u)\right)(x, y) \\
& =S(u, v)(x, y) .
\end{aligned}
$$

Logo, $S$ é simétrico. Como $E \times E$ é simetricamente regular, $S$ é fracamente compacto e, em particular, $T=\left.\pi_{E} \circ S\right|_{E \times\{0\}}$ também o é.

(b): Se $E \times E$ é regular, então $\mathscr{L}\left(E \times E,(E \times E)^{\prime}\right)=\mathscr{K}^{w}\left(E \times E,(E \times E)^{\prime}\right)$. Em particular, $\mathscr{L}^{s}\left(E \times E,(E \times E)^{\prime}\right) \subset \mathscr{K}^{w}\left(E \times E,(E \times E)^{\prime}\right)$ e segue que $E \times E$ é simetricamente regular.

Reciprocamente, suponha que $E \times E$ seja simetricamente regular. Pelo item anterior, $E$ é regular. Pelo Corolário 5.3.8, $E \times E$ é regular. 


\section{Capítulo 6}

\section{Funções Holomorfas em Espaços de}

\section{Banach}

Neste capítulo apresentaremos resultados básicos sobre séries de potências entre espaços de Banach, contidos em [Muj86, Cha85, Nac69]. Estes resultados serão utilizados para definir o conceito de holomorfia em espaços de Banach e, posteriormente, generalizaremos o que foi apresentado no Capítulo 4 para construir extensões de funções holomorfas.

\subsection{Séries de Potências}

Definição 6.1.1. Sejam E e F espaços de Banach sobre um mesmo corpo $\mathbb{K}$. Uma série de potências de E em F em torno de a $\in$ E é uma série de aplicações da forma $\sum_{m=0}^{\infty} P_{m}(x-a)$ onde, para cada $m \in \mathbb{N}_{0}, P_{m} \in \mathscr{P}_{a}\left({ }^{m} E, F\right)$.

Dado $r>0$, como $F$ é completo, a série $\sum_{m=0}^{\infty} P_{m}(x-a)$ converge uniformemente em $B[a, r]$ se, para cada $\varepsilon>0$, existe um $n_{\varepsilon} \in \mathbb{N}$ tal que, para todo $x \in B[a, r]$ :

$$
j, k \geq n_{\varepsilon} \Longrightarrow\left\|\sum_{m=0}^{j} P_{m}(x-a)-\sum_{m=0}^{k} P_{m}(x-a)\right\|<\varepsilon .
$$

O raio de convergência (uniforme) da série $\sum_{m=0}^{\infty} P_{m}(x-a)$ é definido por:

$$
R=\sup \left\{r \geq 0: \sum_{m=0}^{\infty} P_{m}(x-a) \text { converge uniformemente em } B[a, r]\right\} .
$$

Segundo o Teorema 3.3.4, podemos escrever $\sum_{m=0}^{\infty} P_{m}(x-a)=\sum_{m=0}^{\infty} A_{m}(x-a)^{m}$ onde cada $A_{m} \in \mathscr{L}_{a}^{s}\left({ }^{m} E, F\right)$ é tal que $\widehat{A}_{m}=P_{m}$.

Além disso, como em (6.1) podemos ter $r=0$, o raio de convergência de uma série de potências sempre existe. A bola $B(a, R)$ será chamada de bola aberta de convergência uniforme. No caso em 
que $R=+\infty$, diremos que a série é inteira, isto é, a série de potências é uniformemente convergente sobre os subconjuntos limitados de $E$.

Lembre que, dada uma sequência $\left(x_{n}\right) \subset \mathbb{R}$, definimos:

$\liminf _{n \rightarrow \infty} x_{n} \doteq \sup \left\{\inf \left\{x_{m}: m>n\right\}: n \geq 0\right\} \quad$ e $\quad \limsup _{n \rightarrow \infty} x_{n} \doteq \inf \left\{\sup \left\{x_{m}: m>n\right\}: n \geq 0\right\}$

Desta maneira, vale que:

- $\limsup _{n \rightarrow \infty}\left(-x_{n}\right)=\liminf _{n \rightarrow \infty} x_{n}$.

- $\inf _{n} x_{n} \leq \liminf _{n \rightarrow \infty} x_{n} \leq \limsup _{n \rightarrow \infty} x_{n} \leq \sup _{n} x_{n}$.

- Dado $\varepsilon>0$, existe $n_{\varepsilon} \in \mathbb{N}$ tal que, para todo $m>n_{\varepsilon}$ :

$$
\liminf _{n \rightarrow \infty} x_{n}-\varepsilon \leq x_{m} \leq \limsup _{n \rightarrow \infty} x_{n}+\varepsilon
$$

- Se $\left(x_{n}\right) \subset \mathbb{R}$ é uma sequência de termos positivos, então:

$$
\liminf _{n \rightarrow \infty} \frac{x_{n+1}}{x_{n}} \leq \liminf _{n \rightarrow \infty} \sqrt[n]{x_{n}} \leq \limsup _{n \rightarrow \infty} \sqrt[n]{x_{n}} \leq \limsup _{n \rightarrow \infty} \frac{x_{n+1}}{x_{n}}
$$

- Se $\left(x_{n}\right)$ e $\left(y_{n}\right)$ são sequências de números positivos, então:

$$
\limsup _{n} x_{n} y_{n} \leq \limsup _{n} x_{n} \limsup _{n} y_{n}
$$

Teorema 6.1.2. (Cauchy-Hadamard) Seja $R$ o raio de convergência da série $\sum_{m=0}^{\infty} P_{m}(x-a)$. Então:

(a) $\frac{1}{R}=\limsup _{m \rightarrow \infty}\left\|P_{m}\right\|^{\frac{1}{m}}$.

(b) A série converge absolutamente e uniformemente em $B[a, r]$ sempre que $0 \leq r<R$.

Demonstração. Vejamos que $\frac{1}{R} \geq \limsup _{m \rightarrow \infty}\left\|P_{m}\right\|^{\frac{1}{m}}$. Para isso, suponha $R>0$ e note que, dado $0 \leq r<R$, a série converge uniformemente em $B[a, r]$. Considere a seguinte função:

$$
f(x) \doteq \sum_{m=0}^{\infty} P_{m}(x-a) \quad \forall x \in B[a, r]
$$

Tomando $\varepsilon=1$, existe $m_{0} \in \mathbb{N}$ tal que, para todo $x \in B[a, r]$ :

$$
m \geq m_{0} \Longrightarrow\left\|\sum_{j=0}^{m} P_{j}(x-a)-f(x)\right\|<1 .
$$


Dados $m>m_{0}$ e $t \in B[0, r]$, temos que $y=t+a \in B[a, r]$ e:

$$
\begin{aligned}
\left\|P_{m}(t)\right\| & =\left\|P_{m}(y-a)\right\| \\
& \leq\left\|\sum_{j=m}^{\infty} P_{j}(y-a)\right\|+\left\|\sum_{j=m+1}^{\infty} P_{j}(y-a)\right\| \\
& <2 .
\end{aligned}
$$

Logo, $\left\|P_{m}\right\| \leq \frac{2}{r^{m}}$ para todo $m>m_{0}$ e, consequentemente:

$$
\limsup _{m \rightarrow \infty}\left\|P_{m}\right\|^{\frac{1}{m}} \leq \frac{1}{r}
$$

Como a desigualdade acima vale para todo $0 \leq r<R$, tomando o limite quando $r \rightarrow R$, obtemos:

$$
\limsup _{m \rightarrow \infty}\left\|P_{m}\right\|^{\frac{1}{m}} \leq \frac{1}{R}
$$

Agora, vejamos que vale a outra desigualdade. Suponha que $L=\limsup _{m \rightarrow \infty}\left\|P_{m}\right\|^{\frac{1}{m}}<\infty$. Dado $0 \leq r<\frac{1}{L}$, tome $s$ tal que $r<s<\frac{1}{L}$. Assim, existe $m_{0} \in \mathbb{N}$ tal que $\left\|P_{m}\right\|^{\frac{1}{m}}<\frac{1}{s}$ sempre que $m \geq m_{0}$. Se $x \in B[a, r]$, temos:

$$
\left\|P_{m}(x-a)\right\| \leq\left\|P_{m}\right\|\|x-a\|^{m}<\left(\frac{r}{s}\right)^{m} \quad \text { para todo } m \geq m_{0} .
$$

Vejamos que a série $\sum_{m=0}^{\infty} P_{m}(x-a)$ converge uniformemente e absolutamente em $B[a, r]$.

Como $\frac{r}{s}<1$, segue do critério da comparação que $\sum_{m=0}^{\infty}\left\|P_{m}(x-a)\right\|$ é convergente. Dado $\varepsilon>0$, seja $m_{0} \in \mathbb{N}$ tal que:

$$
k>j \geq m_{0} \Longrightarrow\left|\sum_{m=0}^{k}\left(\frac{r}{s}\right)^{m}-\sum_{m=0}^{j}\left(\frac{r}{s}\right)^{m}\right|=\sum_{m=j+1}^{k}\left(\frac{r}{s}\right)^{m}<\varepsilon .
$$

Para todo $x \in B[a, r]$ e para cada $k>j \geq m_{0}$, temos:

$$
\begin{aligned}
\left\|\sum_{m=0}^{k} P_{m}(x-a)-\sum_{m=0}^{j} P_{m}(x-a)\right\| & \leq \sum_{m=j+1}^{k}\left\|P_{m}(x-a)\right\| \\
& \leq \sum_{m=j+1}^{k}\left(\frac{r}{s}\right)^{m}<\varepsilon .
\end{aligned}
$$

Portando, a série é absolutamente e uniformemente convergente em $B[a, r]$. Segue que $R \geq r$ e, passando o limite $r \rightarrow \frac{1}{L}$, obtemos: 


$$
\frac{1}{R} \leq \limsup _{m \rightarrow \infty}\left\|P_{m}\right\|^{\frac{1}{m}}
$$

Note que (b) é uma consequência da demonstração do item $(a)$.

Lema 6.1.3. Seja $\left(c_{m}\right) \subset F$. Se existe $r>0$ tal que $\sum_{m=0}^{\infty} c_{m} \lambda^{m}=0$ para todo $\lambda \in \mathbb{K}$ com $|\lambda| \leq r$, então $c_{m}=0$ para todo $m \in \mathbb{N}_{0}$.

Demonstração. Faremos indução em $m$. Tomando $\lambda=0$, segue que $c_{0}=0$. Suponha que $c_{0}=\cdots=$ $c_{m}=0$, vejamos que $c_{m+1}=0$.

Como a série $\sum_{m=0}^{\infty} c_{m} r^{m}$ é convergente, existe $M>0$ tal que $\left\|c_{m}\right\| r^{m} \leq M$ para todo $m \in \mathbb{N}$. Pela hipótese de indução, para cada $0<|\lambda| \leq r$, temos:

$$
c_{m+1}=\frac{-1}{\lambda^{m+1}} \sum_{j=m+2}^{\infty} c_{j} \lambda^{j}=-\sum_{j=m+2}^{\infty} c_{j} \lambda^{j-m-1} .
$$

Assim, se $0<|\lambda| \leq \frac{r}{2}$, segue que:

$$
\begin{aligned}
\left\|c_{m+1}\right\| & \leq \sum_{j=m+2}^{\infty}\left\|c_{j} \lambda^{j-m-1}\right\| \\
& =\sum_{j=m+2}^{\infty}|\lambda|\left\|c_{j}\right\||\lambda|^{j-m-2} \\
& \leq|\lambda| \sum_{j=m+2}^{\infty} \frac{M}{r^{j}}\left(\frac{r}{2}\right)^{j-m-2} \\
& =|\lambda| M r^{-m-2} \sum_{j=m+2}^{\infty} \frac{1}{2^{j-m-2}} \\
& =2|\lambda| M r^{-m-2} .
\end{aligned}
$$

Tomando o limite quando $|\lambda| \rightarrow 0$, segue que $\left\|c_{m+1}\right\|=0$.

Teorema 6.1.4. Seja $\sum_{m=0}^{\infty} P_{m}(x-a)$ uma série de potências de E em F. Se existe $r>0$ tal que $\sum_{m=0}^{\infty} P_{m}(x-a)=0$ para todo $x \in B(a, r)$, então $P_{m}=0$ para todo $m \in \mathbb{N}_{0}$.

Demonstração. Note que $x \in B(a, r)$ se, e somente se, $x-a=\lambda y \operatorname{com} y \in B(0,1)$ e $|\lambda|<r$.

Assim, para cada $y \in B(0,1)$ e cada $|\lambda|<r$ :

$$
0=\sum_{m=0}^{\infty} P_{m}(x-a)=\sum_{m=0}^{\infty} \lambda^{m} P_{m}(y) .
$$

Pelo Lema anterior, obtemos $P_{m}(y)=0$ para todo $m \in \mathbb{N}_{0}$ e para todo $y \in B(0,1)$. Logo, $P_{m}=0$ para todo $m \in \mathbb{N}_{0}$. 
O seguinte resultado é importante para provar que funções dadas por séries de potências são de classe $\mathscr{C}^{\infty}$ e sua demonstração será omitida.

Teorema 6.1.5. Seja $\sum_{m=0}^{\infty} A_{m}(x-a)^{m}$ uma série de potências com raio de convergência $R>0$ e seja:

$$
f(x)=\sum_{m=0}^{\infty} A_{m}(x-a)^{m} \quad \forall x \in B(a, R) .
$$

Se $y \in B(a, R)$, então existe uma série de potências $\sum_{k=0}^{\infty} B_{k}(x-y)^{k}$ tal que:

$$
f(x)=\sum_{m=0}^{\infty} B_{k}(x-y)^{k} \quad \forall x \in B(a, R-\|y-a\|),
$$

cujo raio de convergência $R^{\prime} \geq R-\|y-a\|$. Além disso, cada $B_{k}$ é dado por:

$$
B_{k}=\sum_{m=k}^{\infty}\left(\begin{array}{l}
m \\
k
\end{array}\right) A_{m}(y-a)^{m-k}
$$

Demonstração. Veja [Cha85], Theorem 11.11.

\subsection{Holomorfia em Espaços de Banach}

Apresentaremos nesta seção uma breve introdução à holomorfia em espaços de Banach, seguindo as referências [Muj86], [Cha85] e [Nac69]. Posteriormente trataremos o problema de estender funções holomorfas. A partir de agora, a menos que se diga o contrário, $E$ e $F$ denotam espaços de Banach sobre $\mathbb{C}$ e $U \subset E$ é um aberto não vazio.

Definição 6.2.1. Uma função $f: U \subset E \longrightarrow F$ é dita holomorfa em $U$ se, para cada $a \in U$, existem $B(a, r) \subset U$ e uma série de potências $\sum_{m=0}^{\infty} P_{m}(x-a)$ de E em $F$ tais que:

$$
f(x)=\sum_{m=0}^{\infty} P_{m}(x-a) \text { uniformemente em } B(a, r) .
$$

Observação 6.2.2. Note que a sequência $\left(P_{m}\right)$ é única em cada a $\in$ U. De fato, suponha que $f(x)=\sum_{m=0}^{\infty} P_{m}(x-a)=\sum_{m=0}^{\infty} Q_{m}(x-a)$ em $B(a, r)$. Então $\sum_{m=0}^{\infty}\left[P_{m}-Q_{m}\right](x-a)=0$ em $B(a, r)$ e, pelo Teorema 6.1 .4 , segue que $P_{m}=Q_{m}$ para todo $m \in \mathbb{N}_{0}$.

Desta maneira podemos escrever $P_{m}=P^{m} f(a)$ para todo $m \in \mathbb{N}_{0}$. A série $\sum_{m=0}^{\infty}\left[P^{m} f(a)\right](x-a)$ é chamada de Série de Taylor de $f$ em a. Denotaremos por $A^{m} f(a)$ a aplicaçâo multilinear simétrica tal que $\widehat{A^{m} f(a)}=P^{m} f(a)$.

Denotaremos por $\mathscr{H}(U, F)$ o espaço formado pelas as funções holomorfas $f: U \longrightarrow F$ com operações definidas pontualmente. No caso em que $F=\mathbb{C}$, escreveremos $\mathscr{H}(U, \mathbb{C})=\mathscr{H}(U)$. 
Exemplo 6.2.3. $\mathscr{P}(E, F) \subset \mathscr{H}(E, F)$.

Basta mostrar que $\mathscr{P}\left({ }^{m} E, F\right) \subset \mathscr{H}(E, F)$ para todo $m \in \mathbb{N}_{0}$. Seja $P=\widehat{A} \in \mathscr{P}\left({ }^{m} E, F\right)$. Dados $x, a \in E$, pela Fórmula Binomial 3.2.6, temos:

$$
P(x)=A x^{m}=A(a+(x-a))^{m}=\sum_{k=0}^{m}\left(\begin{array}{c}
m \\
k
\end{array}\right) A a^{m-k}(x-a)^{k},
$$

onde $A a^{m-k} \in \mathscr{L}^{s}\left({ }^{k} E, F\right)$ para todo $0 \leq k \leq m$.

Logo, P é dado por uma série de potências em torno de cada a $\in$ E. Além disso:

$$
P^{k} P(a)= \begin{cases}\left(\begin{array}{l}
m \\
k
\end{array}\right) A a^{m-k} & \text { se } k \leq m \\
0 & \text { se } k>m .\end{cases}
$$

Teorema 6.2.4 (Princípio da Identidade). Sejam $U \subset E$ um aberto conexo e $f \in \mathscr{H}(U, F)$. Se $f$ é identicamente nula em um aberto não vazio $V \subset U$, então $f$ é identicamente nula em $U$.

Demonstração. Veja [Muj86], Proposition 5.7.

Observação 6.2.5. A Definição 6.2.1 é a definição de holomorfia de Weierstrass. De acordo com a definição de Cauchy-Riemann, uma função $f: U \longrightarrow F$ é dita diferenciável em $U$ se, para cada $a \in U$, existe uma (única) $A \in \mathscr{L}(E, F)$ tal que:

$$
\lim _{h \rightarrow 0} \frac{\|f(a+h)-f(a)-A(h)\|}{\|h\|}=0 .
$$

A aplicação linear A é única, denotada por $D f(a)$ e será chamada de a diferencial de primeira ordem da função $f$ no ponto a.

As duas definições são equivalentes, como veremos no final deste capitulo.

Teorema 6.2.6 (Desigualdade de Cauchy). Sejam $f \in \mathscr{H}(U, F)$ e $f(x)=\sum_{m=0}^{\infty} P_{m}(x-a)$ sua série de Taylor em $B[a, r] \subset U$. Então, para cada $m \in \mathbb{N}_{0}$ :

$$
\left\|P_{m}\right\| \leq \frac{1}{r^{m}} \sup _{\|x-a\|=r}\|f(x)\|
$$

Demonstração. Veja [Muj86], Corollary 7.4.

Observação 6.2.7. Se $f \in \mathscr{H}(U, F), f(x)=\sum_{m=0}^{\infty} P_{m}(x-a)$ é a sua série de Taylor em torno de $a \in U$ e para cada $m \in \mathbb{N}_{0}, A_{m} \in \mathscr{L}^{s}\left({ }^{m} E, F\right)$ é tal que $P_{m}=\widehat{A}_{m}$, a partir do Teorema 3.3.8, podemos escrever a desigualdade de Cauchy da seguinte maneira:

$$
\left\|A_{m}\right\| \leq \frac{1}{r^{m}} \frac{m^{m}}{m !} \sup _{\|x-a\|=r}\|f(x)\|
$$


Definição 6.2.8. Sejam $f \in \mathscr{H}(U, F)$ e $a \in E$.

(a) O raio de limitação de $f$ no ponto a é definido por:

$$
R_{b} f(a) \doteq \sup \left\{r>0: B[a, r] \subset U \text { e }\left.f\right|_{B[a, r]} \text { é limitada }\right\}
$$

(b) O raio de convergência (uniforme) da série de Taylor de $f$ em a será denotado por $R_{c} f(a)$.

(c) A fronteira de U será denotada por $\partial U$. A distância de a até $\partial U$ é dada por:

$$
d_{U}(a) \doteq d(a, \partial U)=\inf \{\|x-a\|: x \in \partial U\}
$$

Quando $U=E$ adotaremos, por conveniência, $d_{U}(a)=+\infty$.

O próximo resultado estabelece uma relação entre o raio de convergência uniforme, o raio de limitação e a distância de um ponto até a fronteira:

Teorema 6.2.9. Sejam $f \in \mathscr{H}(U, F)$ e $a \in E$. Então $R_{b} f(a)=\min \left\{R_{c} f(a), d_{U}(a)\right\}$.

Demonstração. Veja [Muj86], Theorem 7.13.

Definição 6.2.10. Dizemos que $f \in \mathscr{H}(E, F)$ é de tipo limitado se, para cada $B \subset E$ limitado, $f(B) \subset F$ é limitado.

Denotaremos por $\mathscr{H}_{b}(E, F) \doteq\{f \in \mathscr{H}(E, F): f$ é de tipo limitado $\}$.

Observação 6.2.11. Dizemos que $M$ é um espaço de Fréchet se for um espaço vetorial localmente convexo (ELC), metrizável e completo. A topologia de $M$ pode ser dada por uma sequência crescente de seminormas $\left(\|\cdot\|_{j}\right)_{j \in \mathbb{N}}$ de modo que os conjuntos:

$$
U_{j, \varepsilon} \doteq\left\{x \in M:\|x\|_{j} \leq \varepsilon\right\} \quad \text { para todo } j \in \mathbb{N} \text { e todo } \varepsilon>0
$$

formem uma base de vizinhanças do zero.

A sequência $(\|\cdot\|)_{j}$ é chamada de um sistema fundamental de seminormas de $M$ e não é unicamente determinada pela topologia de $M$. Dizemos que o par $\left(M,\left(\|\cdot\|_{j}\right)\right)$ é um espaço de Fréchet graduado.

Um operador linear $T:\left(M_{1},\left(\|\cdot\|_{j}\right)\right) \longrightarrow\left(M_{2},\left(\|\cdot\|_{\sigma}\right)\right)$ entre espaços de Fréchet graduados é contínuo se, para cada $j \in \mathbb{N}$, existem $C>0$ e $\sigma \in \mathbb{N}$ (que podem depender de $j$ ) tais que:

$$
\|T(x)\|_{\sigma} \leq C\|x\|_{j} \quad \text { para todo } x \in M_{1}
$$


O espaço $\mathscr{H}_{b}(E, F)$ é um espaço de Fréchet, cuja topologia é dada pela seguinte família de seminormas:

$$
\|f\|_{r} \doteq \sup _{\|x\| \leq r}\|f(x)\|, \quad r>0
$$

Note que, tomando $r=n$ com $n \in \mathbb{N}$, a sequência de seminormas $\left(\|\cdot\|_{n}\right)_{n \in \mathbb{N}}$ é crescente e define a topologia de $\mathscr{H}_{b}(E, F)$. Logo, $\left(\mathscr{H}_{b}(E, F),\left(\|\cdot\|_{n}\right)\right)$ é graduado.

Além disso, note que se $f \in \mathscr{H}_{b}(E, F)$, segue do Teorema 6.2.9 que $R_{c} f(0)=+\infty$. Esta propriedade será fundamental para definir extensões de funções holomorfas de tipo limitado.

Para mais propriedades de espaços localmente convexos e de espaços de Fréchet, veja [MV9'7].

A partir de agora daremos uma breve introdução a diferenciais de ordem superior de uma função. Lembre que, se $f: U \subset E \longrightarrow F$ é uma função diferenciável, então podemos considerar a aplicação $D f: U \longrightarrow \mathscr{L}(E, F)$. Isto no leva à seguinte definição:

Definição 6.2.12. Seja $U$ um aberto de $E$. Uma função $f: U \longrightarrow F$ é dita duas vezes diferenciável se $f$ é diferenciável e se a função $D f: U \longrightarrow \mathscr{L}(E, F)$ também é diferenciável.

Denotaremos a diferencial da aplicação $D f$ em a por $D^{2} f(a) \doteq D(D f)(a) \in \mathscr{L}(E, \mathscr{L}(E, F))$. Dizemos que $D^{2} f(a)$ é a diferencial de segunda ordem de $f$ no ponto a.

Observação 6.2.13. Observe que, a partir do Teorema 3.1.8, $D^{2} f(a)$ também pode ser vista como um elemento de $\mathscr{L}\left({ }^{2} E, F\right)$ da seguinte maneira:

$$
D^{2} f(a)(x)(y)=D^{2} f(x, y) \quad \text { para todo } x, y \in E .
$$

Definição 6.2.14. Procedendo indutivamente, dizemos que $f: U \subset E \longrightarrow F$ é k-vezes diferenciável se a função $f$ é $(k-1)$-vezes diferenciável e se a aplicação $D^{(k-1)} f: U \longrightarrow \mathscr{L}\left({ }^{(k-1)} E, F\right)$ é diferenciável.

A diferencial da aplicação $D^{(k-1)}$ no ponto a $\in U$ é chamada de diferencial de ordem $k$ de $f$ em a e será denotada por $D^{k} f(a)$.

Uma função é $f: U \subset E \longrightarrow F$ é dita infinitamente diferenciável se for k-vezes diferenciável para todo $k \in \mathbb{N}$.

Observação 6.2.15. Assim como antes, segundo o Teorema 3.1.8, $D^{k} f(a)$ pode ser considerado como elemento de $\mathscr{L}\left(E, \mathscr{L}\left({ }^{(k-1)} E, F\right)\right)$ ou $\mathscr{L}\left({ }^{k} E, F\right)$.

Por conveniência, definimos também $D^{0} f=f$.

Desta maneira, vale a generalização do Teorema de Schwarz:

Teorema 6.2.16. Sejam $U \subset E$ um aberto não vazio e $f: U \longrightarrow F$ uma função k-vezes diferenciável. Então a aplicação $D^{k} f(a) \in \mathscr{L}\left({ }^{k} E, F\right)$ é simétrica para todo a $\in U$. 
A seguir enunciaremos o resultado que prova que as definições de diferenciabilidade e holomorfia são equivalentes.

Teorema 6.2.17. Sejam $E$ e $F$ espaços de Banach complexos, $U \subset E$ um aberto não vazio $e$ $f: U \longrightarrow F$ dada por $f(x)=\sum_{k=0}^{\infty} \widehat{A}_{k}(x-a)$ em torno de $a \in U$. São equivalentes:

(a) f é holomorfa.

(b) f é diferenciável.

(c) f é infinitamente diferenciável.

Se estas condições são satisfeitas, então $D^{k} f(a)=k ! A_{k}$ para todos $a \in U$ e $k \in \mathbb{N}$.

Demonstração. Veja [Muj86], Theorem 14.7

Agora apresentaremos uma outra noção de diferenciabilidade. E, posteriormente, relacionaremos esta nova noção com as diferencias de ordem superior de uma função holomorfa.

Definição 6.2.18. Sejam $U$ um aberto de $E$ e $f: U \longrightarrow F$ uma função. Dados $a \in U$ e $h \in E$, se o seguinte limite existe:

$$
\frac{\partial f}{\partial h}(a) \doteq \lim _{\lambda \rightarrow 0} \frac{f(a+\lambda h)-f(a)}{\lambda}
$$

dizemos que $f$ é G-diferenciável em a, na direção $h$.

Se $f$ é G-diferenciável em a em toda direção $h$, dizemos que $f$ é G-diferenciável em a.

Proposição 6.2.19. Se $f: U \subset E \longrightarrow F$ é diferenciável em $a \in U$, então $f$ é G-diferenciável no ponto a. Além disso, para cada $h \in E$ :

$$
D f(a)(h)=\frac{\partial f}{\partial h}(a)
$$

Demonstração. Seja $h \in E$. Se $h=0$, então:

$$
\lim _{\lambda \rightarrow 0} \frac{f(a+\lambda 0)-f(a)}{\lambda}=\lim _{\lambda \rightarrow 0} \frac{f(a)-f(a)}{\lambda}=0=D f(a)(0) .
$$

Suponha que $h \neq 0$. Em torno de $a$, escrevemos $f(a+\lambda h)-f(h)=D f(a)(\lambda h)+r(\lambda h)$, com $\lim _{\lambda h \rightarrow 0} \frac{r(\lambda h)}{\|\lambda h\|}=0$. Segue que:

$$
\frac{f(a+\lambda h)-f(a)}{\lambda}=D f(a)(h)+\frac{r(\lambda h)}{\lambda}=D f(a)(h)+\frac{r(\lambda h)}{\|\lambda h\|} \frac{\|\lambda h\|}{\lambda} .
$$

Como $\frac{r(\lambda h)}{\|\lambda h\|} \stackrel{\lambda \rightarrow 0}{\longrightarrow} 0$ e $\frac{\|\lambda h\|}{\lambda}$ é limitado, temos:

$$
\frac{\partial f}{\partial h}(a)=\lim _{\lambda \rightarrow 0} \frac{f(a+\lambda h)-f(a)}{\lambda}=\operatorname{Df}(a)(h) .
$$


Observação 6.2.20. Note que se $f: U \subset E \longrightarrow F$ é holomorfa, segue do Teorema 6.2.17 que $f$ é infinitamente diferenciável. Assim, dados $a \in U, h \in E$ e $k \in \mathbb{N}$, temos:

$$
\begin{aligned}
D^{k} f(a)(\underbrace{h, \ldots, h}_{k \text { vezes }}) & =D\left(D^{(k-1)} f\right)(a)(h, \ldots, h) \\
& =\frac{\partial f}{\partial h}\left(D^{(k-1)} f(a)\right)(\underbrace{h, \ldots, h}_{k-1 \text { vezes }}) \\
& =\lim _{\lambda \rightarrow 0} \frac{1}{\lambda}\left[D^{(k-1)} f(a+\lambda h)(h, \ldots, h)-D^{(k-1)} f(a)(h, \ldots, h)\right] .
\end{aligned}
$$




\section{Capítulo 7}

\section{Extensão de Funções Holomorfas}

Dados $E$ e $F$ espaços de Banach complexos, apresentaremos um espaço de Banach $G_{E F}$ que, assim como $E^{\prime \prime}$, contém uma cópia isométrica de $E$ no qual podemos estender funções holomorfas $f$ : $E \longrightarrow F$ de forma natural. Para isso, generalizaremos a construção das extensões de Aron-Berner, posteriormente trataremos o problema de estender funções holomorfas. Também apresentaremos, como feito em [Zal90], uma caracterização desta extensão em termos da continuidade $w^{*}$ do operador diferencial de primeira ordem.

\subsection{O Espaço $G_{E F}$ e a Extensão de Aron-Berner}

Definição 7.1.1. Dados $E$ e $F$ espaços de Banach complexos, definimos $G_{E F} \doteq \mathscr{L}(\mathscr{L}(E, F), F)$.

Observe que, como $F$ é completo, o espaço $G_{E F}$ é um espaço de Banach com a norma de operadores. Além disso, no caso em que $F=\mathbb{C}$, como $G_{E F}=\mathscr{L}(\mathscr{L}(E, \mathbb{C}), \mathbb{C})=E^{\prime \prime}$, existe uma cópia isométrica de $E$ contida em $G_{E \mathbb{C}}$ segundo a aplicação canônica $J_{E}: E \longrightarrow E^{\prime \prime}$. A próxima proposição diz que isso ocorre em geral.

Proposição 7.1.2. E é isometricamente isomorfo a um subespaço (fechado) de $G_{E F}$.

Demonstração. Considere $J_{E, G_{E F}}: E \longrightarrow G_{E F}$ dada por:

$$
J_{E, G_{E F}}(x)(T) \doteq T(x) \text { para todo } T \in \mathscr{L}(E, F) .
$$

Dados $x, y \in E$ e $\lambda \in \mathbb{C}$, para cada $T \in \mathscr{L}(E, F)$, temos:

$$
J_{E, G_{E F}}(\lambda x+y)(T)=T(\lambda x+y)=\lambda T(x)+T(y)=\left[\lambda J_{E, G_{E F}}(x)+J_{E, G_{E F}}(y)\right](T) .
$$

Além disso, para cada $x \in E$, temos:

$$
\left\|J_{E, G_{E F}}(x)\right\|=\sup _{T \in B_{\mathscr{L}(E, F)}}\left\|J_{E, G_{E F}}(x)(T)\right\|=\sup _{\|T\| \leq 1}\|T(x)\| \leq\|x\|
$$


Logo, o operador $J_{E, G_{E F}}$ é linear e contínuo. Para ver que $J_{E, G_{E F}}$ é uma isometria, resta verificar que $\|x\| \leq\left\|J_{E, G_{E F}}(x)\right\|$ para todo $x \in E$.

Dado $x_{0} \in E$ não nulo, pelo Teorema de Hahn-Banach existe $\alpha \in S_{E^{\prime}}$ tal que $\alpha\left(x_{0}\right)=\left\|x_{0}\right\|$. Fixado $y_{0} \in S_{F}$, considere a função $T_{x_{0}}: E \longrightarrow F$ definida por $T_{x_{0}}(x) \doteq \alpha(x) y_{0}$.

Dados $u, v \in E$ e $\lambda \in \mathbb{C}$, temos:

$$
T_{x_{0}}(\lambda u+v)=\alpha(\lambda u+v) y_{0}=\lambda \alpha(u) y_{0}+\alpha(v) y_{0}=\lambda T_{x_{0}}(u)+T_{x_{0}}(v) .
$$

Além disso, para cada $x \in E$ :

$$
\left\|T_{x_{0}}(x)\right\|=\left\|\alpha(x) y_{0}\right\| \leq\|\alpha\|\|x\|\left\|y_{0}\right\|=\|x\| .
$$

Logo, $T_{x_{0}} \in \mathscr{L}(E, F)$ com $\left\|T_{x_{0}}\right\| \leq 1$ e temos que:

$$
\left\|x_{0}\right\|=\left\|T_{x_{0}}\left(x_{0}\right)\right\|=\left\|J_{E, G_{E F}}\left(x_{0}\right)\left(T_{x_{0}}\right)\right\| \leq \sup _{\|T\| \leq 1}\left\|J_{E, G_{E F}}\left(x_{0}\right)(T)\right\|=\left\|J_{E, G_{E F}}\left(x_{0}\right)\right\| .
$$

No caso em que $F=\mathbb{C}$, como a inclusão canônica $J_{E}: E \longrightarrow E^{\prime \prime}$ é uma imersão isométrica, estamos acostumados a identificar $E=J_{E}(E)$ e pensar em $E$ como um subespaço de $E^{\prime \prime}$, escrevendo $E \subset E^{\prime \prime}$ e $x \in E^{\prime \prime}$ para abreviar $J_{E}(x) \in E^{\prime \prime}$. Faremos o mesmo quando $F$ for um espaço de Banach complexo arbitrário. Pela proposição anterior, podemos identificar $E=J_{E, G_{E F}}(E)$ e pensar em $E$ como um subespaço de $G_{E F}$, escrevendo $E \subset G_{E F}$ e $x \in G_{E F}$ para abreviar a notação $J_{E, G_{E F}}(x) \in G_{E F}$.

Na Seção 4.3 vimos como estender uma aplicação $A \in \mathscr{L}\left({ }^{m} E\right)$ para uma aplicação $\bar{A} \in \mathscr{L}\left({ }^{m} E^{\prime \prime}\right)$. Agora generalizaremos esta construção da seguinte maneira: dada uma aplicação $A \in \mathscr{L}\left({ }^{m} E, F\right)$, construiremos uma extensão $\bar{A} \in \mathscr{L}\left({ }^{m} G_{E F}, F\right)$.

Proposição 7.1.3. Sejam $A \in \mathscr{L}\left({ }^{m} E, F\right), T \in G_{E F}$ e $x_{1}, \ldots, x_{m} \in E$. Então:

(a) Para cada $1 \leq k \leq m$, a aplicação $A_{x_{1}, \ldots, x_{k}}: E^{m-k} \longrightarrow F$ definida por:

$$
A_{x_{1}, \ldots, x_{k}}\left(x_{k+1}, \ldots, x_{m}\right) \doteq A\left(x_{1}, \ldots, x_{m}\right)
$$

é $(m-k)$-linear e contínua.

(b) Para todo $m \in \mathbb{N}$, a aplicação $\bar{T}: \mathscr{L}\left({ }^{m} E, F\right) \longrightarrow \mathscr{L}\left({ }^{m-1} E, F\right)$ dada por:

$$
\bar{T}(A)\left(x_{1}, \ldots, x_{m-1}\right) \doteq T\left(A_{x_{1}, \ldots, x_{m-1}}\right),
$$

é linear e contínua com $\|\bar{T}\| \leq\|T\|$.

(c) $\Psi: G_{E F} \longrightarrow \mathscr{L}\left(\mathscr{L}\left({ }^{m} E, F\right), \mathscr{L}\left({ }^{m-1} E, F\right)\right)$ dada por $\Psi(T)=\bar{T}$ é linear e $\|\Psi\| \leq 1$. 
Demonstração. (a): É imediato que $A_{x_{1}, \ldots, x_{k}}$ é $(m-k)$-linear. Dados $x_{k+1}, \ldots, x_{m} \in E$, temos:

$$
\left\|A_{x_{1}, \ldots, x_{k}}\left(x_{k+1}, \ldots, x_{m}\right)\right\|=\left\|A\left(x_{1}, \ldots, x_{m}\right)\right\| \leq\|A\|\left\|x_{1}\right\| \ldots\left\|x_{k}\right\|\left\|x_{k+1}\right\| \ldots\left\|x_{m}\right\| .
$$

$\operatorname{Logo}\left\|A_{x_{1}, \ldots, x_{k}}\right\| \leq\|A\|\left\|x_{1}\right\| \ldots\left\|x_{k}\right\|$.

(b): Dados $A, B \in \mathscr{L}\left({ }^{m} E, F\right), \lambda \in \mathbb{C}$ e $x_{1}, \ldots, x_{m-1} \in E$, temos:

$$
\begin{aligned}
\bar{T}(\lambda A+B)\left(x_{1}, \ldots, x_{m-1}\right) & \doteq T\left((\lambda A+B)_{x_{1}, \ldots, x_{m-1}}\right) \\
& =T\left(\lambda A_{x_{1}, \ldots, x_{m-1}}+B_{x_{1}, \ldots, x_{m-1}}\right) \\
& =\lambda T\left(A_{x_{1}, \ldots, x_{m-1}}\right)+T\left(B_{x_{1}, \ldots, x_{m-1}}\right) \\
& =[\lambda \bar{T}(A)+\bar{T}(B)]\left(x_{1}, \ldots, x_{m-1}\right) .
\end{aligned}
$$

Logo, $\bar{T}$ é linear. Além disso:

$$
\begin{aligned}
\|\bar{T}\| & =\sup _{\|A\| \leq 1}\|\bar{T}(A)\| \\
& =\sup _{\|A\| \leq 1} \sup _{1 \leq x_{i} \| \leq 1}\left\|\bar{T}(A)\left(x_{1}, \ldots, x_{m-1}\right)\right\| \\
& \leq \sup _{\|A\| \leq 1} \sup _{\substack{\left\|x_{i}\right\| \leq 1 \\
1 \leq i \leq m-1}}\|T\|\|A\|\left\|x_{1}\right\| \ldots\left\|x_{m-1}\right\| \\
& =\|T\| .
\end{aligned}
$$

(c): Dados $T, S \in G_{E F}, \lambda \in \mathbb{C}, A \in \mathscr{L}\left({ }^{m} E, F\right)$ e $x_{1}, \ldots, x_{m-1} \in E$, temos:

$$
\begin{aligned}
\Psi(\lambda T+S)(A)\left(x_{1}, \ldots, x_{m-1}\right) & \doteq \overline{(\lambda T+S)}(A)\left(x_{1}, \ldots, x_{m-1}\right) \\
& =(\lambda T+S)\left(A_{x_{1}, \ldots, x_{m-1}}\right) \\
& =\lambda T\left(A_{x_{1}, \ldots, x_{m-1}}\right)+S\left(A_{x_{1}, \ldots, x_{m-1}}\right) \\
& =[\lambda \bar{T}(A)+\bar{S}(A)]\left(x_{1}, \ldots, x_{m-1}\right) \\
& =\lambda \Psi(T)+\Psi(S)](A)\left(x_{1}, \ldots, x_{m-1}\right) .
\end{aligned}
$$

Logo, $\Psi$ é linear e, pelo item anterior, segue que $\|\Psi\| \leq 1$.

Agora estamos em condições de definir

Definição 7.1.4. Seja $A \in \mathscr{L}\left({ }^{m} E, F\right)$. Defina $\bar{A}: G_{E F}^{m} \longrightarrow F$ por:

$$
\bar{A}\left(T_{1}, \ldots, T_{m}\right) \doteq \overline{T_{1}} \circ \cdots \circ \overline{T_{m}}(A) \quad \text { para cada } T_{1}, \ldots, T_{m} \in G_{E F},
$$

onde $\overline{T_{i}}: \mathscr{L}\left({ }^{i} E, F\right) \longrightarrow \mathscr{L}\left({ }^{i-1} E, F\right)$, para todo $1 \leq i \leq m$, é dada como na Proposição 7.1.3 (b). 
Teorema 7.1.5. Dada $A \in \mathscr{L}\left({ }^{m} E, F\right)$, a aplicação $\bar{A} \in \mathscr{L}\left({ }^{m} G_{E F}, F\right)$ e é uma extensão de A. Além disso, $\|\bar{A}\|=\|A\|$.

Demonstração. Fixado $1 \leq j \leq m$, dados $\lambda \in \mathbb{C}$ e $T_{1}, \ldots, T_{m}, S_{j} \in G_{E F}$, se denotarmos por $\bar{T}_{j+1} \circ \cdots \circ \bar{T}_{m}(A)=B \in \mathscr{L}\left({ }^{j} E, F\right)$, segue que:

$$
\begin{aligned}
& \bar{A}\left(T_{1}, \ldots, T_{j-1}, \lambda T_{j}+S_{j}, T_{j+1}, \ldots, T_{m}\right) \doteq \bar{T}_{1} \circ \cdots \circ \bar{T}_{j-1} \circ \overline{\lambda T_{j}+S_{j}} \circ \bar{T}_{j+1} \circ \cdots \circ \bar{T}_{m}(A) \\
& =\bar{T}_{1} \circ \cdots \circ\left(\lambda \bar{T}_{j}+\bar{S}_{j}\right)(B) \\
& =\lambda \bar{T}_{1} \circ \cdots \circ \bar{T}_{j}(B)+\bar{T}_{1} \circ \cdots \circ \bar{S}_{j}(B) \\
& =\lambda \bar{A}\left(T_{1}, \ldots, T_{m}\right)+\bar{A}\left(T_{1}, \ldots, T_{j-1}, S_{j}, T_{j+1}, \ldots, T_{m}\right) .
\end{aligned}
$$

Logo $\bar{A}$ é $m$-linear. Além disso:

$$
\|\bar{A}\|=\sup _{\substack{\left\|T_{j}\right\| \leq 1 \\ 1 \leq j \leq m}}\left\|\bar{A}\left(T_{1}, \ldots, T_{m}\right)\right\|=\sup _{\substack{\left\|T_{j}\right\| \leq 1 \\ 1 \leq j \leq m}}\left\|\bar{T}_{1} \circ \cdots \circ \bar{T}_{m}(A)\right\| \leq\|A\| .
$$

Resta verificar que $\bar{A}$ é uma extensão de $A$, ou seja, queremos provar que o seguinte diagrama é comutativo:

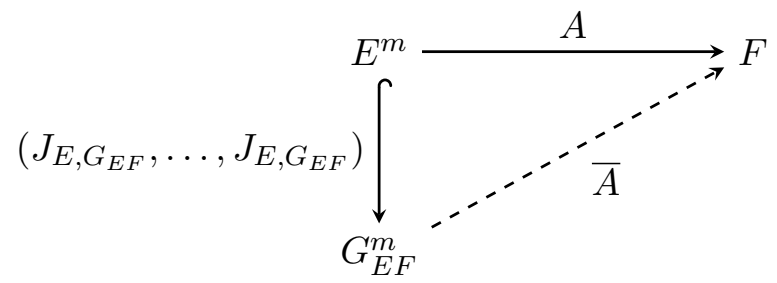

Primeiro, observe que, fixado $1 \leq k \leq m$, se $x \in E \subset G_{E F}, B \in \mathscr{L}\left({ }^{k} E, F\right)$ e $y_{1}, \ldots, y_{k-1} \in E$, temos:

$$
\begin{aligned}
\bar{x}(B)\left(y_{1}, \ldots, y_{k-1}\right) & \doteq x\left(B_{y_{1}, \ldots, y_{k-1}}\right) \\
& =B\left(y_{1}, \ldots, y_{k-1}, x\right) \\
& \doteq B_{x}\left(y_{1}, \ldots, y_{k-1}\right) .
\end{aligned}
$$

Assim, dados $x_{1}, \ldots, x_{m} \in E \subset G_{E F}$, temos:

$$
\begin{aligned}
\bar{A}\left(x_{1}, \ldots, x_{m}\right) & \doteq \bar{x}_{1} \circ \cdots \circ \bar{x}_{m}(A) \\
& =A_{x_{m}, x_{m-1}, \ldots, x_{1}} \\
& =A\left(\cdot, \ldots, \cdot, x_{m}\right)_{x_{m-1}, \ldots, x_{1}} \\
& =A\left(x_{1}, \ldots, x_{m}\right) .
\end{aligned}
$$

Logo, o diagrama é comutativo. Além disso, vale $\|\bar{A}\|=\|A\|$. 
Novamente poderíamos definir $\bar{A}$ de $m$ ! maneiras diferentes apenas permutando a ordem da composição dos operadores $\bar{T}_{j}$. Entretanto, dado um polinômio $P=\widehat{A} \in \mathscr{P}\left({ }^{m} E, F\right)$, a ordem de escolha é irrelevante no seguinte sentido:

$$
\bar{P}(T) \doteq \bar{A}(T, \ldots, T)=\bar{T} \circ \ldots \circ \bar{T}(A)
$$

Outro caso em que a ordem das variáveis é irrelevante é apresentado no próximo resultado.

Proposição 7.1.6. Seja $A \in \mathscr{L}\left({ }^{m} E, F\right)$. Os elementos de $E$ comutam com os elementos de $G_{E F}$ nas entradas de $\bar{A}$.

Demonstração. Sejam $a \in E$ e $T \in G_{E F}$. Basta mostrar que vale a seguinte igualdade:

$$
\bar{A}\left(T_{1}, \ldots, T_{k-2}, T, a, T_{k+1}, \ldots, T_{m}\right)=\bar{A}\left(T_{1}, \ldots, T_{k-2}, a, T, T_{k+1}, \ldots, T_{m}\right),
$$

onde $T_{1}, \ldots, T_{k-2}, T_{k+1}, \ldots, T_{m} \in G_{E F}$.

Seja $M=\overline{T_{k+1}} \circ \cdots \circ \bar{T}(A) \in \mathscr{L}\left({ }^{k} E, F\right)$. Basta mostrar que $\bar{a} \circ \bar{T}(M)=\bar{T} \circ \bar{a}(M)$ em $\mathscr{L}\left({ }^{k-2} E, F\right)$.

De fato, dados $y_{1}, \ldots, y_{m-2} \in E$, temos:

$$
\begin{aligned}
\bar{a} \circ \bar{T}(A)\left(y_{1}, \ldots, y_{k-2}\right) & \doteq a\left(\bar{T}(A)_{y_{1}, \ldots, y_{k-2}}\right) \\
& =a(\underbrace{\bar{T}(A)\left(y_{1}, \ldots, y_{k-2}, \cdot\right)}_{\in \mathscr{L}(E, F)}) \\
& \doteq \bar{T}(A)\left(y_{1}, \ldots, y_{k-2}, a\right) \\
& \doteq T\left(A_{y_{1}, \ldots, y_{k-2}, a}\right) \\
& =T(\underbrace{A\left(y_{1}, \ldots, y_{k-2}, a\right)}_{\in \mathscr{L}(E, F)}) \\
& \doteq T\left(a\left(A_{y_{1}, \ldots, y_{k-2}}\right)\right) \\
& \doteq T\left(\bar{a}(A)\left(y_{1}, \ldots, y_{k-2}\right)\right) \\
& =\bar{T} \circ \bar{a}(A)\left(y_{1}, \ldots, y_{k-2}\right)
\end{aligned}
$$

Observação 7.1.7. Como consequência da demonstração da proposição anterior segue que, para cada $a \in E$ e cada $T \in G_{E F}$, o seguinte diagrama é comutativo:

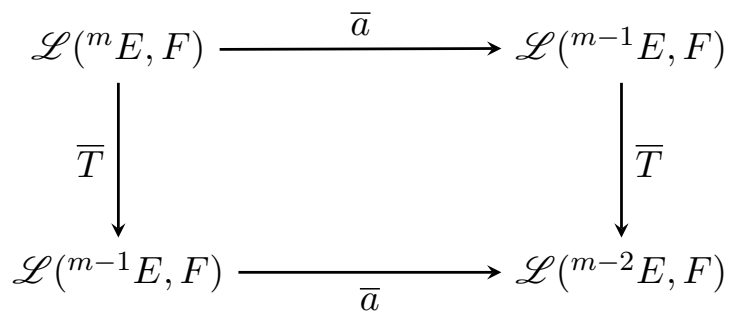


A comutatividade parcial descrita no resultado anterior pode ser vista como uma propriedade reminiscente do produto de Arens. Outra característica que esta extensão compartilha com o produto de Arens é uma forma de continuidade na primeira variável, como descrevemos a seguir.

Considere em $G_{E F}$ a topologia $\sigma\left(G_{E F}, \mathscr{F}\right)$, gerada pela família $\mathscr{F}=\left\{\bar{u} \in \mathscr{L}\left(G_{E F}, F\right): u \in \mathscr{L}(E, F)\right\}$. Para cada $u \in \mathscr{L}(E, F)$ e cada $T \in G_{E F}$, vale que $\bar{u}(T) \doteq \bar{T}(u)=T(u)$.

Desta maneira, cada $\bar{u}$ é contínua em relação à topologia $\sigma\left(G_{E F}, \mathscr{F}\right)$. De fato, dada uma rede $T_{\alpha} \stackrel{\sigma\left(G_{E F}, \mathscr{F}\right)}{\longrightarrow} T$ em $G_{E F}$, temos:

$$
\bar{u}\left(T_{\alpha}\right)=T_{\alpha}(u) \longrightarrow T(u)=\bar{u}(T) .
$$

Consequentemente, se $A \in \mathscr{L}\left({ }^{m} E, F\right)$, então:

$$
\begin{aligned}
\bar{A}\left(T_{1}, \ldots, T_{m}\right) & \doteq \bar{T}_{1} \circ \cdots \circ \bar{T}_{m}(A) \\
& =\bar{T}_{1}(\underbrace{\bar{T}_{2} \circ \cdots \circ \bar{T}_{m}(A)}_{\in \mathscr{L}(E, F)}) \\
& =\overline{\left(\bar{T}_{2} \circ \cdots \circ \bar{T}_{m}\right)(A)}\left(T_{1}\right) .
\end{aligned}
$$

Logo, $\bar{A}$ é $\sigma\left(G_{E F}, \mathscr{F}\right)$ - contínuo na primeira entrada.

\subsection{Extensão de funções holomorfas de tipo limitado}

Como funções de tipo limitado têm raio de convergência uniforme infinito, dada $f \in \mathscr{H}_{b}(E, F)$ podemos considerar sua série de Taylor centrada na origem e escrever $f(x)=\sum_{k=0}^{\infty} \frac{D^{k} f(0)}{k !}(x, \ldots, x)$ para todo $x \in E$. Assim, temos uma candidata natural para estender $f$ para $G_{E F}$, dada por:

$$
\bar{f}(T) \doteq \sum_{k=0}^{\infty} \frac{1}{k !} \overline{D^{k} f(0)}(T, \ldots, T), \quad \text { para todo } T \in G_{E F}
$$

O próximo resultado nos diz que $\bar{f}$ é uma função holomorfa de tipo limitado e que estende $f$ para $G_{E F}$.

Teorema 7.2.1. A função $\Psi: \mathscr{H}_{b}(E, F) \longrightarrow \mathscr{H}_{b}\left(G_{E F}, F\right)$ dada por $\Psi(f) \doteq \bar{f}$ é linear e contínua. Além disso, $\bar{f}$ é extensão de $f$.

Demonstração. Dadas $f, g \in \mathscr{H}_{b}(E, F)$ e $\lambda \in \mathbb{C}$, escrevemos:

$$
f(x)=\sum_{k=0}^{\infty} \frac{1}{k !} D^{k} f(0)(x, \ldots, x) \quad e \quad g(x)=\sum_{k=0}^{\infty} \frac{1}{k !} D^{k} g(0)(x, \ldots, x) .
$$

Então, para cada $T \in G_{E F}$ : 


$$
\begin{aligned}
\overline{\lambda f+g}(T) & \doteq \sum_{k=0}^{\infty} \frac{1}{k !} \overline{D^{k}(\lambda f+g)(0)}(T, \ldots, T) \\
& =\sum_{k=0}^{\infty} \frac{1}{k !}\left[\lambda \overline{D^{k} f(0)}+\overline{D^{k} g(0)}\right](T, \ldots, T) \\
& =(\lambda \bar{f}+\bar{g})(T) .
\end{aligned}
$$

Dado $x \in E \subset G_{E F}$, temos:

$$
\bar{f}(x) \doteq \sum_{k=0}^{\infty} \frac{1}{k !} \overline{D^{k} f(0)}(x, \ldots, x)=\sum_{k=0}^{\infty} \frac{1}{k !} D^{k} f(0)(x, \ldots, x)=f(x)
$$

Logo, o seguinte diagrama é comutativo:

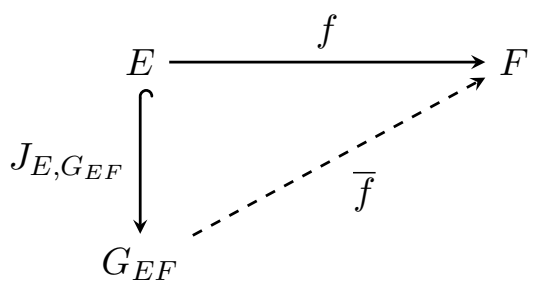

Vejamos agora que $\bar{f}$ é holomorfa de tipo limitado. Dado $T \in G_{E F}$, usando a Desigualdade de Cauchy (6.2.6) para $B[0, \rho]$, temos:

$$
\begin{aligned}
\|\bar{f}(T)\| & \leq \sum_{k=0}^{\infty}\left\|\frac{1}{k !} \overline{D^{k} f(0)}(T, \ldots, T)\right\| \\
& \leq \sum_{k=0}^{\infty}\left\|\frac{1}{k !} \overline{D^{k} f(0)}\right\|\|T\|^{k} \\
& =\sum_{k=0}^{\infty}\left\|\frac{1}{k !} D^{k} f(0)\right\|\|T\|^{k} \\
& \leq \sum_{k=0}^{\infty} \frac{\|T\|^{k}}{k !} \frac{k^{k}}{\rho^{k}} \sup _{\|x\| \leq \rho}\|f(x)\| .
\end{aligned}
$$

A sequência $\left(\frac{k^{k}}{k !}\right)^{1 / k}$ é crescente e $\lim _{k}\left(\frac{k^{k}}{k !}\right)^{1 / k}=e$. Assim, se tomarmos $\rho>e\|T\|$, a série $\sum_{k=0}^{\infty} \frac{k^{k}}{k !} \frac{\|T\|^{k}}{\rho^{k}}$ é convergente e, como $f$ é de tipo limitado:

$$
\|\bar{f}(T)\| \leq C \sup _{\|x\| \leq \rho}\|f(x)\|<\infty
$$

onde $C$ é a soma da série.

Além disso, para cada $S \in G_{E F}$ tal que $\|S\| \leq\|T\|$, tomando $\rho>e\|T\|$ e utilizando o mesmo 
argumento, segue que $\|\bar{f}(S)\| \leq C \sup _{\|x\| \leq \rho}\|f(x)\|$. Logo, para todo $r>0$ e todo $\rho>$ er, vale:

$$
\|\bar{f}\|_{r} \doteq \sup _{\|T\| \leq r}\|\bar{f}(T)\| \leq C \sup _{\|x\| \leq \rho}\|f(x)\| \doteq\|f\|_{\rho}
$$

Portanto, $\bar{f} \in \mathscr{H}_{b}\left(G_{E F}, F\right)$ e a aplicação $\Psi$ é contínua.

Definição 7.2.2. Seja $f \in \mathscr{H}_{b}(E, F)$. Considere a função $\bar{f}: G_{E F} \longrightarrow F$ dada por:

$$
\bar{f}(T) \doteq \sum_{k=0}^{\infty} \frac{1}{k !} \overline{D^{k} f(0)}(T, \ldots, T)
$$

Da demonstração do Teorema 7.2.1 segue que, se $f \in \mathscr{H}(E, F)$ tem raio de convergência uniforme $R_{c} f(0)$ finito, $f$ pode ser estendida para uma bola de raio $R \geq \frac{R_{c} f(0)}{e}$ em torno de $0 \in G_{E F}$. No caso em que $E=\mathbb{C}$, segue que $R=R_{c} f(0)$ e quando $F=\mathbb{C}$, Davie e Gamelin mostraram que isto também ocorre [DG89].

Exemplo 7.2.3. Quando $F=\mathbb{C}$, temos $G_{E F}=E^{\prime \prime}$ e a extensão descrita acima é a mesma a extensão de Aron-Berner, apresentada em [AB78]. Além disso, quando $F=\mathbb{C}$ e E é reflexivo, a extensão é trivial.

Exemplo 7.2.4. Seja $X$ uma álgebra de Banach comutativa e fixe $\gamma \in X^{\prime}$. Então a aplicação $\phi: X \times X \longrightarrow \mathbb{C}$ dada por $\phi(x, y) \doteq \gamma(x y)$ é bilinear, simétrica e continua. Dados $T, S \in X^{\prime \prime}$, as duas possiveis extensões de $\phi$ ao bidual são dadas pelo produto de Arens, como verificamos a seguir.

Conforme a notação apresentada na Seção 5.2 ,para cada $x \in X$, temos:

$$
\bar{T}(\phi)(x) \doteq T(\phi(x, \cdot))=T(x \gamma) \doteq T_{\gamma}(x)
$$

Logo:

$$
\bar{S} \circ \bar{T}(\phi)=\bar{S}\left(T_{\gamma}\right) \doteq(S T)(\gamma)
$$

Analogamente, a outra extensão de $\phi$ é dada por $\bar{T} \circ \bar{S}(\phi)=(T S)(\gamma)$.

Exemplo 7.2.5. Sejam $X$ uma álgebra de Banach comutativa e $\gamma \in X^{\prime}$. Considere a função $f: X^{2} \longrightarrow \mathbb{C}$ dada por $f(x, y) \doteq \gamma(x y)$.

Considere a aplicação $A: X^{2} \times X^{2} \longrightarrow \mathbb{C}$ dada por:

$$
A((a, b),(c, d)) \doteq \frac{1}{2}[\gamma(a d)+\gamma(b c)]
$$

Assim, A é uma aplicação bilinear e simétrica. Além disso, para cada $(a, b),(c, d) \in E$, temos:

$$
\|A((a, b),(c, d))\|=\frac{1}{2}(\|\gamma(a d)+\gamma(b c)\|)
$$




$$
\begin{aligned}
& \leq \frac{1}{2}(\|\gamma\|\|a\|\|d\|+\|\gamma\|\|b\|\|c\|) \\
& \leq \frac{\|\gamma\|}{2}(\max \{\|a\|,\|b\|\}(\|d\|+\|c\|)) \\
& \leq \frac{\|\gamma\|}{2}(\max \{\|a\|,\|b\|\} 2 \max \{\|c\|,\|d\|\}) \\
& =\|\gamma\|\|(a, b)\|\|(c, d)\| .
\end{aligned}
$$

Logo, A é contínua e ainda, como X é comutativa:

$$
A((a, b),(a, b))=\frac{1}{2}[\gamma(a b)+\gamma(b a)]=\gamma(a b) \doteq f(a, b) .
$$

Isto mostra que $f$ é um polinômio 2-homogêneo e, portanto, é holomorfa. Em particular, para cada $\phi \in\left(X^{2}\right)^{\prime \prime}:$

$$
\bar{f}(\phi)=\bar{A}(\phi, \phi) \doteq \bar{\phi} \circ \bar{\phi}(A)
$$

Pela Observação 5.3.6, podemos identificar $\left(X^{2}\right)^{\prime \prime}=X^{\prime \prime} \times X^{\prime \prime}$ e, dado $\left(x^{\prime \prime}, y^{\prime \prime}\right) \in X^{\prime \prime} \times X^{\prime \prime}$, temos $(\alpha, \beta)(x, y)=x^{\prime \prime}(x)+y^{\prime \prime}(y)$. Denotando $\phi=(S, T) \in X^{\prime \prime} \times X^{\prime \prime}$ em (7.1), temos:

$$
\bar{f}(S, T)=\overline{(S, T)} \circ \overline{(S, T)}(A) .
$$

Dados $(x, y),(c, d) \in X^{2}$, temos:

$$
\begin{aligned}
A((x, y), \cdot)(c, d) & \doteq \frac{1}{2}[\gamma(x, d)+\gamma(y c)] \\
& =\frac{1}{2}[(x \gamma)(d)+(y \gamma)(c)] \\
& =\frac{1}{2}(y \gamma, x \gamma)(c, d) .
\end{aligned}
$$

Assim, para cada $(x, y) \in X^{2}$ :

$$
\begin{aligned}
\overline{(S, T)}(A)(x, y) & \doteq(S, T)(A((x, y), \cdot)) \\
& =(S, T)\left(\frac{1}{2} y \gamma, \frac{1}{2} x \gamma\right) \\
& =\frac{1}{2}[S(y \gamma)+T(x \gamma)] \\
& =\frac{1}{2}\left[S_{\gamma}(y)+T_{\gamma}(x)\right] \\
& =\frac{1}{2}\left(T_{\gamma}, S_{\gamma}\right)(x, y) .
\end{aligned}
$$

Pelo que acabamos de fazer, a extensão de $f$ é dada por:

$$
\bar{f}(S, T) \doteq \overline{(S, T)} \circ \overline{(S, T)}(A)
$$




$$
\begin{aligned}
& =\overline{(S, T)}\left(\frac{1}{2}\left(T_{\gamma}, S_{\gamma}\right)\right) \\
& =\frac{1}{2}\left[S\left(T_{\gamma}\right)+T\left(S_{\gamma}\right)\right] \\
& =\frac{1}{2}[S T+T S](\gamma) .
\end{aligned}
$$

Como visto acima, não é simples descrever a extensão de uma função holomorfa e este procedimento é feito apenas para algumas funções específicas. Mais exemplos podem ser encontrados em [Zal90].

A seguir apresentaremos uma aplicação do Teorema 7.2.1.

Definição 7.2.6. Seja $M$ um ELC.

(a) Uma rede $\left(x_{\alpha}\right)_{\alpha \in I} \subset M$ é dita de Cauchy se para cada vizinhança $U$ do zero, existe $\alpha_{0} \in I$ tal que $x_{\alpha}-x_{\beta} \in U$ para todos $\alpha, \beta \geq \alpha_{0}$.

(b) Um subconjunto $A \subset M$ é dito completo se cada rede de Cauchy em A converge a um ponto de $A$.

(c) $M$ é dito quase-completo se seus subconjuntos fechados e limitados são completos.

(d) Um subconjunto $A \subset M$ é dito limitante se:

$$
\|f\|_{A} \doteq \sup _{x \in A}|f(x)|<\infty \quad \text { para cada } f \in \mathscr{H}(M)
$$

Vamos assumir alguns resultados já que suas demonstrações fogem do escopo deste trabalho.

Proposição 7.2.7. Se A é um subconjunto fechado e limitante de um ELC separável quasecompleto, então A é compacto

Demonstração. Veja [Din12], Example 3.20(c).

O próximo resultado, provado por Dineen em [Din71], mostra que nem todo conjunto limitante e fechado de um ELC é compacto. Note que a base de Schauder $\left(e_{n}\right)$ de $c_{0}$ é um conjunto discreto e infinito, logo é fechado e não compacto.

Proposição 7.2.8. O conjunto $A=\left(e_{n}\right) \subset l_{\infty}$ é um limitante fechado e não compacto de $l_{\infty}$.

Demonstraçâo. Veja [Din12], Proposition 4.51.

Exemplo 7.2.9. Existe $f_{0} \in \mathscr{H}\left(c_{0}\right) \backslash \mathscr{H}_{b}\left(c_{0}\right)$ que não admite extensão holomorfa para $l_{\infty}$.

Considere $A=\left(e_{n}\right)$ a base de Schauder de $c_{0}$. Então A é fechado, não compacto e, pela Proposição 7.2.7, não é um subconjunto limitante de $c_{0}$. Existe $f_{0} \in \mathscr{H}\left(c_{0}\right)$ tal que $\left\|f_{0}\right\|_{A}=\infty$. Seja $\widetilde{f}_{0}$ uma extensão de $f_{0}$ para $l_{\infty}$ e suponha que $\widetilde{f}_{0}$ seja holomorfa. 
Como A é limitante de $l_{\infty}$, segue que $\left\|\widetilde{f}_{0}\right\|_{A}<\infty$. Por outro lado:

$$
\left\|\widetilde{f}_{0}\right\|_{A} \doteq \sup _{x \in A}\left|\widetilde{f}_{0}(x)\right|=\sup _{x \in A}\left|f_{0}(x)\right| \doteq\left\|f_{0}\right\|_{A}=\infty
$$

Logo, $f_{0}$ não admite extensão holomorfa para $l_{\infty}$.

Definição 7.2.10. Seja E um espaço de Banach. Uma rede $\left(x_{\alpha}\right) \subset E$ é dita w-Cauchy se, para cada $x^{\prime} \in E^{\prime}$, a rede $\left(x^{\prime}\left(x_{\alpha}\right)\right) \subset \mathbb{K}$ é de Cauchy.

Um subconjunto $A \subset E$ é dito fracamente condicionalmente compacto se toda sequência em $A$ admite subsequência w-Cauchy.

Em [Jos78], B. Josefson apresentou a seguinte caracterização dos subconjuntos limitantes de $l_{\infty}$ :

$A \subset l_{\infty}$ é limitante $\Longleftrightarrow A$ é fracamente condicionalmente compacto.

Proposição 7.2.11. Se $A \subset c_{0}$ é limitado, então $A$ é um subconjunto limitante de $l_{\infty}$.

Demonstração. Seja $r>0$ tal que $A \subset B(0, r)$. Então, pelo Teorema de Mazur:

$$
\bar{A}^{w} \subset \overline{B(0, r)}^{w}=\overline{B(0, r)}^{\|\cdot\|}=B[0, r]
$$

Logo $\bar{A}^{w}$ é limitado. Como $J_{c_{0}}\left(\bar{A}^{w}\right) \subset l_{\infty}$ é $w^{*}$-fechado e limitado, pelo Teorema de Alaoglu, é $w^{*}$-compacto. Pelo Teorema de Eberlein-Smulian, segue que $\bar{A}^{w}$ é fracamente sequencialmente compacto.

Dada uma sequência $\left(x_{n}\right) \subset A \subset \bar{A}^{w}$, existe uma subsequência $\left(x_{n_{k}}\right)$ fracamente convergente, isto é, para cada $x^{\prime} \in c_{0}^{\prime}$, a sequência $\left(x^{\prime}\left(x_{n_{k}}\right)\right) \subset \mathbb{K}$ é convergente. Logo $\left(x_{n}\right)$ é $w$-Cauchy e segue que $A$ é limitante de $l_{\infty}$.

Corolário 7.2.12. Uma função $f \in \mathscr{H}\left(c_{0}\right)$ admite extensão $\bar{f} \in \mathscr{H}\left(l_{\infty}\right)$ se, e somente se, $f$ é de tipo limitado.

Demonstração. Suponha que $f$ admita extensão $\bar{f} \in \mathscr{H}\left(l_{\infty}\right)$. Se $A \subset c_{0}$ é limitado, então $A$ é um subconjunto limitante de $l_{\infty}$ e temos:

$$
\|f\|_{A}=\|\bar{f}\|_{A}<\infty .
$$

Logo, $f$ é de tipo limitado. A recíproca segue do Teorema 7.2.1.

\subsection{Extensão de funções holomorfas arbitrárias}

Até agora, dada uma função $f \in \mathscr{H}_{b}(E, F)$, definimos uma extensão $\bar{f}$ estendendo suas diferenciais em 0 e utilizando a série de Taylor de $f$ em torno deste ponto. Por outro lado, se $f$ não é de 
tipo limitado seríamos forçados a definir $\bar{f}$ a partir de diferentes pontos do domínio de $f$. A seguir apresentaremos um método para estender uma função holomorfa arbitrária.

Dada $A \in \mathscr{L}\left({ }^{m} E, F\right)$, para cada $T \in G_{E F}$ e cada $k \in \mathbb{N}$, com $k \leq m$ denotaremos:

$$
\bar{T}^{k}(A) \doteq \underbrace{\bar{T} \circ \cdots \circ \bar{T}}_{k \text { vezes }}(A)
$$

Lema 7.3.1. Seja $f: E \longrightarrow F$ uma função holomorfa em $a \in E$. Então, para cada $p \in \mathbb{N}_{0}$ e cada $S \in G_{E F}$ com $\|S-a\|<\frac{R_{c} f(a)}{e}$, vale que:

$$
\sum_{k \geq p} \frac{\overline{S-a}^{(k-p)}}{(k-p) !}\left(D^{k} f(a)\right) \in \mathscr{L}^{s}\left({ }^{p} E, F\right)
$$

Demonstração. Seja $f(x)=\sum_{k=0}^{\infty} \frac{1}{k !} D^{k} f(a)(x-a)^{k}$ em torno de $a \in E$. Fixado $p \in \mathbb{N}_{0}$, observe que para cada $k \in \mathbb{N}_{0}, D^{k} f(a) \in \mathscr{L}^{s}\left({ }^{k} E, F\right)$. Logo, se $k \geq p$ :

$$
\overline{S-a}^{(k-p)}\left(D^{k} f(a)\right)=\underbrace{\overline{S-a} \circ \cdots \circ \overline{S-a}}_{k-p \text { vezes }}\left(D^{k} f(a)\right) \in \mathscr{L}^{s}\left({ }^{p} E, F\right) .
$$

Assim, basta verificar que a série é convergente.

$$
\sum_{k \geq p}\left\|\frac{\overline{S-a}^{(k-p)}}{(k-p) !}\left(D^{k} f(a)\right)\right\| \leq \sum_{k \geq p}\left\|\frac{1}{(k-p) !} D^{k} f(a)\right\|\|S-a\|^{k-p}
$$

Pelo Teorema de Cauchy-Hadamard 6.1.2, temos que $\limsup _{k}\left\|\widehat{\frac{D^{k} f(a)}{k !}}\right\|^{\frac{1}{k}}=\frac{1}{R_{c} f(a)}$. Além disso, aplicando a Desigualdade de Cauchy 6.2.6 segue que:

$$
\begin{aligned}
& \underset{k}{\limsup }\left\|\frac{1}{(k-p) !} D^{k} f(a)\right\|^{\frac{1}{k-p}}=\limsup _{k}\left(\frac{k !}{(k-p) !}\right)^{\frac{1}{k-p}}\left\|\frac{1}{k !} D^{k} f(a)\right\|^{\frac{1}{k-p}} \\
& =\limsup _{k}\left(\frac{k !}{(k-p) !}\right)^{\frac{1}{k-p}}\left(\left\|\frac{1}{k !} D^{k} f(a)\right\|^{\frac{1}{k}}\right)^{\frac{k}{k-p}} \\
& \leq \underset{k}{\limsup }\left(\frac{k !}{(k-p) !}\right)^{\frac{1}{k-p}}\left(\left(\frac{k^{k}}{k !}\left\|\frac{\widehat{D^{k} f(a)}}{k !}\right\|\right)^{\frac{1}{k}}\right)^{\frac{k}{k-p}} \\
& =\limsup _{k} \underbrace{\left(\frac{k !}{(k-p) !}\right)^{\frac{1}{k-p}}}_{(1)} \underbrace{\left(\frac{k^{k}}{k !}\right)^{\frac{1}{k-p}}}_{(2)} \underbrace{\left(\left\|\frac{D^{k} f(a)}{k !}\right\|^{\frac{1}{k}}\right)^{\frac{k}{k-p}}}_{(3)} .
\end{aligned}
$$


Em (1), fazendo a mudança $j=k-p$, o termo geral em (1) é dado por $x_{j} \doteq\left(\frac{(j+p) !}{j !}\right)^{\frac{1}{j}}$. Para ver que a sequência $\left(x_{j}\right)$ converge, vamos aplicar o teste da razão para $a_{j} \doteq \frac{(j+p) !}{j !}$. Temos:

$$
\frac{a_{j+1}}{a_{j}}=\frac{(j+p+1) !}{(j+1) !} \frac{j !}{(j+p) !}=\frac{j+p+1}{j+1} \stackrel{j \longrightarrow \infty}{\longrightarrow} 1 .
$$

Portando, $\limsup _{k}\left(\frac{k !}{(k-p) !}\right)^{\frac{1}{k-p}} \leq 1$.

Analogamente ao que acabamos de fazer em (1), segue que $\limsup _{k}\left(\frac{k^{k}}{k !}\right)^{\frac{1}{k-p}}=e$.

Vejamos o que ocorre em $(3)$. Dado $\varepsilon>0$, existe $j(\varepsilon) \in \mathbb{N}$ tal que $\left\|\frac{1}{j^{\prime}} \widehat{D^{j} f(a)}\right\|^{\frac{1}{j}}<\frac{1}{R_{c} f(a)}+\frac{\varepsilon}{2}$ sempre que $j \geq j(\varepsilon)$. Então:

$$
\underset{k}{\limsup }\left[\left\|\widehat{\frac{D^{k} f(a)}{k !}}\right\|^{\frac{1}{k}}\right]_{k}^{\frac{k}{k-p}} \leq \underset{k}{\limsup }\left(\frac{1}{R_{c} f(a)}+\frac{\varepsilon}{2}\right)^{\frac{k}{k-p}}=\frac{1}{R_{c} f(a)}+\frac{\varepsilon}{2}<\frac{1}{R_{c} f(a)}+\varepsilon
$$

Como $\varepsilon$ é arbitrário, segue que:

$$
\underset{k}{\limsup }\left[\left\|\widehat{\frac{D^{k} f(a)}{k !}}\right\|^{\frac{1}{k}} \leq \frac{1}{R_{c} f(a)}\right.
$$

Com as estimativas obtidas em (1), (2) e (3), temos:

$$
\left\|\frac{1}{(k-p) !} D^{k} f(a)\right\|^{\frac{1}{k-p}} \leq 1 \cdot e \cdot \frac{1}{R_{c} f(a)}=\frac{e}{R_{c} f(a)} .
$$

Logo, a série é absolutamente convergente sempre que $\|S-a\|<\frac{R_{c} f(a)}{e}$. Como $\mathscr{L}^{s}\left({ }^{p} E, F\right)$ é um espaço de Banach, segue que a série é convergente.

O resultado anterior nos permite definir localmente uma extensão de $f$ para $G_{E F}$ da seguinte maneira:

Definição 7.3.2. Seja $f: E \longrightarrow F$ holomorfa em $a \in$ E. Para $S \in G_{E F} \operatorname{com}\|S-a\|<\frac{R_{c} f(a)}{e}$, definimos:

$$
\bar{f}(S) \doteq \sum_{k \geq 0} \frac{1}{k !} \overline{D^{k} f(a)}(S-a, \ldots, S-a)=\sum_{k \geq 0} \frac{\overline{S-a}^{k}}{k !}\left(D^{k} f(a)\right)
$$

Observe que, se $f: E \longrightarrow F$ é holomorfa em $x, y \in E$ e $B\left(x, \frac{R_{c} f(x)}{e}\right) \cap B\left(y, \frac{R_{c} f(y)}{e}\right) \neq \emptyset$, devemos mostrar que as extensões locais de $f$, definidas em torno de $x$ e de $y$, coincidem. Para isso, precisamos do seguinte resultado: 
Lema 7.3.3. Seja $f: E \longrightarrow F$ uma função holomorfa em a $\in$ E. Então para cada $p \in \mathbb{N}_{0}, S \in G_{E F}$ com $\|S-a\|<\frac{R_{c} f(a)}{e}$ e $T \in G_{E F}$ tais que $\bar{S} \circ \bar{T}=\bar{T} \circ \bar{S}$, vale que:

$$
D^{p} \bar{f}(S)(T, \ldots, T)=\bar{T}^{p}\left(\sum_{k \geq p} \frac{\overline{S-a}^{(k-p)}}{(k-p) !}\left(D^{k} f(a)\right)\right)
$$

Demonstração. Faremos indução em $p \in \mathbb{N}$. Quando $p=0$, temos:

$$
D^{0} \bar{f}(S)(T)^{0}=\bar{f}(S)=\sum_{k \geq 0} \frac{\overline{S-a}^{k}}{k !}\left(D^{k} f(a)\right) .
$$

Agora suponha que o resultado seja válido para $p-1$. Segue da Observação 6.2.20 que:

$$
D^{p} \bar{f}(S)(T, \ldots, T)=\lim _{\lambda \rightarrow 0}\left[D^{p-1} \bar{f}(S+\lambda T)(T, \ldots, T)-D^{p-1} \bar{f}(S)(T, \ldots, T)\right]=(1)
$$

Como $\overline{S+\lambda T} \in B\left(a, \frac{R_{c} f(a)}{e}\right)$ para $|\lambda|$ suficientemente pequeno e $\overline{S+\lambda T}$ comuta com $\bar{T}$, segue da hipótese de indução que:

$$
\begin{aligned}
(1) & =\lim _{\lambda \rightarrow 0} \frac{1}{\lambda}\left[\bar{T}^{p-1}\left(\sum_{k \geq p-1} \frac{\overline{S+\lambda T-a}^{(k-p+1)}}{(k-p+1) !}\left(D^{k} f(a)\right)\right)-\bar{T}^{p-1}\left(\sum_{k \geq p-1} \frac{\overline{S-a}^{k-p+1}}{(k-p+1) !}\left(D^{k} f(a)\right)\right)\right] \\
& =\bar{T}^{p-1} \lim _{\lambda \rightarrow 0}\left[\frac { 1 } { \lambda } \left(\sum _ { k \geq p - 1 } \frac { 1 } { ( k - p + 1 ) ! } \left({\overline{S-a+\lambda T^{(k-p+1)}}}^{\left.\left.\left(k-p-\bar{S}^{(k-1)}\right)\right)\left(D^{k} f(a)\right)\right]=(2)}\right.\right.\right.
\end{aligned}
$$

Aplicando a Fórmula Binomial para $\left(\overline{S-a}+\lambda \bar{T}^{(k-p+1)}\right)$, como o primeiro termo do binômio e o termo em $k=p-1$ são nulos, obtemos:

$$
\begin{aligned}
(2) & =\bar{T}^{p-1} \lim _{\lambda \rightarrow 0} \frac{1}{\lambda}\left(\sum_{k \geq p} \frac{1}{(k-p+1) !} \sum_{i=1}^{k-p+1}\left(\begin{array}{c}
k-p+1 \\
i
\end{array}\right) \overline{S-a}^{(k-p+1-i)} \lambda^{i} \bar{T}^{i}\right)\left(D^{k} f(a)\right) \\
& =\bar{T}^{p-1} \lim _{\lambda \rightarrow 0} \sum_{k \geq p} \frac{1}{(k-p+1) !} \sum_{i=1}^{k-p+1}\left(\begin{array}{c}
k-p+1 \\
i
\end{array}\right) \overline{S-a}^{(k-p+1-i} \lambda^{i-1} \bar{T}^{i}\left(D^{k} f(a)\right) \\
& =\bar{T}^{p-1}\left(\sum_{k \geq p} \frac{(k-p+1)}{(k-p+1) !} \overline{S-a}^{(k-p)} \bar{T}\left(D^{k} f(a)\right)\right) \\
& =\bar{T}^{p}\left(\sum_{k \geq p} \frac{\overline{S-a}^{(k-p)}}{(k-p) !}\left(D^{k} f(a)\right)\right) .
\end{aligned}
$$

Observação 7.3.4. Se $S=z \in E$ e $T=y \in E$, temos:

$$
D^{p} f(z)(y, \ldots, y)=D^{p} \bar{f}(z)(y, \ldots, z)=\bar{y}^{p}\left(\sum_{k \geq p} \frac{\overline{z-a}(k-p)}{(k-p) !}\left(D^{k} f(a)\right)\right) .
$$


Isto é:

$$
D^{p} f(z)=\sum_{k \geq p} \frac{\overline{z-a}^{(k-p)}}{(k-p) !}\left(D^{k} f(a)\right) .
$$

Teorema 7.3.5. $\bar{f}$ está bem definida.

Demonstração. Suponha que $f: E \longrightarrow F$ seja holomorfa e que $x, y \in E$ são tais que $B\left(x, \frac{R_{c} f(x)}{e}\right) \cap$ $B\left(y, \frac{R_{c} f(y)}{e}\right) \neq \emptyset$ em $G_{E F}$. Denote as extensões locais de $f$ em $x$ e em $y$ por $F_{x}$ e $F_{y}$, respectivamente. Vejamos que $F_{x}$ e $F_{y}$ coincidem em um aberto de $G_{E F}$ contendo $x$ e $y$.

Seja $z \doteq \frac{R_{c} f(y) x+R_{c} f(x) y}{R_{c} f(x)+R_{c} f(y)} \in E \subset G_{E F}$ e seja $T \in B\left(x, \frac{R_{c} f(x)}{e}\right) \cap B\left(y, \frac{R_{c} f(y)}{e}\right)$. Segue que:

$$
\begin{aligned}
\|x-z\| & =\frac{1}{R_{c} f(x)+R_{c} f(y)}\left\|\left(R_{c} f(x)+R_{c} f(y)\right) x-R_{c} f(y) x-R_{c} f(x) y\right\| \\
& =\frac{R_{c} f(x)}{R_{c} f(x)+R_{c} f(y)}\|x-y\| \\
& \leq \frac{R_{c} f(x)}{R_{c} f(x)+R_{c} f(y)}(\|x-T\|+\|T-y\|) \\
& <\frac{R_{c} f(x)}{R_{c} f(x)+R_{c} f(y)}\left(\frac{R_{c} f(x)+R_{c} f(y)}{e}\right) \\
& =\frac{R_{c} f(x)}{e} .
\end{aligned}
$$

E, analogamente:

$$
\|y-z\|<\frac{R_{c} f(y)}{e}
$$

De (7.2) e de (7.3) segue que $z \in B\left(x, \frac{R_{c} f(x)}{e}\right) \cap B\left(y, \frac{R_{c} f(y)}{e}\right)$. Além disso, $\bar{z} \circ \bar{T}=\bar{T} \circ \bar{z}$ para todo $T \in G_{E F}$. Segue do Lema 7.3.3 que:

$$
\begin{aligned}
D^{p} F_{x}(z)(T, \ldots, T) & =\bar{T}^{p}\left(\sum_{k \geq p} \frac{\overline{z-x}^{(k-p)}}{(k-p) !}\left(D^{k} f(x)\right)\right) \\
& =\bar{T}^{p}\left(D^{p} f(z)\right) \\
& \doteq \overline{D^{p} f(z)}(T, \ldots, T) .
\end{aligned}
$$

E, analogamente:

$$
D^{p} F_{x}(z)(T, \ldots, T)=\overline{D^{p} f(z)}(T, \ldots, T)=D^{p} F_{y}(z)(T, \ldots, T) .
$$

Existe $\rho>0$ tal que $B(z, \rho) \subset B\left(x, \frac{R_{c} f(x)}{e}\right) \cap B\left(y, \frac{R_{c} f(y)}{e}\right)$. Segue do Teorema 6.1.5 que, para todo $S \in B(z, \rho)$, vale:

$$
F_{x}(S)=\sum_{k=0}^{\infty} \frac{1}{k !} D^{k} F_{x}(z)(S-z)^{k}
$$




$$
\begin{aligned}
& =\sum_{k=0}^{\infty} \frac{1}{k !} \overline{D^{k} f(z)}(S-z, \ldots, S-z) \\
& =\sum_{k=0}^{\infty} \frac{1}{k !} D^{k} F_{y}(z)(S-z)^{k} \\
& =F_{y}(S) .
\end{aligned}
$$

Logo, $\left.F_{x}\right|_{B(z, \rho)}=\left.F_{y}\right|_{B(z, \rho)}$ e, pelo Princípio da Identidade:

$$
\left.F_{x}\right|_{B\left(x, \frac{R_{c} f(x)}{e}\right) \cap B\left(y, \frac{R_{c} f(y)}{e}\right)}=\left.F_{y}\right|_{B\left(x, \frac{R_{c} f(x)}{e}\right) \cap B\left(y, \frac{R_{c} f(y)}{e}\right)} .
$$

E, portanto, $\bar{f}$ está bem definida.

Segue da demonstração anterior que se $f: U \subset E \longrightarrow F$ é holomorfa, podemos estender $f$ em cada ponto $x \in U$ e obter $\bar{f}$ definida em $\widetilde{U}=\bigcup_{x \in U} B\left(x, R_{c} \bar{f}(x)\right)$, onde $R_{c} \bar{f}(x) \geq \frac{R_{c} f(x)}{e}$.

Diferentes funções definidas num mesmo domínio podem ter raios de convergência distintos, logo $\widetilde{U}$ depende de $f$ e, por esta razão, não temos uma extensão $\bar{f}$ definida em $G_{E F}$ como na Seção 7.2. Isto é, as extensões de funções holomorfas têm diferentes domínios. Entretanto, em alguns casos é possível definir $\bar{f}$ em um domínio maior que $\widetilde{U}$. No artigo [Zal90] são apresentados alguns exemplos de tais funções.

\subsection{Caracterização da extensão de funções holomorfas}

Nesta seção apresentaremos uma caracterização da extensão de funções holomorfas em termos da continuidade do operador diferencial de primeira ordem, segundo [Za190].

Teorema 7.4.1. Sejam $g \in \mathscr{H}_{b}\left(E^{\prime \prime}\right)$ e $f \in \mathscr{H}_{b}(E)$ tais que $\left.g\right|_{E}=f$. São equivalentes:

(a) Para todo $x \in E, D g(x)$ é $w^{*}$-contínuo e para todos $z \in E^{\prime \prime}$ e $\left(x_{\alpha}\right) \subset E \operatorname{com} x_{\alpha} \stackrel{w^{*}}{\longrightarrow} z$, $D g(z)\left(x_{\alpha}\right) \longrightarrow D g(z)(z)$.

(b) $g=\bar{f}$.

Demonstração. $(a) \Longrightarrow(b)$ : Fixado $x \in E$, para cada $L \in E^{\prime \prime}$, escrevendo $g(z+\lambda L)$ e $g(z)$ em torno de $x$ e aplicando a Fórmula Binomial para $D^{k} g(x)([z-x]+\lambda L)^{k}$ em (1), obtemos:

$$
\begin{aligned}
D g(z)(L) & =\lim _{\lambda \rightarrow 0} \frac{1}{\lambda}[g(z+\lambda L)-g(z)] \\
& =\lim _{\lambda \rightarrow 0} \frac{1}{\lambda}\left[\sum_{k \geq 1} \frac{1}{k !}\left(D^{k} g(x)(z+\lambda L-x)^{k}-D^{k} g(x)(z-x)^{k}\right)\right] \\
& \stackrel{(1)}{=} \lim _{\lambda \rightarrow 0} \frac{1}{\lambda}\left[\sum_{k \geq 1} \frac{1}{k !} \sum_{i=1}^{k}\left(\begin{array}{c}
k \\
i
\end{array}\right) D^{k} g(x)(z-x)^{(k-i)}(\lambda L)^{i}\right]
\end{aligned}
$$




$$
\begin{aligned}
& =\lim _{\lambda \rightarrow 0} \sum_{k \geq 1} \frac{1}{k !} \sum_{i=1}^{k} D^{k} g(x)(z-x)^{(k-i)} \lambda^{(i-1)} L^{i} \\
& =\sum_{k \geq 1} \frac{1}{k !}\left(\begin{array}{c}
k \\
1
\end{array}\right) D^{k} g(x)(z-x)^{(k-1)} L^{1} \\
& =\sum_{k \geq 1} \frac{1}{(k-1) !} D^{k} g(x)(z-x, \ldots, z-x, L) .
\end{aligned}
$$

Logo, para todo $x \in E$, mostramos que para todo $z$ em torno de $x$ :

$$
D g(z)(L)=\sum_{k \geq 1} \frac{1}{(k-1) !} D^{k} g(x)(z-x, \ldots, z-x, L) .
$$

Dados $x \in E$ e $L \in E^{\prime \prime}$, pelo Teorema de Goldstine, existe uma rede $\left(x_{\alpha}\right) \subset E$ tal que $x_{\alpha} \stackrel{w^{*}}{\longrightarrow} L$ e $\left\|x_{\alpha}\right\| \leq\|L\|$. Vamos provar que para cada $n \in \mathbb{N}$, existe uma subrede $\left(x_{\alpha_{i}}\right)$ tal que, para cada $k \leq n$ :

$$
D^{k} g(x)\left(L, \ldots, L, x_{\alpha_{i}}\right) \longrightarrow D^{k} g(x)(L, \ldots, L)
$$

Provaremos (7.5) por indução em $n$. Quando $n=1$, como $D g(x)$ é $w^{*}$-convergente por hipótese, temos:

$$
D g(x)\left(x_{\alpha}\right) \longrightarrow D g(x)(L)
$$

Agora suponha que (7.5) seja válido para $(n-1)$. Então existe uma subrede $\left(x_{\alpha_{i}}\right)$ tal que:

$$
D^{k} g(x)\left(L, \ldots, L, x_{\alpha_{i}}\right) \longrightarrow D^{k} g(x)(L, \ldots, L) \text { para todo } k \leq n-1 .
$$

Note que, para cada $i \in \Lambda,\left\|x_{\alpha_{i}}\right\| \leq L$. Logo, o conjunto $\left\{D^{n} g(x)\left(L, \ldots, L, x_{\alpha_{i}}\right): i \in \Lambda\right\}$ é limitado em $\mathbb{C}$ e podemos escolher um ponto de acumulação $\tau_{n}$ e uma subrede $\left(x_{r}\right)$ de $\left(x_{\alpha_{i}}\right)$ tal que $D^{n} g(x)\left(L, \ldots, L, x_{r}\right) \longrightarrow \tau_{n}$.

Como $\left(x_{r}\right) \subset E$ e $x_{r} \stackrel{w^{*}}{\longrightarrow} L$, para cada $\lambda \in \mathbb{C},\left(x+\lambda x_{r}\right) \subset E$ é tal que $x+\lambda x_{r} \stackrel{w^{*}}{\longrightarrow} x+\lambda L$. Por hipótese, $D g(x+\lambda L)\left(x+\lambda x_{r}\right) \longrightarrow D g(x+\lambda L)(x+\lambda L)$ e pela linearidade de $D g(x+\lambda L)$ segue que $D g(x+\lambda L)\left(\lambda x_{r}\right) \longrightarrow D g(x+\lambda L)(\lambda L)$.

Então, por (7.4), temos:

$$
\begin{aligned}
D g(x & +\lambda L)\left(\lambda x_{r}\right)-D g(x+\lambda L)(\lambda L) \\
& =\sum_{k \geq 1} \frac{1}{(k-1) !}\left[D^{k} g(x)\left(\lambda L, \ldots, \lambda L, \lambda x_{r}\right)-D^{k} g(x)(\lambda L, \ldots, \lambda L, \lambda L)\right] \\
& =\sum_{k \geq 1} \frac{1}{(k-1) !} \lambda^{k}\left[D^{k} g(x)\left(L, \ldots, L, x_{r}\right)-D^{k} g(x)(L, \ldots, L)\right] .
\end{aligned}
$$

Tomando o limite em $r$, temos: 


$$
0=\left[\frac{\tau_{n}-D^{n} g(x)(L, \ldots, L)}{(n-1) !} \lambda^{n}+\lambda^{n+1} h_{n}(\lambda)\right]
$$

onde:

$$
h_{n}(\lambda)=\lim _{r}\left(\sum_{k \geq n+1} \frac{1}{(k-1) !} \lambda^{k-(n+1)}\left[D^{k} g(x)\left(L, \ldots, L, x_{r}\right)-D^{k} g(x)(L, \ldots, L)\right]\right) .
$$

Como, para todo $\lambda \in \mathbb{C} \backslash\{0\}$, temos que $D^{n} g(x)(L, \ldots, L)-\tau_{n}=\lambda(n-1) ! h_{n}(\lambda)$, se mostrarmos que $\left|h_{n}(\lambda)\right|$ é limitado em uma vizinhança do zero, teremos:

$$
D^{n} g(x)\left(L, \ldots, L, x_{r}\right) \longrightarrow \tau_{n}=D^{n} g(x)(L, \ldots, L)
$$

pois, se $\left|h_{n}(\lambda)\right| \leq M$ em uma vizinhança do zero, para $\lambda$ suficientemente pequeno:

$$
\left|D^{n} g(x)(L, \ldots, L)-\tau_{n}\right|=|\lambda|(n-1) !\left|h_{n}(\lambda)\right| \leq|\lambda|(n-1) ! M \stackrel{|\lambda| \rightarrow 0}{\longrightarrow} 0
$$

Vejamos que, de fato, $\left|h_{n}(\lambda)\right|$ é limitado em uma vizinhança do zero. Note que para todo $r$ e todo $k \in \mathbb{N}$, vale que:

$$
\begin{aligned}
\left|D^{k} g(x)\left(L, \ldots, L, x_{r}\right)-D^{k} g(x)(L, \ldots L)\right| & =\left|D^{k} g(x)\left(L, \ldots, L, x_{r}-L\right)\right| \\
& \leq\left\|D^{k} g(x)\right\|\|L\|^{k-1}\left\|x_{r}-L\right\| \\
& \leq 2\left\|D^{k} g(x)\right\|\|L\|^{k} .
\end{aligned}
$$

Então:

$$
\begin{aligned}
\left|h_{n}(\lambda)\right| & \leq \sum_{k \geq n+1} \frac{2\left\|D^{k} g(x)\right\|}{(k-1) !}\|L\|^{k}|\lambda|^{k-(n+1)} \\
& =\sum_{j \geq 0} \frac{2\left\|D^{n+1+j} g(x)\right\|}{(n+j) !}\|L\|^{n+1+j}|\lambda|^{j} \\
& =2\|L\|^{n+1} \sum_{j \geq 0} \frac{\left\|D^{n+1+j} g(x)\right\|}{(n+j) !}\|L\|^{j}|\lambda|^{j} .
\end{aligned}
$$

Faremos um argumento análogo ao que foi feito no Lema 7.3.1 para mostrar que $\left|h_{n}(\lambda)\right|$ é limitado em torno do zero. Pelo Critério de Cauchy-Hadamard, $\underset{k}{\limsup }\left\|\frac{1}{k !} \widehat{D^{k} g(x)}\right\|^{\frac{1}{k !}}=\frac{1}{R_{c} g(x)}$.

Além disso, aplicando a Desigualdade de Cauchy, segue que:

$$
\limsup _{j}\left(\frac{\left\|D^{n+1+j} g(x)\right\|}{(n+j) !}\right)^{\frac{1}{j}}=\limsup (n+j+1)^{\frac{1}{j}}\left\|\frac{D^{n+j+1} g(x)}{(n+j+1) !}\right\|^{\frac{1}{j}}
$$




$$
\begin{aligned}
& \leq \limsup _{j}(n+j+1)^{\frac{1}{j}}\left(\frac{(n+j+1)^{n+j+1}}{(n+j+1) !}\right)^{\frac{1}{j}}\left\|\frac{D^{n \widehat{+j+1} g}(x)}{(n+j+1) !}\right\|^{\frac{1}{j}} \\
& =\limsup _{j} \underbrace{(n+j+1)^{\frac{1}{j}}}_{(i)} \underbrace{\left(\frac{(n+j+1)^{n+j+1}}{(n+j+1) !}\right)^{\frac{1}{j}}}_{(i i)}(\underbrace{\left.\left\|\frac{D^{n+j+1} g}{(n+j)}\right\|^{\frac{1}{n+j+1}}\right)^{\frac{n+j+1}{j}}}_{(i i i)})^{\frac{n+1) !}{n+j+1)}}
\end{aligned}
$$

Em $(i)$, utilizando o critério da razão para a sequência $a_{j} \doteq n+j+1$, segue que:

$$
\limsup _{j}\left(a_{j}\right)^{\frac{1}{j}} \leq \limsup _{j} \frac{a_{j+1}}{a_{j}}=\lim _{j} \frac{a_{j+1}}{a_{j}}=1 .
$$

Procedendo da mesma maneira em $(i i)$, com a sequência $b_{j} \doteq \frac{(n+j+1)^{n+j+1}}{(n+j+1) !}$, segue:

$$
\limsup _{j}\left(b_{j}\right)^{\frac{1}{j}} \leq \limsup _{j} \frac{b_{j+1}}{b_{j}}=\lim _{j} \frac{b_{j+1}}{b_{j}}=e .
$$

Em $($ iii $)$, note que para cada $\varepsilon>0$, existe $j(\varepsilon) \in \mathbb{N}$ tal que $\left\|\frac{D^{n+j+1} g(x)}{(n+j+1) !}\right\|^{\frac{1}{n+j+1}}<\frac{1}{R_{c} g(x)}+\frac{\varepsilon}{2}$, sempre que $j \geq j(\varepsilon)$. Logo:

$$
\begin{aligned}
\limsup _{j}\left(\left\|\frac{D^{n+j+1} g(x)}{(n+j+1) !}\right\|^{\frac{1}{n+j+1}}\right)^{\frac{n+j+1}{j}} & \leq \limsup _{j}\left[\left(\frac{1}{R_{c} g(x)}+\frac{\varepsilon}{2}\right)^{n+j+1}\right]^{\frac{1}{j}} \\
& \leq \frac{1}{R_{c} g(x)}+\frac{\varepsilon}{2} \\
& <\frac{1}{R_{c} g(x)}+\varepsilon .
\end{aligned}
$$

Como $\varepsilon$ é arbitrário, segue que:

$$
\underset{j}{\limsup }\left(\left\|\frac{D^{n+j+1} g(x)}{(n+j+1) !}\right\|^{\frac{1}{n+j+1}}\right)^{\frac{n+j+1}{j}} \leq \frac{1}{R_{c} g(x)} .
$$

Consequentemente:

$$
\limsup _{j}\left(\frac{\left\|D^{n+1+j} g(x)\right\|}{(n+j) !}\right)^{\frac{1}{j}} \leq \frac{e}{R_{c} g(x)} .
$$

Logo, se $|\lambda|<\frac{R_{c} g(x)}{e\|L\|}$, a série é convergente. Portanto, $\left|h_{n}(\lambda)\right| \leq M$ em uma vizinhança do zero e (7.5) está provado.

Agora provaremos que, para todos $x \in E, L \in E^{\prime \prime}$ e $k \in \mathbb{N}, D^{k} g(x)(L, \ldots, L)=\overline{D^{k} f(x)}(L, \ldots, L)$. Provaremos por indução em $k$.

Quando $k=1$, note que $D g(x)$ é $w^{*}$-contínua por hipótese e $\overline{D f(x)}$ é $w^{*}$-contínua pela sua definição. Se $L \in E^{\prime \prime}$, pelo Teorema de Goldstine, existe uma rede $\left(x_{\alpha}\right) \subset E$ tal que $x_{\alpha} \stackrel{w^{*}}{\longrightarrow} L$. 
Segue que $D g(x)\left(x_{\alpha}\right) \stackrel{w^{*}}{\longrightarrow} D g(x)(L)$ e, como $\left.g\right|_{E}=f$, temos:

$$
D g(x)\left(x_{\alpha}\right)=D f(x)\left(x_{\alpha}\right)=\overline{D f(x)}\left(x_{\alpha}\right) \stackrel{w^{*}}{\longrightarrow} \overline{D f(x)}(L)
$$

Portanto, $D g(x)=\overline{D f(x)}$ em $E^{\prime \prime}$. Agora suponha que o resultado valha para $k-1$. Dados $L \in E^{\prime \prime}$ e $\left(x_{\alpha}\right) \subset E$ tais que $x_{\alpha} \stackrel{w^{*}}{\longrightarrow} L$, pela hipótese de indução, temos:

$$
\begin{aligned}
& D^{k} g(x)\left(L, \ldots, L, x_{\alpha}\right)=\lim _{\lambda \rightarrow 0} \frac{1}{\lambda}\left[D^{k-1} g\left(x+\lambda x_{\alpha}\right)(L, \ldots, L)-D^{k-1} g(x)(L, \ldots, L)\right] \\
& =\lim _{\lambda \rightarrow 0} \frac{1}{\lambda}\left[\overline{D^{k-1} f\left(x+\lambda x_{\alpha}\right)}(L, \ldots, L)-\overline{D^{k-1} f(x)}(L, \ldots, L)\right] \\
& =\bar{L}^{k-1}\left(\lim _{\lambda \rightarrow 0} \frac{1}{\lambda}\left[D^{k-1} f\left(x+\lambda x_{\alpha}\right)-D^{k-1} f(x)\right]\right) \\
& =\bar{L}^{k-1}(\underbrace{D^{k} f(x)\left(x_{\alpha}\right)}_{\in \mathscr{L}^{s}(k-1 E)}) \\
& =\left[\bar{L}^{k-1}\left(D^{k} f(x)\right)\right]\left(x_{\alpha}\right) \\
& =\overline{x_{\alpha}} \circ \bar{L}^{k-1}\left(D^{k} f(x)\right) \text {. }
\end{aligned}
$$

Agora, tomando o limite em uma subrede adequada $\left(x_{r}\right)$ de $\left(x_{\alpha}\right)$, por $(7.5)$ :

$$
D^{k} g(x)(L, \ldots, L)=\bar{L}^{k}\left(D^{k} f(x)\right) \doteq \overline{D^{k} f(x)}(L, \ldots, L)
$$

Para mostrar que $\bar{f}=g$, sejam $x \in E$ e $L \in E^{\prime \prime}$. Então, em torno de $x$ :

$$
g(x+L)=\sum_{k \geq 0} \frac{1}{k !} D^{k} g(x)(L, \ldots, L)=\sum_{k \geq 0} \frac{1}{k !} \overline{D^{k} f(x)}(L, \ldots, L)=\bar{f}(x+L) .
$$

$(b) \Longrightarrow(a):$ Dados $z, T \in E^{\prime \prime}$, temos:

$$
\begin{aligned}
D \bar{f}(z)(T) & =\lim _{\lambda \rightarrow 0} \frac{1}{\lambda}[\bar{f}(z+\lambda T)-\bar{f}(z)] \\
& =\lim _{\lambda \rightarrow 0} \frac{1}{\lambda}\left[\sum_{k \geq 1} \frac{1}{k !}\left(\overline{D^{k} f(0)}(z+\lambda T)^{k}-\overline{D^{k} f(0)} z^{k}\right)\right]=(*)
\end{aligned}
$$

Note que $\overline{D^{k} f(0)}$ não é necessariamente simétrica e como $\bar{z}$ e $\overline{\lambda T}$ não comutam, não podemos utilizar a Fórmula Binomial como antes. Entretanto, como $\overline{D^{k} f(0)}$ é multilinear, podemos desenvolver $\overline{D^{k} f(0)}(z+\lambda T)^{k}$ em uma soma finita e reordenar os termos da seguinte maneira:

$$
\overline{D^{k} f(0)}(z+\lambda T)^{k}=S_{0}+\lambda S_{1}+\lambda^{2} S_{2}+\cdots+\lambda_{k-1} S_{k-1}+\lambda^{k} S_{k},
$$

onde, para cada $0 \leq j \leq k, S_{j}$ é a soma dos termos em que $\overline{D^{k} f(0)}$ tem exatamente $j$ entradas 
iguais a $\lambda T$. Desta maneira, temos:

$$
\begin{aligned}
S_{0} & =\overline{D^{k} f(0)}(z, \ldots, z) \\
S_{1} & =\overline{D^{k} f(0)}(z, \ldots, z, T)+\overline{D^{k} f(0)}(z, \ldots, z, T, z)+\cdots+\overline{D^{k} f(0)}(T, z, \ldots, z) \\
& \vdots \\
S_{k} & =\lambda^{k} \overline{D^{k} f(0)}(T, \ldots, T)
\end{aligned}
$$

Logo, temos que:

$$
\begin{aligned}
(*)=\lim _{\lambda \rightarrow 0} \frac{1}{\lambda}\left[\sum_{k \geq 1} \frac{1}{k !}\left(\lambda S_{1}+\ldots \lambda^{k} S_{k}\right)\right] & =\lim _{\lambda \rightarrow 0} \sum_{k \geq 1} \frac{1}{k !}\left(S_{1}+\lambda S_{2}+\cdots+\lambda^{k-1} S_{k}\right) \\
& =\sum_{k \geq 1} \frac{1}{k !}\left(\overline{D^{k} f(0)}(z, \ldots, z, T)+\cdots+\overline{D^{k} f(0)}(T, z, \ldots, z)\right) .
\end{aligned}
$$

Agora suponha que $z \in E$ e que $\left(T_{\alpha}\right) \subset E^{\prime \prime}$ seja uma rede tal que $T_{\alpha} \stackrel{w^{*}}{\longrightarrow} T$. Então:

$$
\overline{D^{k} f(0)}\left(z, \ldots, z, T_{\alpha}, z, \ldots, z\right)=\overline{D^{k} f(0)}\left(T_{\alpha}, z, \ldots, z\right) \longrightarrow \overline{D^{k} f(0)}(T, z, \ldots, z)
$$

E temos que:

$$
D \bar{f}(z)\left(T_{\alpha}\right)=\sum_{k \geq 1} \frac{1}{(k-1) !} \overline{D^{k} f(0)}\left(T_{\alpha}, z, \ldots, z\right) .
$$

Dado $\varepsilon>0$, seja $j_{0} \in \mathbb{N}$ tal que $\sum_{k=j}^{l} \frac{1}{(k-1) !}<\sqrt{\varepsilon}$ sempre que $l>j \geq j_{0}$.

Dados $l>j \in \mathbb{N}$, como $\overline{D^{k} f(0)}\left(T_{\alpha}, z, \ldots, z\right) \longrightarrow \overline{D^{k} f(0)}(T, z, \ldots, z)$ para todo $k \in \mathbb{N}$, existe $\alpha_{0} \in \Lambda$ tal que, para todo $j \leq k \leq l$ :

$$
\alpha \geq \alpha_{0} \Longrightarrow\left|\overline{D^{k} f(0)}\left(T_{\alpha}-T, z, \ldots, z\right)\right|<\sqrt{\varepsilon}
$$

Então, para todos $l>j \geq j_{0}$ e $\alpha \geq \alpha_{0}$, temos:

$$
\begin{aligned}
& \left|\sum_{k=j}^{l} \frac{1}{(k-1) !}\left[\overline{D^{k} f(0)}\left(T_{\alpha}, z, \ldots, z\right)-\overline{D^{k} f(0)}(T, z, \ldots, z)\right]\right| \\
& \quad=\left|\sum_{k=j}^{l} \frac{1}{(k-1) !}\left[\overline{D^{k} f(0)}\left(T_{\alpha}-T, z, \ldots, z\right)\right]\right| \\
& \quad \leq \sum_{k=j}^{l} \frac{1}{(k-1) !}\left|\overline{D^{k} f(0)}\left(T_{\alpha}-T, z, \ldots, z\right)\right| \\
& \quad<\varepsilon
\end{aligned}
$$


Acabamos de provar que:

$$
D \bar{f}(z)\left(T_{\alpha}\right)=\sum_{k \geq 1} \frac{1}{(k-1) !} \overline{D^{k} f(0)}\left(T_{\alpha}, z, \ldots, z\right) \longrightarrow \sum_{k \geq 1} \frac{1}{(k-1) !} \overline{D^{k} f(0)}(T, z, \ldots, z)=D \bar{f}(z)(T)
$$

que é a primeira parte de $(a)$.

Agora suponha que $z \in E^{\prime \prime}$ e que $\left(x_{\alpha}\right) \subset E$ seja tal que $x_{\alpha} \stackrel{w^{*}}{\longrightarrow} z$. Então:

$$
\overline{D^{k} f(0)}\left(z, \ldots, z, x_{\alpha}, z, \ldots, z\right)=\overline{D^{k} f(0)}\left(x_{\alpha}, z, \ldots, z\right)
$$

E temos que:

$$
D \bar{f}(z)\left(x_{\alpha}\right)=\sum_{k \geq 1} \frac{1}{(k-1) !} \overline{D^{k} f(0)}\left(x_{\alpha}, z, \ldots, z\right)
$$

E, com argumento análogo, esta série converge para $\sum_{k \geq 1} \frac{1}{(k-1) !} \overline{D^{k} f(0)}(z, \ldots, z)=D \bar{f}(z)(z)$. Segue que:

$$
D g(z)\left(x_{\alpha}\right)=D \bar{f}(z)\left(x_{\alpha}\right) \longrightarrow D \bar{f}(z)(z)=D g(z)(z)
$$

Os próximos exemplos esclarecem a primeira condição do Teorema anterior.

Exemplo 7.4.2. Se $z \in E^{\prime \prime} \backslash E$, então $\bar{D} \bar{f}(z)$ não é necessariamente $w^{*}$-contínuo.

Seja X uma álgebra de Banach comutativa tal que o produto de Arens não é comutativo. Pelo Teorema 5.3.1, o produto de Arens em $X^{\prime \prime}$ não é $w^{*}$-contínuo na segunda entrada.

Tome $T, S \in X^{\prime \prime}$ e uma rede $\left(S_{i}\right) \subset X^{\prime \prime}, S_{i} \stackrel{w^{*}}{\longrightarrow} S$, tal que $T S_{i} \stackrel{w^{*}}{\longrightarrow} T S$. Então existe $\gamma \in X^{\prime}$ tal que $T S_{i}(\gamma) \nrightarrow T S(\gamma)$. Considere a função $f: X^{2} \longrightarrow \mathbb{C}$ dada por $f(a, b) \doteq \gamma(a b)$.

Então $z_{i}=\left(S_{i}, T\right) \stackrel{w^{*}}{\longrightarrow} z=(S, T)$. A função $\bar{f}$ é bilinear e, pelo Exemplo 7.2.5, temos:

$$
\begin{aligned}
D \bar{f}(z)\left(z_{i}\right)-D \bar{f}(z)(z) & =D \bar{f}(S, T)\left(S_{i}, T\right)-D \bar{f}(S, T)(S, T) \\
& =\bar{f}(S, T)+\bar{f}\left(S_{i}, T\right)-\bar{f}(S, T)-\bar{f}(S, T) \\
& =\frac{1}{2}\left[S T+T S+S_{i} T+T S_{i}-S T-T S-S T-T S\right](\gamma) \\
& =\frac{1}{2}\left[\left(S_{i} T-S T\right)+\left(T S_{i}-T S\right)\right](\gamma) .
\end{aligned}
$$

Note que $S_{i} T-S T \stackrel{w^{*}}{\longrightarrow} 0$. Assim $D \bar{f}(z)\left(z_{i}\right) \stackrel{w^{*}}{\longrightarrow} D \bar{f}(z)(z)$ se, e somente se, $T S_{i} \stackrel{w^{*}}{\longrightarrow} T S$, o que não ocorre. Logo $D \bar{f}(z)$ não é $w^{*}$-contínuo.

Exemplo 7.4.3. A continuidade $w^{*}$ de $D g(x)$ para todo $x \in E$ não é suficiente para garantir $g=\bar{f}$ onde $f=\left.g\right|_{E}$.

Sejam $X$ uma álgebra de Banach comutativa, $\gamma \in X^{\prime}, f: X^{2} \longrightarrow \mathbb{C}$ dada por $f(a, b) \doteq \gamma(a b) e$ $g:\left(X^{\prime \prime}\right)^{2} \longrightarrow \mathbb{C}$ dada por $g(S, T)=(S T)(\gamma)$. 
Então $\left.g\right|_{X}=f, g$ é bilinear e, se $(a, b) \in X^{2}$ e $(U, V) \in\left(X^{\prime \prime}\right)^{2}$, temos:

$$
D g(a, b)(U, V)=g(a, V)+g(U, b)=(a V+U b)(\gamma)
$$

que é $w^{*}$-contínua em $(U, V)$. Entretanto, segue do Exemplo 7.2 .5 que $g \neq \bar{f}$.

Antes de enunciar as consequências do Teorema 7.4.1, introduziremos algumas notações para simplificar a escrita.

Dados $n, k \in \mathbb{N}$, denotaremos por $\mathscr{H}_{b}\left(\mathbb{C}^{n}\right)^{k} \doteq \underbrace{\mathscr{H}_{b}\left(\mathbb{C}^{n}\right) \times \cdots \times \mathscr{H}_{b}\left(\mathbb{C}^{n}\right)}_{k \text { vezes }}$.

Assim, cada $G \in \mathscr{H}_{b}\left(\mathbb{C}^{n}\right)^{k}$ se escreve como $G=\left(G_{1}, \ldots, G_{k}\right)$, onde $G_{i}: \mathbb{C}^{n} \longrightarrow \mathbb{C}$ é holomorfa de tipo limitado para cada $1 \leq i \leq k$.

Dada $\left(f_{1}, \ldots, f_{n}\right) \in \mathscr{H}_{b}(E)^{n}$, definimos a função $G\left(f_{1}, \ldots, f_{n}\right): E \longrightarrow \mathbb{C}^{k}$ da seguinte maneira:

$$
G\left(f_{1}, \ldots, f_{n}\right)(x) \doteq\left(G_{1}\left(f_{1}(x), \ldots, f_{n}(x)\right), \ldots, G_{k}\left(f_{1}(x), \ldots, f_{n}(x)\right)\right), \quad \text { para cada } x \in E
$$

Corolário 7.4.4. Se $f_{1}, \ldots, f_{n} \in \mathscr{H}_{b}(E)$ e $G \in \mathscr{H}_{b}\left(\mathbb{C}^{n}\right)^{k}$ é tal que $G\left(f_{1}, \ldots, f_{n}\right)=0$, então $G\left(\overline{f_{1}}, \ldots, \overline{f_{n}}\right)=0$.

Demonstração. Denote $G=\left(G_{1}, \ldots, G_{k}\right)$ e defina, para cada $1 \leq i \leq k$, a função $F_{i}: E^{\prime \prime} \longrightarrow \mathbb{C}$ por $F_{i}(T) \doteq G_{i}\left(\overline{f_{1}}(T), \ldots, \overline{f_{n}}(T)\right)$.

Assim $F_{i}$ é composta de funções holomorfas e segue que $F_{i} \in \mathscr{H}_{b}\left(E^{\prime \prime}\right)$. Além disso:

$$
\left.F_{i}\right|_{E}=\left.\pi_{i} \circ G\left(\overline{f_{1}}, \ldots, \overline{f_{n}}\right)\right|_{E}=\pi_{i} \circ G\left(f_{1}, \ldots, f_{n}\right)=0 .
$$

Para cada $z \in E^{\prime \prime}$, temos:

$$
\begin{aligned}
D F_{i}(z) & =D G_{i}\left(\overline{f_{1}}(z), \ldots, \overline{f_{n}}(z)\right) \circ\left(D \overline{f_{1}}, \ldots, D \overline{f_{n}}\right)(z) \\
& =D G_{i}\left(\overline{f_{1}}(z), \ldots, \overline{f_{n}}(z)\right) \circ\left(D \overline{f_{1}}(z), \ldots, D \overline{f_{n}}(z)\right) .
\end{aligned}
$$

Como $\mathbb{C}^{n}$ é reflexivo, $G_{i}=\overline{G_{i}}$ satisfaz a condição (a) do Teorema 7.4.1. Segue que $F_{i}$ é extensão da função nula para $E^{\prime \prime}$ e, portanto, $F_{i}=0$ para todo $1 \leq i \leq k$.

Corolário 7.4.5. A aplicação $\Psi: \mathscr{H}_{b}(E) \longrightarrow \mathscr{H}_{b}\left(E^{\prime \prime}\right)$ dada por $\Psi(f)=\bar{f}$ é um morfismo de álgebras.

Demonstração. Já vimos que $\Psi$ é contínua e linear. Resta verificar que é multiplicativa. Considere a aplicação $G: \mathbb{C}^{3} \longrightarrow \mathbb{C}$ dada por $G(u, v, w) \doteq w-u v$. 
Dadas $f, g \in \mathscr{H}_{b}(E)$, temos:

$$
G(f, g, f g)(x)=G(f(x), g(x), f(x) g(x))=f(x) g(x)-f(x) g(x)=0 .
$$

Então, para cada $z \in E^{\prime \prime}$, temos:

$$
0=G(\bar{f}(z), \bar{g}(z), \overline{f g}(z))=\overline{f g}(z)-\bar{f}(z) \bar{g}(z) .
$$

Logo, $\overline{f g}=\bar{f} \bar{g}$. 


\section{Referências Bibliográficas}

[AB78] Richard M Aron e Paul D Berner. A Hahn-Banach extension theorem for analytic mappings. Bulletin de la Société Mathématique de France, 106:3-24, 1978. 1, 40, 45, 86

[ABC01] Richard Aron, Christopher Boyd e Yun Sung Choi. Unique Hahn-Banach theorems for spaces of homogeneous polynomials. Journal of the Australian Mathematical Society, $70(03): 387-400,2001$. 1

[AGM03] Richard M Aron, Domingo García e Manuel Maestre. On norm attaining polynomials. Publications of the Research Institute for Mathematical Sciences, 39(1):165-172, 2003. 36

[Are51a] Richard Arens. The adjoint of a bilinear operation. Proceedings of the American Mathematical Society, 2(6):839-848, 1951. 31

[Are51b] Richard Arens. Operations induced in function classes. Monatshefte für Mathematik, $55(1): 1-19,1951.31$

[Cha85] Soo Bong Chae. Holomorphy and Calculus in Normed Spaces, volume 92. CRC Press, 1985. $2,9,69,73$

[Dan13] Sheldon Miriel Gil Dantas. A evolução do estudo das aplicações lineares e não lineares que atingem a norma em espaços de Banach. Dissertação de Mestrado, Universidade de São Paulo, 2013. 2, 29

[DG89] Alexander M Davie e Theodore W Gamelin. A theorem on polynomial-star approximation. Proceedings of the American Mathematical Society, 106(2):351-356, 1989. 1, $2,46,48,86$

[DH79] John Duncan e Seyed Ali-Reza Hosseiniun. The second dual of a Banach algebra. Proceedings of the Royal Society of Edinburgh: Section A Mathematics, 84(3-4):309325, 1979. 63

[Din71] Seán Dineen. Bounding subsets of a Banach space. Mathematische Annalen, 192(1):6170, 1971. 88

[Din12] Seán Dineen. Complex Analysis on Infinite Dimensional spaces. Springer Science \& Business Media, 2012. 2, 40, 88

[GGM93] Pablo Galindo, Domingo García e Manuel Maestre. Entire functions of bounded type on Fréchet spaces. Mathematische Nachrichten, 161(1):185-198, 1993. 1

[GGMM94] Pablo Galindo, Domingo García, Manuel Maestre e Jorge Mujica. Extension of multilinear mappings on Banach spaces. Studia Mathematica, 108(1):55-76, 1994. 1

[GI88] Gilles Godefroy e Bruno Iochum. Arens-regularity of Banach algebras and the geometry of Banach spaces. Journal of Functional Analysis, 80(1):47-59, 1988. 63, 65 
[Her06] Israel N Herstein. Topics in Algebra. John Wiley \& Sons, 2006. 14

[IL89] Bruno Iochum e Guy Loupias. Arens regularity and local reflexivity principle for Banach algebras. Mathematische Annalen, 284(1):23-40, 1989. 63

[Jos78] Bengt Josefson. Bounding subsets of $l^{\infty}(A)$. Journal de Mathématiques Pures et Appliquées, 57(4):397-421, 1978. 89

[Leu96] Denny H Leung. Some remarks on regular Banach spaces. Glasgow Mathematical Journal, 38(02):243-248, 1996. 65

[LZ00] Silvia Lassalle e Ignacio Zalduendo. To what extent does the dual Banach space $E^{\prime}$ determine the polynomials over E? Arkiv för Matematik, 38(2):343-354, 2000. 1

[Meg12] Robert E Megginson. An introduction to Banach Space Theory, volume 183 of Graduate Texts in Mathematics. Springer Science \& Business Media, 2012. 1, 2, 3, 53, 59

[Muj86] Jorge Mujica. Complex Analysis in Banach Spaces. North-Holland, 1986. 2, 9, 69, 73, $74,75,76,77$

[MV97] Reinhold Meise e Dietmar Vogt. Introduction to Functional Analysis. Clarendon Press, 1997. 76

[Nac69] Leopoldo Nachbin. Topology on Spaces of Holomorphic Mappings. Springer-Verlag New York Inc., PWN-Polish Scientific Publishers, 1969. 2, 69, 73

[Ren66] John F Rennison. A note on the extension of associative products. Proceedings of the American Mathematical Society, 17(6):1375-1377, 1966. 61

[SGD00] Félix Cabello Sánchez, Ricardo García González e Ignacio Villanueva Díez. Extension of multilinear operators on Banach spaces. Extracta mathematicae, 15(2):291-334, 2000. 1

[Za190] Ignacio Zalduendo. A canonical extension for analytic functions on Banach spaces. Transactions of the American Mathematical Society, 320(2):747-763, 1990. 1, 2, 79, 88,94

[Zal91] Ignacio Zalduendo. A simple example of a non-commutative Arens product. Publicacions Matemàtiques, 35(2):475-477, 1991. 61

[Za105] Ignacio Zalduendo. Extending polynomials on Banach spaces - a survey. Revista de la Unión Matemática Argentina, 46(2):45-72, 2005. 1, 2, 61, 63 